\title{
Search for the Production of a Long-Lived Neutral Particle Decaying within the ATLAS Hadronic Calorimeter in Association with a $Z$ Boson from pp Collisions at $\sqrt{s}=13 \mathrm{TeV}$
}

\author{
M. Aaboud et al." \\ (ATLAS Collaboration)
}

(Received 7 November 2018; published 15 April 2019)

\begin{abstract}
This Letter presents a search for the production of a long-lived neutral particle $\left(Z_{d}\right)$ decaying within the ATLAS hadronic calorimeter, in association with a standard model (SM) $Z$ boson produced via an intermediate scalar boson, where $Z \rightarrow \ell^{+} \ell^{-}(\ell=e, \mu)$. The data used were collected by the ATLAS detector during 2015 and $2016 p p$ collisions with a center-of-mass energy of $\sqrt{s}=13 \mathrm{TeV}$ at the Large Hadron Collider and correspond to an integrated luminosity of $36.1 \pm 0.8 \mathrm{fb}^{-1}$. No significant excess of events is observed above the expected background. Limits on the production cross section of the scalar boson times its decay branching fraction into the long-lived neutral particle are derived as a function of the mass of the intermediate scalar boson, the mass of the long-lived neutral particle, and its $c \tau$ from a few centimeters to one hundred meters. In the case that the intermediate scalar boson is the SM Higgs boson, its decay branching fraction to a long-lived neutral particle with a $c \tau$ approximately between 0.1 and $7 \mathrm{~m}$ is excluded with a $95 \%$ confidence level up to $10 \%$ for $m_{Z_{d}}$ between 5 and $15 \mathrm{GeV}$.
\end{abstract}

DOI: 10.1103/PhysRevLett.122.151801

Many extensions to the standard model (SM) such as supersymmetry [1,2], inelastic dark matter [3], and hidden valley scenarios $[4,5]$ predict the existence of long-lived neutral particles that can decay hadronically. Search for long-lived neutral particles is an emerging field of research that has attracted significant theoretical and experimental interests. So far, only searches for the pair production of such particles have been carried out by the ATLAS [6-9], CMS [10,11], and LHCb [12,13] experiments at the Large Hadron Collider (LHC), and the CDF [14], and D0 [15] experiments at the Tevatron.

This Letter reports a new way to look for new physics (NP) beyond the SM in a collider using singly produced long-lived neutral particle, which is one potential scenario that NP can manifest itself but had never been considered in theories or experiments. Among many possible single production final states, this Letter focuses on search for a hadronically decaying long-lived neutral particle, denoted by $Z_{d}$ hereafter, produced in association with a SM $Z$ boson through an intermediate scalar $\Phi$ or Higgs boson, $p p \rightarrow \Phi / H \rightarrow Z Z_{d}$, where $Z \rightarrow \ell^{+} \ell^{-}(\ell=e, \mu)$. Production of a new particle in association with a $Z$ boson is a popular scenario in hidden- or dark-sector models with

\footnotetext{
*Full author list given at the end of the article.

Published by the American Physical Society under the terms of the Creative Commons Attribution 4.0 International license. Further distribution of this work must maintain attribution to the author(s) and the published article's title, journal citation, and DOI. Funded by SCOAP ${ }^{3}$.
}

an additional $U(1)_{d}$ dark gauge symmetry [16,17]. One such model has been tested by the ATLAS experiment in a search for a new particle that is mediated by the Higgs boson and decays promptly to a lepton pair [18,19]. This analysis expands the search to a more general case to include a possible new scalar $(\Phi)$ that couples to $Z$ and $Z_{d}$, instead of only the Higgs boson, and considers the scenario in which the $Z_{d}$ decays hadronically with a $c \tau$ between a few centimeters and 100 meters, where $c$ is the speed of light and $\tau$ is the $Z_{d}$ proper lifetime.

The analysis uses data from $\sqrt{s}=13 \mathrm{TeV}$ protonproton $(p p)$ collisions at the LHC that were recorded by the ATLAS detector in 2015 and 2016 with single-electron and single-muon triggers [20], corresponding to an integrated luminosity of $36.1 \pm 0.8 \mathrm{fb}^{-1}$. The ATLAS detector [21] is a multipurpose particle detector with a cylindrical geometry [22]. The distance between two objects in the $\eta-\phi$ space is $\Delta R=\sqrt{(\Delta \eta)^{2}+(\Delta \phi)^{2}}$. Transverse momentum is defined by $p_{T}=p \sin \theta$. It consists of an inner detector (ID) [23] surrounded by a solenoid that produces a $2 \mathrm{~T}$ magnetic field, electromagnetic and hadronic calorimeters, and a muon spectrometer in a magnetic field produced by a system of toroid magnets. The ID measures the trajectories of charged particles over the full azimuthal angle and in a pseudorapidity range of $|\eta|<2.5$ using silicon pixel, silicon microstrip, and straw-tube transitionradiation tracker detectors. Liquid-argon electromagnetic calorimeters (LArCal) extend from 1.5 to $2.0 \mathrm{~m}$ in radius in the barrel and from 3.6 to $4.25 \mathrm{~m}$ in $|z|$ in the end caps. A scintillator-tile calorimeter (TileCal) provides hadronic calorimetery and covers the region $2.25<r<4.25 \mathrm{~m}$. 
The experimental signature searched for is the $Z_{d}$ decaying within the TileCal, thus producing a jet that has little or no energy deposited in the LArCal, and no charged tracks that point to the reconstructed location of the collision of interest (hereafter called the primary vertex).

Monte Carlo (MC) simulated events are used to optimize the event selection and to help validate the analysis. Signal samples were generated using the PYTHIA 8.210 [24] generator with the NNPDF23LO parton distribution functions (PDFs) [25] and the A14 set of tuned parameters (A14 tune) [26], with an assumption that the $Z_{d}$ decays only to the highest-mass heavy quark pair $(b \bar{b}$ or $c \bar{c})$ that is kinematically allowed. Nine samples were produced with three different $Z_{d}$ masses for each of three $\Phi$ masses $\left(m_{Z_{d}}=\{5,10,15\},\{10,50,100\}\right.$, and $\{20,100,200\}$ for $m_{\Phi}=125,250$, and $500 \mathrm{GeV}$, respectively), where $m_{\Phi}=$ $125 \mathrm{GeV}$ corresponds to the SM Higgs boson. The $c \tau$ of the $Z_{d}$ is a free parameter in this model. For each mass hypothesis of $Z_{d}$ and $\Phi$, its $c \tau$ is chosen to maximize the probability for $Z_{d}$ to decay inside the TileCal, which is found to be around $20 \%$ for all samples, as shown in Fig. 1(a). The events were reweighted to produce samples with different $c \tau\left(Z_{d}\right)$ [8] between 0.01 and $100 \mathrm{~m}$. The dominant SM background arises from events with a $Z$ boson produced in association with jets $(Z+$ jets), where a jet mimics the experimental signature of $Z_{d}$ decay inside the TileCal due to the presence of long-lived SM particles $\left(K_{L}^{0}\right.$, $\Lambda$, etc), out-of-time pileup (additional $p p$ collisions occurring in bunch-crossings just before and after the collision of interest), noise, detector inefficiencies, and beam-induced background. Additional SM background processes include the production of top quarks and $W+$ jets. The SM background $\mathrm{MC}$ samples are generated with the configurations described in Ref. [27] for $W+$ jets and $Z+$ jets production, and Ref. [28] for $t \bar{t}$ and single top production. The effect of multiple $p p$ interactions in the same and neighboring bunch crossings (pileup) is included by overlaying minimum-bias events simulated with PYTHIA8.186 on each generated event in all samples. The generated samples were processed through a GEANT4-based detector simulation $[29,30]$ and the standard ATLAS reconstruction software.

The selected events have a pair of oppositely charged and isolated electrons [31] or muons [32] to form a $Z$ boson candidate. Electrons and muons are required to have $|\eta|<$ 2.47 and $|\eta|<2.4$, respectively, and $p_{\mathrm{T}}>25 \mathrm{GeV}$ $(27 \mathrm{GeV})$ in data collected in 2015 (2016). The invariant mass of the $Z$ candidate $\left(m_{\ell \ell}\right)$ is required to be between 66 and $116 \mathrm{GeV}$. Selected jets must have transverse energy $E_{\mathrm{T}}>40 \mathrm{GeV}$ and $|\eta|<2.0$ to ensure the jets are completely within the ID. They are reconstructed using the anti$k_{t}$ algorithm $[33,34]$ with a radius parameter $R=0.4$ and calibrated to particle level [35]. Standard ATLAS jetquality criteria [36] are applied, except the one for the ratio of the energy deposited in the hadronic calorimeter to the total energy since it removes signal jets. A jet is considered as a $Z_{d}$ candidate, referred to as a calorimeterratio jet (CR jet) hereafter, if it satisfies $\log _{10}\left(E_{\text {Tile }} / E_{\mathrm{LAr}}\right)>$ 1.2 with no associated tracks [37] of $p_{\mathrm{T}}>1 \mathrm{GeV}$ originating from the primary vertex, where $E_{\text {Tile }}$ and $E_{\mathrm{LAr}}$ are the jet energy deposited in the TileCal and LArCal, respectively [6], as shown in Fig. 1(b). Jets with $E_{\mathrm{T}}<60 \mathrm{GeV}$ in the transition region between the barrel and end cap cryostats $(1.0<|\eta|<1.3)$ are not considered as CR-jet candidates due to noise in the gap scintillator of the TileCal [38]. In addition, the timing of the CR jet is required to be between -3 and $15 \mathrm{~ns}$ in order to suppress jets arising from out-of-time pileup and beam-induced backgrounds [6]. The timing of a jet is obtained from its constituent calorimeter cells by calculating an average time over cells weighted by cell energy squared where the cell time is measured
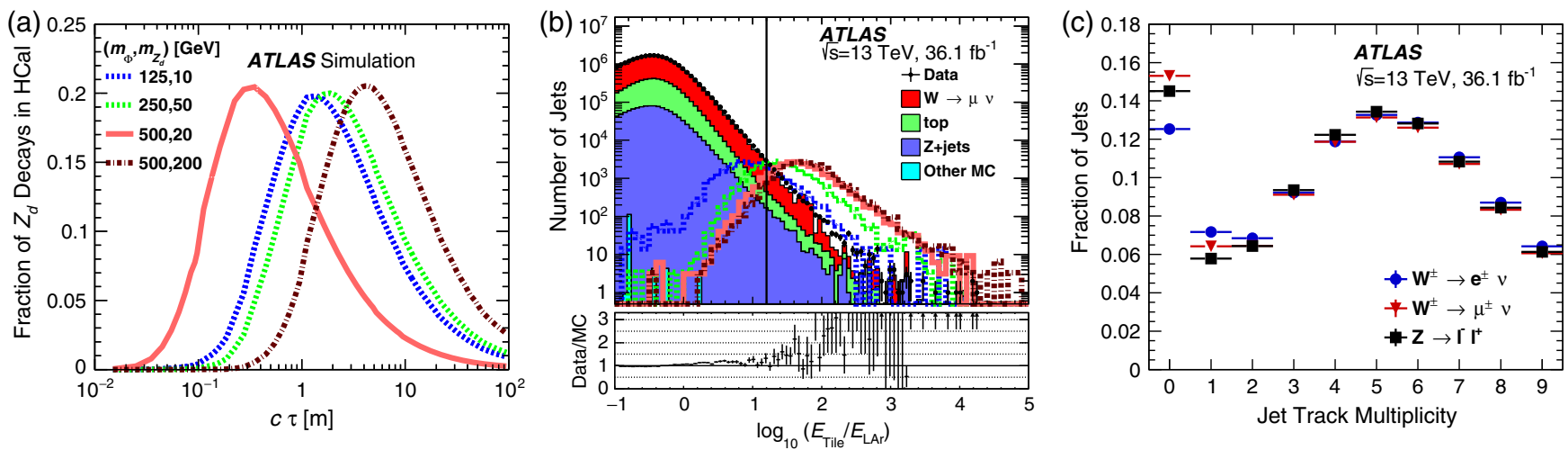

FIG. 1. (a) The probability of a $Z_{d}$ boson to decay within the TileCal as a function of the $c \tau$ for each choice of $m_{\Phi}$ and $m_{Z_{d}}$. As $m_{Z_{d}}$ increases (for a fixed $m_{\Phi}$ ) the $Z_{d}$ becomes less boosted and therefore travels less distance into the detector before decaying. (b) The distributions of $\log _{10}\left(E_{\text {Tile }} / E_{\mathrm{LAr}}\right)$ for jets in background and signal MC simulations [see legend of Fig. 1(a) for signal labels] and $W+$ jets data (prior to any requirements on the track multiplicity of jets or jet timing). The threshold for this variable is shown as a solid black line. (c) The distributions of the track multiplicity for jets prior to the selection of CR jets in the $W+$ jets and $Z+$ jets data samples. 
according to the bunch crossing clock, relative to the expected time of flight from the bunch crossing to the cell [39]. After this selection, the number of selected events containing a $\mathrm{CR}$ jet with an $E_{\mathrm{T}}$ above a chosen threshold is compared with the predicted total number of background events. The minimum $E_{\mathrm{T}}$ requirement of the selected CR jets is further optimized to achieve the highest experimental sensitivity for each mass hypothesis [40]. It is set to be $40 \mathrm{GeV}$ for $m_{\Phi}=125 \mathrm{GeV}$ samples, $60 \mathrm{GeV}$ for $m_{\Phi}=$ $250 \mathrm{GeV}$ samples, and $80 \mathrm{GeV}$ for $m_{\Phi}=500 \mathrm{GeV}$ samples.

The signal efficiency times acceptance $(\epsilon \times A)$ is defined as the ratio of the number of selected signal events in $\mathrm{MC}$ simulations to the number of generated signal events. It is a function of $m_{\Phi}, m_{Z_{d}}$, and the $c \tau\left(Z_{d}\right)$. The maximum values vary between approximately $1 \%$ for lowest $m_{\Phi}$ samples to $5-7 \%$ for samples with larger $\Phi$ mass. The main loss is due to the low probability that $Z_{d}$ decays inside the TileCal, as shown in Fig. 1(a). The samples for $m_{\Phi}=125 \mathrm{GeV}$ suffer further efficiency loss due to the jet $E_{\mathrm{T}}$ requirement.

MC simulations are not reliable enough to estimate the backgrounds of this analysis, as illustrated by the righthand side of Fig. 1(b). A data-driven approach is thus used for its estimation. A control data sample of SM $W+$ jets events, with the same event selection criteria of $W \rightarrow$ $\ell \nu(\ell=e, \mu)$ in Ref. [41], is used to derive the probability for a jet to pass the selection of the CR jet, assuming that the $Z_{d}$ cannot be produced in association with a $W$ boson. The probability is calculated as $f_{\mathrm{CR}}=N_{\mathrm{CRjet}} / N_{\text {jet }}$ in bins of the jet $E_{\mathrm{T}}$ and $\eta$, where $N_{\mathrm{CRjet}}$ is the number of jets that satisfy the CR-jet selection criteria and $N_{\text {jet }}$ is the total number of jets from the $W+$ jets sample in each bin, as summarized in Table I. For a selected event in data containing a $Z \rightarrow \ell \ell$ candidate and $N$ jets, the corresponding probability for it to be identified as a signal event is therefore $P=1-\prod_{i \in N}\left[1-f_{\mathrm{CR}}\left(E_{\mathrm{T}}{ }^{i}, \eta^{i}\right)\right]$, where $f_{\mathrm{CR}}\left(E_{\mathrm{T}}{ }^{i}, \eta^{i}\right)$ is the probability of the $i$ th jet in the event to satisfy the CRjet selection criteria. The sum of the probabilities $P$ for all the selected events is therefore the expected number of background events. Potential signal contamination of this control region was estimated using $\mathrm{MC}$ and found to have a $<1 \%$ impact on the background estimate.

TABLE I. The numbers of jets satisfying different requirements on minimum jet $E_{\mathrm{T}}$ and their corresponding averaged CR-jet selection probabilities in the $W \rightarrow \ell \nu$ samples.

\begin{tabular}{lccc}
\hline \hline Minimum jet $E_{\mathrm{T}}$ & $40 \mathrm{GeV}$ & $60 \mathrm{GeV}$ & $80 \mathrm{GeV}$ \\
\hline$N_{\mathrm{CRjet}}(W \rightarrow e \nu)$ & 982 & 189 & 63 \\
$N_{\mathrm{CRjet}}(W \rightarrow \mu \nu)$ & 1030 & 186 & 71 \\
$N_{\text {jet }}(W \rightarrow e \nu)$ & $3.3 \times 10^{7}$ & $1.5 \times 10^{7}$ & $0.8 \times 10^{7}$ \\
$N_{\text {jet }}(W \rightarrow \mu \nu)$ & $3.1 \times 10^{7}$ & $1.3 \times 10^{7}$ & $0.7 \times 10^{7}$ \\
$f_{\mathrm{CR}}(W \rightarrow e \nu)$ & $3.0 \times 10^{-5}$ & $1.3 \times 10^{-5}$ & $7.9 \times 10^{-6}$ \\
$f_{\mathrm{CR}}(W \rightarrow \mu \nu)$ & $3.3 \times 10^{-5}$ & $1.4 \times 10^{-5}$ & $9.7 \times 10^{-6}$ \\
\hline \hline
\end{tabular}

Studies [6] have shown that jets originating from quarks and gluons may have different probabilities of satisfying the selection criteria for $\mathrm{CR}$ jets. MC simulations predict that jets from $W+$ jets and $Z+$ jets production are mostly initiated by quarks with a similar fraction $(\sim 73 \%)$. However, $W+$ jets data samples are contaminated with a significant fraction of SM multijet events with a misidentified lepton, which is estimated to be approximately $2 \%$ in the muon final state and $17 \%$ in the electron final state using background-enriched control samples [41]. SM multijets originate primarily from gluons and thus introduce a difference between the $W+$ jets and $Z+$ jets samples. The distributions of the track multiplicity of a jet in the $W+$ jets and $Z+$ jets samples, which are sensitive to the quark/gluon jet fraction [42], show a significant difference for track multiplicities of 0 and 1 in Fig. 1(c). As a result, the $f_{\mathrm{CR}}$ values measured in the muon final state are used for the central value of the background estimate, while the $f_{\mathrm{CR}}$ values measured in the electron final state are used as a cross-check to assign a systematic uncertainty due to different quark or gluon jet fractions in the $W+$ jets and $Z+$ jets samples. The measured probabilities, $f_{\mathrm{CR}}$, are found to be dependent on the jet multiplicity in the event. Studies show that this is caused by the presence of jets from pileup interactions which deposit additional energy in the LArCal, suppressing the signature of CR jets. The jet multiplicity and pileup distributions of events in the $W+$ jets sample are the same as those from the $Z+$ jets sample, and therefore the parametrization of the measured $f_{\mathrm{CR}}$ as a function of jet multiplicity or pileup is not necessary.

Several studies were performed to validate the background estimation procedure. $\mathrm{A} Z+$ jets sideband is formed from events satisfying all signal selection criteria except the invariant-mass requirement for the $Z$ candidate. The mass is required to be $30<m_{\ell \ell}<55 \mathrm{GeV}$. The events in the higher mass sideband $m_{\ell \ell}>116 \mathrm{GeV}$ are not used as they are still dominated by $Z+$ jets production, as indicated by background MC simulations [43]. Based on the measured CR-jet probability in $W+$ jets, the expected numbers of background events with $E_{\mathrm{T}}$ of CR-jets greater than 40,60, and $80 \mathrm{GeV}$ are estimated to be $2.2 \pm 0.2,0.7 \pm 0.1$, and $0.3 \pm 0.1$, where the uncertainties are statistical only. They are consistent with the corresponding observations in data, which have 1,1 , and 0 events, respectively.

The background estimation method relies on an assumption that jets in the $W+$ jets sample have the same characteristics as jets in the $Z+$ jets sample. This assumption is tested using validation jets that are defined to satisfy the selection criteria of the CR jets except the zero-ghost-track requirement. Validation jets must have more than two associated tracks to avoid signal contamination, as MC-simulated signal events show that less than $1 \%$ of jets from $Z_{d}$ decays inside the TileCal have more than two tracks. The probability for a jet to be identified as 
TABLE II. Event yields for the predicted backgrounds and data, and the expected and observed ULs on the signal yields at the $95 \%$ C.L. The quoted errors include both the statistical and systematic uncertainties.

\begin{tabular}{lccc}
\hline \hline Minimum jet $E_{\mathrm{T}}$ & $40 \mathrm{GeV}$ & $60 \mathrm{GeV}$ & $80 \mathrm{GeV}$ \\
\hline Background & $175 \pm 22$ & $33.0 \pm 4.4$ & $13.2 \pm 3.5$ \\
Data & 158 & 35 & 16 \\
Expected UL & 65 & 17 & 10 \\
Observed UL & 50 & 18 & 13 \\
\hline \hline
\end{tabular}

a validation jet is measured in the $W+$ jets sample as a function of jet $E_{\mathrm{T}}$ and $\eta$ and subsequently used to predict the number of events containing a $Z \rightarrow \ell \ell$ candidate and at least one validation jet. As a result, a global scale factor of 1.24 , which is defined as the observed number of events with validation jets divided by the predicted value, is applied to the measured probabilities $f_{\mathrm{CR}}$. A $50 \%$ relative correction of the scale factor $( \pm 0.12)$ is assigned as a systematic uncertainty due to potential bias of the background estimation procedure.

The systematic uncertainties of the background estimation include the statistical uncertainty from the $W+$ jets sample (2-8\%), potential difference in the quark or gluon jet fractions between the $W+$ jets and $Z+$ jets samples (7$20 \%)$, and the scale factor uncertainty $(\sim 10 \%)$ measured using the validation jets. The uncertainty of the integrated luminosity is $2.1 \%[44,45]$. Uncertainties resulting from detector effects such as the trigger efficiencies, the energy scale and resolution of jets [35], lepton identification, reconstruction and isolation efficiencies, lepton momentum scales, and resolutions $[31,32,46]$ only affect the calculation of the selection efficiencies of $Z_{d}$ signal events, since the background is estimated from the data. They are typically small $(<1-5 \%)$. Pileup adds extra tracks and electromagnetic energy to jets. The systematic uncertainties associated with reweighting the pileup distribution from the generated MC simulations to the data are typically small $(<5 \%)$ except for the samples with $m_{\Phi}=125 \mathrm{GeV}$ $(\sim 13 \%)$, in which case the $Z_{d}$ have small energies and additional energy deposition in the LArCal from pileup can significantly affect their selection efficiencies. Since the $\mathrm{CR}$ jets in this analysis have a very small fraction of their energies inside the LArCal, the in situ jet energy intercalibration $[6,35]$ is repeated using the $p_{\mathrm{T}}$ balance method in dijets events, and the observed difference between the data and MC simulation is used to derive an additional systematic uncertainty of the jet energy scale. The corresponding effect on the signal efficiencies is approximately 5-9\% for samples with $m_{\Phi}=125 \mathrm{GeV}$, and negligible for samples with higher $m_{\Phi}$ values. The effects on the signal efficiency and acceptance due to theoretical uncertainties, such as a PDF choice and initial- and final-state radiation modeling, are found to be very small $(<1 \%)$.

Table II shows the predicted numbers of background events and the observed data events with different minimum $E_{\mathrm{T}}$ requirements for the selected CR jets. The data are well described by the background estimate. In the absence of any significant data excess, upper limits (ULs) on the signal yield of $p p \rightarrow \Phi \rightarrow Z Z_{d}$ at the $95 \%$ confidence level (C.L.) are derived using the C.L.s method [40] taking into account both the statistical and systematic uncertainties. The results are listed in Table II.

The results are further reinterpreted as the UL on the production cross section of $\Phi$ times the decay branching fraction $B\left(\Phi \rightarrow Z Z_{d}\right)$, as a function of $m_{\Phi}, m_{Z_{d}}$, and $c \tau$ of the $Z_{d}$. In the case of the SM Higgs boson, where $m_{H}=125 \mathrm{GeV}$, the $\mathrm{UL}$ on $B\left(H \rightarrow Z Z_{d}\right)$ are evaluated using the SM Higgs boson cross section $\sigma_{\mathrm{SM}}=48.5_{-6.7}^{+4.6} \mathrm{pb}$ [47] of the gluon-gluon fusion process; other production modes are ignored. The results, reweighted to other $c \tau$ [8], are shown in Fig. 2.

In conclusion, this Letter reports a novel search for a singly produced long-lived neutral particle $Z_{d}$, in association with an SM $Z$ boson via coupling to an intermediate
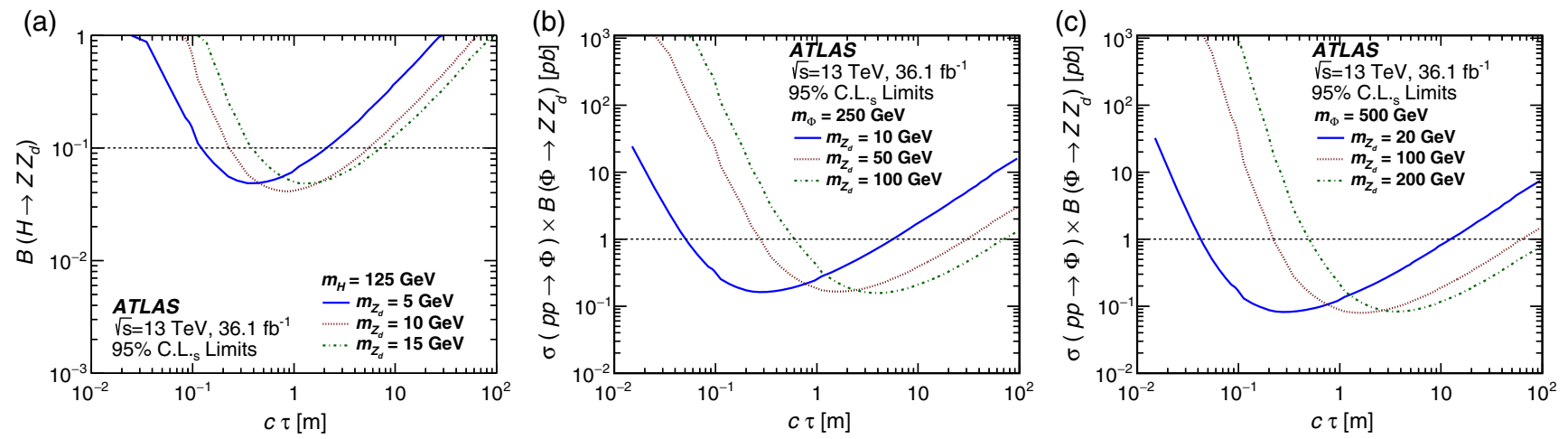

FIG. 2. (a) Observed $95 \%$ C.L. limits on the decay branching fraction of $B\left(H \rightarrow Z Z_{d}\right)$ for the SM Higgs boson as a function of the $c \tau\left(Z_{d}\right)$. (b) and (c) Observed $95 \%$ C.L. limits on the production cross section $(\sigma)$ of $\Phi$ times its decay branching fraction to $Z Z_{d}$ as a function of the $c \tau\left(Z_{d}\right)$. 
scalar boson. The analysis is based on $36.1 \pm 0.8 \mathrm{fb}^{-1}$ of $p p$ collisions at $\sqrt{s}=13 \mathrm{TeV}$ collected in 2015 and 2016 with the ATLAS detector at the LHC. No excess over the expected background was observed. Upper limits on the production cross section of the scalar boson times its branching fraction to the long-lived neutral particle at $95 \%$ C.L. are derived as a function of the particle proper lifetimes for different masses of the scalar boson and the $Z_{d}$. In the case that the intermediate scalar boson is the SM Higgs boson, its decay branching fraction to a long-lived neutral particle with a $c \tau$ approximately between 0.1 and $7 \mathrm{~m}$ is excluded with a 95\% C.L. up to $10 \%$ for $m_{Z_{d}}$ between 5 and $15 \mathrm{GeV}$.

We thank CERN for the very successful operation of the LHC, as well as the support staff from our institutions without whom ATLAS could not be operated efficiently. We acknowledge the support of ANPCyT, Argentina; YerPhI, Armenia; ARC, Australia; BMWFW and FWF, Austria; ANAS, Azerbaijan; SSTC, Belarus; CNPq and FAPESP, Brazil; NSERC, NRC and CFI, Canada; CERN; CONICYT, Chile; CAS, MOST, and NSFC, China; COLCIENCIAS, Colombia; MSMT CR, MPO CR, and VSC CR, Czech Republic; DNRF and DNSRC, Denmark; IN2P3-CNRS, CEA-DRF/IRFU, France; SRNSFG, Georgia; BMBF, HGF, and MPG, Germany; GSRT, Greece; RGC, Hong Kong SAR, China; ISF and Benoziyo Center, Israel; INFN, Italy; MEXT and JSPS, Japan; CNRST, Morocco; NWO, Netherlands; RCN, Norway; MNiSW and NCN, Poland; FCT, Portugal; MNE/IFA, Romania; MES of Russia and NRC KI, Russian Federation; JINR; MESTD, Serbia; MSSR, Slovakia; ARRS and MIZŠ, Slovenia; DST/NRF, South Africa; MINECO, Spain; SRC and Wallenberg Foundation, Sweden; SERI, SNSF and Cantons of Bern and Geneva, Switzerland; MOST, Taiwan; TAEK, Turkey; STFC, United Kingdom; DOE and NSF, United States of America. In addition, individual groups and members have received support from BCKDF, CANARIE, CRC, and Compute Canada, Canada; COST, ERC, ERDF, Horizon 2020, and Marie Skłodowska-Curie Actions, European Union; Investissements d' Avenir Labex and Idex, ANR, France; DFG and AvH Foundation, Germany; Herakleitos, Thales, and Aristeia programmes co-financed by EU-ESF and the Greek NSRF, Greece; BSF-NSF and GIF, Israel; CERCA Programme Generalitat de Catalunya, Spain; The Royal Society and Leverhulme Trust, United Kingdom. The crucial computing support from all WLCG partners is acknowledged gratefully, in particular from CERN, the ATLAS Tier-1 facilities at TRIUMF (Canada), NDGF (Denmark, Norway, Sweden), CC-IN2P3 (France), KIT/ GridKA (Germany), INFN-CNAF (Italy), NL-T1 (Netherlands), PIC (Spain), ASGC (Taiwan), RAL (UK), and BNL (USA), the Tier-2 facilities worldwide and large non-WLCG resource providers. Major contributors of computing resources are listed in Ref. [48].
[1] S. Dimopoulos, M. Dine, S. Raby, and S. D. Thomas, Experimental Signatures of Low Energy Gauge-Mediated Supersymmetry Breaking, Phys. Rev. Lett. 76, 3494 (1996).

[2] R. Barbier et al., R-parity violating supersymmetry, Phys. Rep. 420, 1 (2005).

[3] D. Smith and N. Weiner, Inelastic dark matter, Phys. Rev. D 64, 043502 (2001).

[4] M. J. Strassler and K. M. Zurek, Echoes of a hidden valley at hadron colliders, Phys. Lett. B 651, 374 (2007).

[5] M. J. Strassler and K. M. Zurek, Discovering the Higgs through highly-displaced vertices, Phys. Lett. B 661, 263 (2008).

[6] ATLAS Collaboration, Search for pair-produced long-lived neutral particles decaying to jets in the ATLAS hadronic calorimeter in $p p$ collisions at $\sqrt{s}=8 \mathrm{TeV}$, Phys. Lett. B 743, 15 (2015).

[7] ATLAS Collaboration, Search for long-lived, weakly interacting particles that decay to displaced hadronic jets in proton-proton collisions at $\sqrt{s}=8 \mathrm{TeV}$ with the ATLAS detector, Phys. Rev. D 92, 012010 (2015).

[8] ATLAS Collaboration, Search for massive, long-lived particles using multitrack displaced vertices or displaced lepton pairs in $p p$ collisions at $\sqrt{s}=8 \mathrm{TeV}$ with the ATLAS detector, Phys. Rev. D 92, 072004 (2015).

[9] ATLAS Collaboration, Search for long-lived, massive particles in events with displaced vertices and missing transverse momentum in $\sqrt{s}=13 \mathrm{TeV} p p$ collisions with the ATLAS detector, Phys. Rev. D 97, 052012 (2018).

[10] CMS Collaboration, Search for long-lived neutral particles decaying to quark-antiquark pairs in proton-proton collisions at $\sqrt{s}=8 \mathrm{TeV}$, Phys. Rev. D 91, 012007 (2015).

[11] CMS Collaboration, Search for new long-lived particles at $\sqrt{s}=13 \mathrm{TeV}$, Phys. Lett. B 780, 432 (2018).

[12] LHCb Collaboration, Search for long-lived particles decaying to jet pairs, Eur. Phys. J. C 75, 152 (2015).

[13] LHCb Collaboration, Search for massive long-lived particles decaying semileptonically in the LHCb detector, Eur. Phys. J. C 77, 224 (2017).

[14] CDF Collaboration, Search for heavy metastable particles decaying to jet pairs in $p \bar{p}$ collisions at $\sqrt{s}=1.96 \mathrm{TeV}$, Phys. Rev. D 85, 012007 (2012).

[15] D0 Collaboration, Search for Resonant Pair Production of Neutral Long-Lived Particles Decaying to $b \bar{b}$ in $p \bar{p}$ Collisions at $\sqrt{s}=1.96 \mathrm{TeV}$, Phys. Rev. Lett. 103, 071801 (2009).

[16] H. Davoudiasl, H.-S. Lee, and W. J. Marciano, "Dark" Z implications for parity violation, rare meson decays, and Higgs physics, Phys. Rev. D 85, 115019 (2012).

[17] H. Davoudiasl, H.-S. Lee, I. Lewis, and W. J. Marciano, Higgs decays as a window into the dark sector, Phys. Rev. D 88, 015022 (2013).

[18] ATLAS Collaboration, Search for new light gauge bosons in Higgs boson decays to four-lepton final states in $p p$ collisions at $\sqrt{s}=8 \mathrm{TeV}$ with the ATLAS detector at the LHC, Phys. Rev. D 92, 092001 (2015).

[19] ATLAS Collaboration, Search for Higgs boson decays to beyond-the-Standard-Model light bosons in four-lepton events with the ATLAS detector at $\sqrt{s}=13 \mathrm{TeV}$, J. High Energy Phys. 06 (2018) 166. 
[20] ATLAS Collaboration, Performance of the ATLAS trigger system in 2015, Eur. Phys. J. C 77, 317 (2017).

[21] ATLAS Collaboration, The ATLAS experiment at the CERN large hadron collider, J. Instrum. 3, S08003 (2008).

[22] ATLAS uses a right-handed coordinate system with its origin at the nominal interaction point (IP) in the center of the detector and the $z$ axis along the beam pipe. The $x$ axis points to the center of the LHC ring, and the $y$ axis points upward. Cylindrical coordinates $(r, \phi)$ are used in the transverse plane, $\phi$ being the azimuthal angle around the $z$ axis. The pseudorapidity is defined in terms of the polar angle $\theta$ as $\eta=-\ln \tan (\theta / 2)$.

[23] B. Abbott et al., Production and Integration of the ATLAS insertable B-layer, J. Instrum. 13, T05008 (2018).

[24] T. Sjöstrand, S. Ask, J. R. Christiansen, R. Corke, N. Desai, P. Ilten, S. Mrenna, S. Prestel, C. O. Rasmussen, and P. Z. Skands, An introduction to PYTHIA 8.2, Comput. Phys. Commun. 191, 159 (2015).

[25] R. D. Ball et al., Parton distributions for the LHC Run II, J. High Energy Phys. 04 (2015) 040.

[26] ATLAS Collaboration, ATLAS Pythia 8 tunes to $7 \mathrm{TeV}$ data CERN Report No. ATL-PHYS-PUB-2014-021, 2014, https://cds.cern.ch/record/1966419.

[27] ATLAS Collaboration, Monte Carlo generators for the production of a $W$ or $Z / \gamma^{*}$ Boson in association with jets at ATLAS in Run 2, CERN Report No. ATL-PHYS-PUB2016-003, 2016, https://cds.cern.ch/record/2120133.

[28] ATLAS Collaboration, Simulation of top-quark production for the ATLAS experiment at $\sqrt{s}=13 \mathrm{TeV}$, CERN Report No. ATL-PHYS-PUB-2016-004, 2016, https://cds.cern.ch/ record/2120417.

[29] S. Agostinelli et al., GEANT4-A simulation toolkit, Nucl. Instrum. Methods Phys. Res., Sect. A 506, 250 (2003).

[30] ATLAS Collaboration, The ATLAS simulation infrastructure, Eur. Phys. J. C 70, 823 (2010).

[31] ATLAS Collaboration, Electron efficiency measurements with the ATLAS detector using the 2015 LHC protonproton collision data, CERN Report No. ATLAS-CONF2016-024, 2016, https://cds.cern.ch/record/2157687.

[32] ATLAS Collaboration, Muon reconstruction performance of the ATLAS detector in proton-proton collision data at $\sqrt{s}=13$ TeV, Eur. Phys. J. C 76, 292 (2016).

[33] M. Cacciari, G. P. Salam, and G. Soyez, The anti-k $t_{t}$ jet clustering algorithm, J. High Energy Phys. 04 (2008) 063.

[34] M. Cacciari, G. P. Salam, and G. Soyez, FastJet user manual, Eur. Phys. J. C 72, 1896 (2012).
[35] ATLAS Collaboration, Jet energy scale measurements and their systematic uncertainties in proton-proton collisions at $\sqrt{s}=13 \mathrm{TeV}$ with the ATLAS detector, Phys. Rev. D 96, 072002 (2017).

[36] ATLAS Collaboration, Selection of jets produced in $13 \mathrm{TeV}$ proton-proton collisions with the ATLAS detector, CERN, Report No. ATLAS-CONF-2015-029, 2015, https://cds .cern.ch/record/2037702.

[37] M. Cacciari and G. P. Salam, Pileup subtraction using jet areas, Phys. Lett. B 659, 119 (2008).

[38] ATLAS Collaboration, Search for long-lived neutral particles decaying into displaced lepton-jets in proton-proton collisions at $\sqrt{s}=13 \mathrm{TeV}$ with the ATLAS detector, CERN Report No. ATLAS-CONF-2016-042, 2016, https://cds.cern.ch/record/2206083.

[39] ATLAS Collaboration, Topological cell clustering in the ATLAS calorimeters and its performance in LHC Run 1, Eur. Phys. J. C 77, 490 (2017).

[40] A. L. Read, Presentation of search results: The $\mathrm{CL}_{s}$ technique, J. Phys. G 28, 2693 (2002).

[41] ATLAS Collaboration, Measurement of $W^{ \pm}$and $Z$-boson production cross sections in $p p$ collisions at $\sqrt{s}=13 \mathrm{TeV}$ with the ATLAS detector, Phys. Lett. B 759, 601 (2016).

[42] ATLAS Collaboration, Quark versus gluon jet tagging using charged-particle constituent multiplicity with the ATLAS detector, CERN Report No. ATL-PHYS-PUB-2017-009, 2017, https://cds.cern.ch/record/2263679.

[43] ATLAS Collaboration, Measurement of the Drell-Yan triple-differential cross section in $p p$ collisions at $\sqrt{s}=8 \mathrm{TeV}$, J. High Energy Phys. 12 (2017) 059.

[44] ATLAS Collaboration, Luminosity determination in $p p$ collisions at $\sqrt{s}=8 \mathrm{TeV}$ using the ATLAS detector at the LHC, Eur. Phys. J. C 76, 653 (2016).

[45] G. Avoni et al., The new LUCID-2 detector for luminosity measurement and monitoring in ATLAS, J. Instrum. 13, P07017 (2018).

[46] ATLAS Collaboration, Electron and photon energy calibration with the ATLAS detector using data collected in 2015 at $\sqrt{s}=13 \mathrm{TeV}$, CERN Report No. ATL-PHYS-PUB-2016015, 2016, https://cds.cern.ch/record/2203514.

[47] D. de Florian et al., Handbook of LHC Higgs cross sections: 4. Deciphering the Nature of the Higgs sector, arXiv: 1610.07922 .

[48] ATLAS Collaboration, ATLAS computing acknowledgements, CERN Report No. ATL-GEN-PUB-2016-002, 2016, https://cds.cern.ch/record/2202407.

M. Aaboud, ${ }^{34 \mathrm{~d}}$ G. Aad, ${ }^{99}$ B. Abbott, ${ }^{125}$ O. Abdinov, ${ }^{13, a}$ B. Abeloos, ${ }^{129}$ D. K. Abhayasinghe, ${ }^{91}$ S. H. Abidi, ${ }^{164}$ O. S. AbouZeid, ${ }^{39}$ N. L. Abraham, ${ }^{153}$ H. Abramowicz, ${ }^{158}$ H. Abreu, ${ }^{157}$ Y. Abulaiti, ${ }^{6}$ B. S. Acharya, ${ }^{64 a, 64 b, b}$ S. Adachi, ${ }^{160}$ L. Adam,${ }^{97}$ L. Adamczyk, ${ }^{81 a}$ J. Adelman, ${ }^{119}$ M. Adersberger, ${ }^{12}$ A. Adiguzel, ${ }^{12 c, c}$ T. Adye, ${ }^{141}$ A. A. Affolder, ${ }^{143}$ Y. Afik, ${ }^{157}$ C. Agheorghiesei ${ }^{27 \mathrm{c}}$ J. A. Aguilar-Saavedra, ${ }^{137 f, 137 \mathrm{a}, \mathrm{d}}$ F. Ahmadov, ${ }^{77, \mathrm{e}}$ G. Aielli, ${ }^{71 \mathrm{a}, 71 \mathrm{~b}}$ S. Akatsuka, ${ }^{83}$ T. P. A. Åkesson, ${ }^{94}$ E. Akilli, ${ }^{52}$ A. V. Akimov, ${ }^{108}$ G. L. Alberghi, ${ }^{23 b, 23 a}$ J. Albert, ${ }^{173}$ P. Albicocco, ${ }^{49}$ M. J. Alconada Verzini, ${ }^{86}$

S. Alderweireldt, ${ }^{117}$ M. Aleksa, ${ }^{35}$ I. N. Aleksandrov, ${ }^{77}$ C. Alexa, ${ }^{27 b}$ D. Alexandre, ${ }^{19}$ T. Alexopoulos, ${ }^{10}$ M. Alhroob, ${ }^{125}$ B. Ali, ${ }^{139}$ G. Alimonti, ${ }^{66 a}$ J. Alison, ${ }^{36}$ S. P. Alkire, ${ }^{145}$ C. Allaire, ${ }^{129}$ B. M. M. Allbrooke, ${ }^{153}$ B. W. Allen, ${ }^{128}$ P. P. Allport,${ }^{21}$ A. Aloisio, ${ }^{67 a, 67 b}$ A. Alonso, ${ }^{39}$ F. Alonso,${ }^{86}$ C. Alpigiani, ${ }^{145}$ A. A. Alshehri, ${ }^{55}$ M. I. Alstaty, ${ }^{99}$ B. Alvarez Gonzalez, ${ }^{35}$ 
D. Álvarez Piqueras, ${ }^{171}$ M. G. Alviggi, ${ }^{67,67 b}$ B. T. Amadio, ${ }^{18}$ Y. Amaral Coutinho, ${ }^{78 b}$ A. Ambler, ${ }^{101}$ L. Ambroz, ${ }^{132}$ C. Amelung, ${ }^{26}$ D. Amidei, ${ }^{103}$ S. P. Amor Dos Santos, ${ }^{137 a, 137 \mathrm{c}}$ S. Amoroso,${ }^{44}$ C. S. Amrouche,${ }^{52}$ F. An,${ }^{76}$ C. Anastopoulos, ${ }^{146}$ L. S. Ancu,${ }^{52}$ N. Andari, ${ }^{142}$ T. Andeen, ${ }^{11}$ C. F. Anders,${ }^{59 b}$ J. K. Anders, ${ }^{20}$ K. J. Anderson, ${ }^{36}$ A. Andreazza, ${ }^{66 a, 66 b}$ V. Andrei, ${ }^{59 a}$ C. R. Anelli, ${ }^{173}$ S. Angelidakis,${ }^{37}$ I. Angelozzi, ${ }^{118}$ A. Angerami, ${ }^{38}$ A. V. Anisenkov, ${ }^{120 b, 120 a}$ A. Annovi,${ }^{69 a}$ C. Antel, ${ }^{59 a}$ M. T. Anthony, ${ }^{146}$ M. Antonelli, ${ }^{49}$ D. J. A. Antrim, ${ }^{168}$ F. Anulli, ${ }^{70 a}$ M. Aoki, ${ }^{79}$ J. A. Aparisi Pozo, ${ }^{171}$ L. Aperio Bella, ${ }^{35}$ G. Arabidze, ${ }^{104}$ J. P. Araque, ${ }^{137}$ V. Araujo Ferraz ${ }^{78 b}$ R. Araujo Pereira, ${ }^{78 b}$ A. T. H. Arce, ${ }^{47}$ R. E. Ardell, ${ }^{91}$ F. A. Arduh ${ }^{86}$ J-F. Arguin, ${ }^{107}$ S. Argyropoulos, ${ }^{75}$ J.-H. Arling, ${ }^{44}$ A. J. Armbruster, ${ }^{35}$ L. J. Armitage, ${ }^{90}$ A. Armstrong, ${ }^{168}$ O. Arnaez, ${ }^{164}$ H. Arnold, ${ }^{118}$ M. Arratia, ${ }^{31}$ O. Arslan, ${ }^{24}$ A. Artamonov, ${ }^{109, a}$ G. Artoni, ${ }^{132}$ S. Artz, ${ }^{97}$ S. Asai, ${ }^{160}$ N. Asbah, ${ }^{57}$

E. M. Asimakopoulou, ${ }^{169}$ L. Asquith, ${ }^{153}$ K. Assamagan, ${ }^{29}$ R. Astalos, ${ }^{28 a}$ R. J. Atkin, ${ }^{32 \mathrm{a}}$ M. Atkinson, ${ }^{170}$ N. B. Atlay, ${ }^{148}$ K. Augsten, ${ }^{139}$ G. Avolio, ${ }^{35}$ R. Avramidou, ${ }^{58 a}$ M. K. Ayoub, ${ }^{15 a}$ A. M. Azoulay, ${ }^{165 b}$ G. Azuelos, ${ }^{107, f}$ A. E. Baas, ${ }^{59 a}$ M. J. Baca, ${ }^{21}$ H. Bachacou, ${ }^{142}$ K. Bachas, ${ }^{65 a, 65 b}$ M. Backes, ${ }^{132}$ P. Bagnaia,${ }^{70 a, 70 b}$ M. Bahmani,${ }^{82}$ H. Bahrasemani,${ }^{149}$ A. J. Bailey, ${ }^{171}$ V. R. Bailey, ${ }^{170}$ J. T. Baines, ${ }^{141}$ M. Bajic,${ }^{39}$ C. Bakalis, ${ }^{10}$ O. K. Baker, ${ }^{180}$ P. J. Bakker, ${ }^{118}$ D. Bakshi Gupta, ${ }^{8}$ S. Balaji, ${ }^{154}$ E. M. Baldin, ${ }^{120 b, 120 a}$ P. Balek, ${ }^{177}$ F. Balli, ${ }^{142}$ W. K. Balunas, ${ }^{134}$ J. Balz, ${ }^{97}$ E. Banas, ${ }^{82}$ A. Bandyopadhyay,${ }^{24}$ S. Banerjee, ${ }^{178, \mathrm{~g}}$ A. A. E. Bannoura, ${ }^{179}$ L. Barak, ${ }^{158}$ W. M. Barbe, ${ }^{37}$ E. L. Barberio, ${ }^{102}$ D. Barberis,${ }^{53 b, 53 a}$ M. Barbero, ${ }^{99}$ T. Barillari, ${ }^{113}$ M-S. Barisits, ${ }^{35}$ J. Barkeloo, ${ }^{128}$ T. Barklow, ${ }^{150}$ R. Barnea, ${ }^{157}$ S. L. Barnes,${ }^{58 c}$ B. M. Barnett, ${ }^{141}$

R. M. Barnett, ${ }^{18}$ Z. Barnovska-Blenessy, ${ }^{58 a}$ A. Baroncelli, ${ }^{72 a}$ G. Barone,${ }^{29}$ A. J. Barr, ${ }^{132}$ L. Barranco Navarro, ${ }^{171}$ F. Barreiro, ${ }^{96}$ J. Barreiro Guimarães da Costa ${ }^{15 a}$ R. Bartoldus, ${ }^{150}$ A. E. Barton, ${ }^{87}$ P. Bartos, ${ }^{28 a}$ A. Basalaev, ${ }^{135}$ A. Bassalat, ${ }^{129}$ R. L. Bates, ${ }^{55}$ S. J. Batista, ${ }^{164}$ S. Batlamous, ${ }^{34 \mathrm{e}}$ J. R. Batley,${ }^{31}$ M. Battaglia,${ }^{143}$ M. Bauce, ${ }^{70 a, 70 b}$ F. Bauer, ${ }^{142}$ K. T. Bauer, ${ }^{168}$ H. S. Bawa, ${ }^{150, \mathrm{~h}}$ J. B. Beacham, ${ }^{123}$ T. Beau, ${ }^{133}$ P. H. Beauchemin, ${ }^{167}$ P. Bechtle, ${ }^{24}$ H. C. Beck, ${ }^{51}$ H. P. Beck, ${ }^{20, i}$ K. Becker, ${ }^{50}$ M. Becker, ${ }^{97}$ C. Becot, ${ }^{44}$ A. Beddall, ${ }^{12 d}$ A. J. Beddall, ${ }^{12 \mathrm{a}}$ V. A. Bednyakov, ${ }^{77}$ M. Bedognetti, ${ }^{118}$ C. P. Bee, ${ }^{152}$

T. A. Beermann, ${ }^{74}$ M. Begalli, ${ }^{78 b}$ M. Begel, ${ }^{29}$ A. Behera, ${ }^{152}$ J. K. Behr, ${ }^{44}$ F. Beisiegel, ${ }^{24}$ A. S. Bell, ${ }^{92}$ G. Bella, ${ }^{158}$

L. Bellagamba, ${ }^{23 b}$ A. Bellerive, ${ }^{33}$ M. Bellomo, ${ }^{157}$ P. Bellos, ${ }^{9}$ K. Belotskiy, ${ }^{110}$ N. L. Belyaev, ${ }^{110}$ O. Benary, ${ }^{158, a}$ D. Benchekroun, ${ }^{34 a}$ M. Bender, ${ }^{112}$ N. Benekos ${ }^{10}$ Y. Benhammou, ${ }^{158}$ E. Benhar Noccioli, ${ }^{180}$ J. Benitez, ${ }^{75}$ D. P. Benjamin ${ }^{6}$ M. Benoit, ${ }^{52}$ J. R. Bensinger, ${ }^{26}$ S. Bentvelsen, ${ }^{118}$ L. Beresford, ${ }^{132}$ M. Beretta, ${ }^{49}$ D. Berge,${ }^{44}$ E. Bergeaas Kuutmann, ${ }^{169}$ N. Berger, ${ }^{5}$ B. Bergmann, ${ }^{139}$ L. J. Bergsten, ${ }^{26}$ J. Beringer, ${ }^{18}$ S. Berlendis, ${ }^{7}$ N. R. Bernard, ${ }^{100}$ G. Bernardi,${ }^{133}$ C. Bernius, ${ }^{150}$ F. U. Bernlochner, ${ }^{24}$ T. Berry, ${ }^{91}$ P. Berta, ${ }^{97}$ C. Bertella, ${ }^{15 a}$ G. Bertoli, ${ }^{43 a, 43 b}$ I. A. Bertram, ${ }^{87}$ G. J. Besjes, ${ }^{39}$

O. Bessidskaia Bylund, ${ }^{179}$ M. Bessner, ${ }^{44}$ N. Besson, ${ }^{142}$ A. Bethani, ${ }^{98}$ S. Bethke, ${ }^{113}$ A. Betti, ${ }^{24}$ A. J. Bevan, ${ }^{90}$ J. Beyer, ${ }^{113}$ R. Bi, ${ }^{136}$ R. M. Bianchi, ${ }^{136}$ O. Biebel, ${ }^{112}$ D. Biedermann, ${ }^{19}$ R. Bielski,${ }^{35}$ K. Bierwagen, ${ }^{97}$ N. V. Biesuz, ${ }^{69 a, 69 b}$ M. Biglietti, ${ }^{72 a}$ T. R. V. Billoud, ${ }^{107}$ M. Bindi, ${ }^{51}$ A. Bingul, ${ }^{12 d}$ C. Bini, ${ }^{70 a, 70 b}$ S. Biondi, ${ }^{23 b, 23 a}$ M. Birman, ${ }^{177}$ T. Bisanz, ${ }^{51}$ J. P. Biswal, ${ }^{158}$ C. Bittrich, ${ }^{46}$ D. M. Bjergaard, ${ }^{47}$ J. E. Black, ${ }^{150}$ K. M. Black,${ }^{25}$ T. Blazek, ${ }^{28 a}$ I. Bloch,${ }^{44}$ C. Blocker ${ }^{26}$ A. Blue,${ }^{55}$ U. Blumenschein, ${ }^{90}$ S. Blunier, ${ }^{144 a}$ G. J. Bobbink, ${ }^{118}$ V. S. Bobrovnikov, ${ }^{120 b, 120 a}$ S. S. Bocchetta, ${ }^{94}$ A. Bocci, ${ }^{47}$ D. Boerner, ${ }^{179}$ D. Bogavac, ${ }^{112}$ A. G. Bogdanchikov, ${ }^{120 b, 120 a}$ C. Bohm, ${ }^{43 a}$ V. Boisvert, ${ }^{91}$ P. Bokan, ${ }^{169}$ T. Bold ${ }^{81 a}$ A. S. Boldyrev, ${ }^{111}$ A. E. Bolz ${ }^{59 b}$ M. Bomben, ${ }^{133}$ M. Bona, ${ }^{90}$ J. S. Bonilla, ${ }^{128}$ M. Boonekamp,${ }^{142}$ H. M. Borecka-Bielska, ${ }^{88}$ A. Borisov, ${ }^{121}$ G. Borissov, ${ }^{87}$ J. Bortfeldt, ${ }^{35}$ D. Bortoletto, ${ }^{132}$ V. Bortolotto,${ }^{71 a, 71 b}$ D. Boscherini, ${ }^{23 b}$ M. Bosman, ${ }^{14}$ J. D. Bossio Sola,${ }^{30}$ K. Bouaouda, ${ }^{34 a}$ J. Boudreau, ${ }^{136}$ E. V. Bouhova-Thacker, ${ }^{87}$ D. Boumediene, ${ }^{37}$ C. Bourdarios, ${ }^{129}$ S. K. Boutle, ${ }^{55}$ A. Boveia, ${ }^{123}$ J. Boyd ${ }^{35}$ D. Boye, ${ }^{32 b}$ I. R. Boyko, ${ }^{77}$ A. J. Bozson, ${ }^{91}$ J. Bracinik, ${ }^{21}$ N. Brahimi, ${ }^{99}$ A. Brandt,${ }^{8}$ G. Brandt, ${ }^{179}$ O. Brandt ${ }^{59 a}$ F. Braren, ${ }^{44}$ U. Bratzler, ${ }^{161}$ B. Brau, ${ }^{100}$ J. E. Brau, ${ }^{128}$ W. D. Breaden Madden, ${ }^{55}$ K. Brendlinger, ${ }^{44}$ L. Brenner, ${ }^{44}$ R. Brenner, ${ }^{169}$ S. Bressler, ${ }^{177}$ B. Brickwedde, ${ }^{97}$ D. L. Briglin, ${ }^{21}$ D. Britton,${ }^{55}$ D. Britzger, ${ }^{113}$ I. Brock, ${ }^{24}$ R. Brock, ${ }^{104}$ G. Brooijmans, ${ }^{38}$ T. Brooks, ${ }^{91}$ W. K. Brooks, ${ }^{144 \mathrm{~b}}$ E. Brost, ${ }^{119}$ J. H Broughton, ${ }^{21}$ P. A. Bruckman de Renstrom, ${ }^{82}$ D. Bruncko, ${ }^{28 b}$ A. Bruni, ${ }^{23 b}$ G. Bruni, ${ }^{23 b}$ L. S. Bruni, ${ }^{118}$ S. Bruno, ${ }^{71 a, 71 b}$ B. H. Brunt, ${ }^{31}$ M. Bruschi, ${ }^{23 b}$ N. Bruscino, ${ }^{136}$ P. Bryant, ${ }^{36}$ L. Bryngemark, ${ }^{94}$ T. Buanes,${ }^{17}$ Q. Buat,${ }^{35}$ P. Buchholz,${ }^{148}$ A. G. Buckley, ${ }^{55}$ I. A. Budagov, ${ }^{77}$ M. K. Bugge,${ }^{131}$ F. Bührer, ${ }^{50}$ O. Bulekov, ${ }^{110}$ D. Bullock, ${ }^{8}$ T. J. Burch, ${ }^{119}$ S. Burdin, ${ }^{88}$ C. D. Burgard ${ }^{118}$ A. M. Burger, ${ }^{5}$ B. Burghgrave, ${ }^{119}$ K. Burka ${ }^{82}$ S. Burke, ${ }^{141}$ I. Burmeister, ${ }^{45}$ J. T. P. Burr, ${ }^{132}$ V. Büscher, ${ }^{97}$ E. Buschmann, ${ }^{51}$ P. Bussey,${ }^{55}$ J. M. Butler, ${ }^{25}$ C. M. Buttar, ${ }^{55}$ J. M. Butterworth, ${ }^{92}$ P. Butti, ${ }^{35}$ W. Buttinger, ${ }^{35}$ A. Buzatu, ${ }^{155}$ A. R. Buzykaev, ${ }^{120 b, 120 a}$ G. Cabras, ${ }^{23 b, 23 a}$ S. Cabrera Urbán, ${ }^{171}$ D. Caforio, ${ }^{139}$ H. Cai, ${ }^{170}$ V. M. M. Cairo, ${ }^{2}$ O. Cakir, ${ }^{4 a}$ N. Calace, ${ }^{52}$ P. Calafiura, ${ }^{18}$ A. Calandri, ${ }^{99}$ G. Calderini, ${ }^{133}$ P. Calfayan, ${ }^{63}$ G. Callea, ${ }^{55}$ L. P. Caloba, ${ }^{78 b}$ S. Calvente Lopez, ${ }^{96}$ D. Calvet, ${ }^{37}$ S. Calvet, ${ }^{37}$ T. P. Calvet, ${ }^{152}$ M. Calvetti, ${ }^{69 a, 69 b}$ R. Camacho Toro, ${ }^{133}$ S. Camarda, ${ }^{35}$ D. Camarero Munoz, ${ }^{96}$ P. Camarri, ${ }^{71 a, 71 b}$ D. Cameron, ${ }^{131}$ R. Caminal Armadans, ${ }^{100}$ C. Camincher, ${ }^{35}$ S. Campana, ${ }^{35}$ M. Campanelli, ${ }^{92}$ A. Camplani, ${ }^{39}$ A. Campoverde, ${ }^{148}$ V. Canale, ${ }^{67 a, 67 b}$ M. Cano Bret,${ }^{58 \mathrm{c}}$ J. Cantero, ${ }^{126}$ T. Cao, ${ }^{158}$ Y. Cao, ${ }^{170}$ M. D. M. Capeans Garrido, ${ }^{35}$ I. Caprini, ${ }^{27 b}$ 
M. Caprini, ${ }^{27 b}$ M. Capua, ${ }^{40 b, 40 a}$ R. M. Carbone, ${ }^{38}$ R. Cardarelli, ${ }^{71 a}$ F. C. Cardillo, ${ }^{146}$ I. Carli, ${ }^{140}$ T. Carli, ${ }^{35}$ G. Carlino, ${ }^{67 a}$ B. T. Carlson, ${ }^{136}$ L. Carminati, ${ }^{66 a, 66 b}$ R. M. D. Carney, ${ }^{43 a, 43 b}$ S. Caron, ${ }^{117}$ E. Carquin, ${ }^{144 b}$ S. Carrá, ${ }^{66 a, 66 b}$ J. W. S. Carter, ${ }^{164}$ D. Casadei, ${ }^{32 \mathrm{~b}}$ M. P. Casado, ${ }^{14, j}$ A. F. Casha, ${ }^{164}$ D. W. Casper, ${ }^{168}$ R. Castelijn, ${ }^{118}$ F. L. Castillo, ${ }^{171}$ V. Castillo Gimenez, ${ }^{171}$ N. F. Castro, ${ }^{137 a, 137 e}$ A. Catinaccio, ${ }^{35}$ J. R. Catmore, ${ }^{131}$ A. Cattai,${ }^{35}$ J. Caudron,${ }^{24}$ V. Cavaliere,${ }^{29}$ E. Cavallaro, ${ }^{14}$ D. Cavalli, ${ }^{66 a}$ M. Cavalli-Sforza, ${ }^{14}$ V. Cavasinni, ${ }^{69 a, 69 b}$ E. Celebi, ${ }^{12 b}$ F. Ceradini,${ }^{72 a, 72 b}$ L. Cerda Alberich, ${ }^{171}$ A. S. Cerqueira, ${ }^{78 a}$ A. Cerri, ${ }^{153}$ L. Cerrito, ${ }^{71 a, 71 b}$ F. Cerutti, ${ }^{18}$ A. Cervelli, ${ }^{23 b, 23 a}$ S. A. Cetin, ${ }^{12 b}$ A. Chafaq, ${ }^{34 a}$ D. Chakraborty, ${ }^{119}$ S. K. Chan, ${ }^{57}$ W. S. Chan, ${ }^{118}$ J. D. Chapman, ${ }^{31}$ B. Chargeishvili, ${ }^{156 b}$ D. G. Charlton, ${ }^{21}$ C. C. Chau, ${ }^{33}$ C. A. Chavez Barajas, ${ }^{153}$ S. Che, ${ }^{123}$

A. Chegwidden, ${ }^{104}$ S. Chekanov, ${ }^{6}$ S. V. Chekulaev, ${ }^{165 a}$ G. A. Chelkov, ${ }^{77, k}$ M. A. Chelstowska, ${ }^{35}$ C. Chen,${ }^{58 a}$ C. H. Chen, ${ }^{76}$ H. Chen, ${ }^{29}$ J. Chen, ${ }^{58 a}$ J. Chen, ${ }^{38}$ S. Chen, ${ }^{134}$ S. J. Chen, ${ }^{15 c}$ X. Chen, ${ }^{15 b, 1}$ Y. Chen, ${ }^{80}$ Y-H. Chen, ${ }^{44}$ H. C. Cheng, ${ }^{61 a}$

H. J. Cheng, ${ }^{15 \mathrm{~d}}$ A. Cheplakov, ${ }^{77}$ E. Cheremushkina, ${ }^{121}$ R. Cherkaoui El Moursli, ${ }^{34 \mathrm{e}}$ E. Cheu, ${ }^{7}$ K. Cheung, ${ }^{62}$

T. J. A. Chevalérias, ${ }^{142}$ L. Chevalier, ${ }^{142}$ V. Chiarella, ${ }^{49}$ G. Chiarelli, ${ }^{69 a}$ G. Chiodini, ${ }^{65 a}$ A. S. Chisholm, ${ }^{35,21}$ A. Chitan, ${ }^{27 b}$

I. Chiu, ${ }^{160}$ Y. H. Chiu, ${ }^{173}$ M. V. Chizhov, ${ }^{77}$ K. Choi, ${ }^{63}$ A. R. Chomont, ${ }^{129}$ S. Chouridou, ${ }^{159}$ Y. S. Chow, ${ }^{118}$

V. Christodoulou, ${ }^{92}$ M. C. Chu, ${ }^{61 a}$ J. Chudoba, ${ }^{138}$ A. J. Chuinard, ${ }^{101}$ J. J. Chwastowski, ${ }^{82}$ L. Chytka, ${ }^{127}$ D. Cinca, ${ }^{45}$ V. Cindro, ${ }^{89}$ I. A. Cioară, ${ }^{24}$ A. Ciocio, ${ }^{18}$ F. Cirotto, ${ }^{67 a, 67 b}$ Z. H. Citron, ${ }^{177}$ M. Citterio, ${ }^{66 a}$ A. Clark, ${ }^{52}$ M. R. Clark, ${ }^{38}$

P. J. Clark, ${ }^{48}$ C. Clement, ${ }^{43 a, 43 b}$ Y. Coadou, ${ }^{99}$ M. Cobal,${ }^{64 a, 64 c}$ A. Coccaro, ${ }^{53 b, 53 a}$ J. Cochran,${ }^{76}$ H. Cohen, ${ }^{158}$ A. E. C. Coimbra, ${ }^{177}$ L. Colasurdo, ${ }^{117}$ B. Cole, ${ }^{38}$ A. P. Colijn, ${ }^{118}$ J. Collot, ${ }^{56}$ P. Conde Muiño, ${ }^{137 a, m}$ E. Coniavitis,${ }^{50}$ S. H. Connell, ${ }^{32 b}$ I. A. Connelly, ${ }^{98}$ S. Constantinescu, ${ }^{27 b}$ F. Conventi, ${ }^{67 a, n}$ A. M. Cooper-Sarkar, ${ }^{132}$ F. Cormier, ${ }^{172}$ K. J. R. Cormier, ${ }^{164}$ L. D. Corpe, ${ }^{92}$ M. Corradi, ${ }^{70 a, 70 b}$ E. E. Corrigan, ${ }^{94}$ F. Corriveau, ${ }^{101,0}$ A. Cortes-Gonzalez, ${ }^{35}$ M. J. Costa, ${ }^{171}$ F. Costanza, ${ }^{5}$ D. Costanzo, ${ }^{146}$ G. Cottin, ${ }^{31}$ G. Cowan, ${ }^{91}$ B. E. Cox, ${ }^{98}$ J. Crane, ${ }^{98}$ K. Cranmer, ${ }^{122}$ S. J. Crawley, ${ }^{55}$ R. A. Creager, ${ }^{134}$ G. Cree,${ }^{33}$ S. Crépé-Renaudin, ${ }^{56}$ F. Crescioli ${ }^{133}$ M. Cristinziani, ${ }^{24}$ V. Croft, ${ }^{122}$ G. Crosetti, ${ }^{40 b, 40 a}$ A. Cueto, ${ }^{96}$ T. Cuhadar Donszelmann, ${ }^{146}$ A. R. Cukierman, ${ }^{150}$ S. Czekierda, ${ }^{82}$ P. Czodrowski, ${ }^{35}$ M. J. Da Cunha Sargedas De Sousa, ${ }^{58 \mathrm{~b}}$ C. Da Via ${ }^{98}$ W. Dabrowski, ${ }^{81 \mathrm{a}}$ T. Dado, ${ }^{28 \mathrm{a}, \mathrm{p}}$ S. Dahbi, ${ }^{34 \mathrm{e}}$ T. Dai,${ }^{103}$ F. Dallaire, ${ }^{107}$ C. Dallapiccola ${ }^{100}$ M. Dam ${ }^{39}$ G. D'amen ${ }^{23 b, 23 a}$ J. Damp,${ }^{97}$ J. R. Dandoy, ${ }^{134}$ M. F. Daneri, ${ }^{30}$ N. P. Dang, ${ }^{178, g}$ N. D Dann,${ }^{98}$ M. Danninger, ${ }^{172}$ V. Dao, ${ }^{35}$ G. Darbo,${ }^{53 \mathrm{~b}}$ S. Darmora, ${ }^{8}$ O. Dartsi, ${ }^{5}$ A. Dattagupta, ${ }^{128}$ T. Daubney, ${ }^{44}$ S. D'Auria, ${ }^{66 a, 66 b}$ W. Davey, ${ }^{24}$ C. David, ${ }^{44}$ T. Davidek, ${ }^{140}$ D. R. Davis, ${ }^{47}$ E. Dawe, ${ }^{102}$ I. Dawson, ${ }^{146}$ K. De,${ }^{8}$ R. De Asmundis,${ }^{67 a}$ A. De Benedetti, ${ }^{125}$ M. De Beurs, ${ }^{118}$ S. De Castro, ${ }^{23 b, 23 a}$ S. De Cecco, ${ }^{70 a, 70 b}$ N. De Groot, ${ }^{117}$ P. de Jong, ${ }^{118}$ H. De la Torre, ${ }^{104}$ F. De Lorenzi ${ }^{76}$ A. De Maria, ${ }^{69 a, 69 b}$ D. De Pedis, ${ }^{70 a}$ A. De Salvo, ${ }^{70 a}$ U. De Sanctis,${ }^{71 a, 71 b}$ M. De Santis, ${ }^{71 a, 71 b}$ A. De Santo, ${ }^{153}$

K. De Vasconcelos Corga, ${ }^{99}$ J. B. De Vivie De Regie, ${ }^{129}$ C. Debenedetti, ${ }^{143}$ D. V. Dedovich, ${ }^{77}$ N. Dehghanian, ${ }^{3}$ M. Del Gaudio, ${ }^{40 b, 40 a}$ J. Del Peso, ${ }^{96}$ Y. Delabat Diaz, ${ }^{44}$ D. Delgove, ${ }^{129}$ F. Deliot,${ }^{142}$ C. M. Delitzsch, ${ }^{7}$ M. Della Pietra,${ }^{67 a, 67 b}$ D. Della Volpe ${ }^{52}$ A. Dell'Acqua, ${ }^{35}$ L. Dell'Asta ${ }^{25}$ M. Delmastro, ${ }^{5}$ C. Delporte, ${ }^{129}$ P. A. Delsart, ${ }^{56}$ D. A. DeMarco, ${ }^{164}$ S. Demers ${ }^{180}$ M. Demichev, ${ }^{77}$ S. P. Denisov, ${ }^{121}$ D. Denysiuk, ${ }^{118}$ L. D’Eramo, ${ }^{133}$ D. Derendarz, ${ }^{82}$ J. E. Derkaoui, ${ }^{34 d}$ F. Derue, ${ }^{133}$ P. Dervan, ${ }^{88}$ K. Desch, ${ }^{24}$ C. Deterre,${ }^{44}$ K. Dette,${ }^{164}$ M. R. Devesa, ${ }^{30}$ P. O. Deviveiros,${ }^{35}$ A. Dewhurst ${ }^{141}$ S. Dhaliwal, ${ }^{26}$ F. A. Di Bello, ${ }^{52}$ A. Di Ciaccio, ${ }^{71 a, 71 b}$ L. Di Ciaccio,${ }^{5}$ W. K. Di Clemente, ${ }^{134}$ C. Di Donato,${ }^{67,67 b}$ A. Di Girolamo, ${ }^{35}$ G. Di Gregorio,${ }^{69 a, 69 b}$ B. Di Micco, ${ }^{72 a, 72 b}$ R. Di Nardo, ${ }^{100}$ K. F. Di Petrillo, ${ }^{57}$ R. Di Sipio, ${ }^{164}$ D. Di Valentino, ${ }^{33}$ C. Diaconu, ${ }^{99}$ M. Diamond, ${ }^{164}$ F. A. Dias, ${ }^{39}$ T. Dias Do Vale, ${ }^{137 a}$ M. A. Diaz, ${ }^{144 a}$ J. Dickinson, ${ }^{18}$ E. B. Diehl, ${ }^{103}$ J. Dietrich, ${ }^{19}$ S. Díez Cornell, ${ }^{44}$ A. Dimitrievska, ${ }^{18}$ J. Dingfelder, ${ }^{24}$ F. Dittus, ${ }^{35}$ F. Djama, ${ }^{99}$ T. Djobava, ${ }^{156 b}$ J. I. Djuvsland, ${ }^{59 \mathrm{a}}$ M. A. B. Do Vale, ${ }^{78 \mathrm{c}}$ M. Dobre, ${ }^{27 \mathrm{~b}}$ D. Dodsworth, ${ }^{26}$ C. Doglioni, ${ }^{94}$ J. Dolejsi, ${ }^{140}$ Z. Dolezal, ${ }^{140}$ M. Donadelli, ${ }^{78 d}$ J. Donini, ${ }^{37}$ A. D'onofrio, ${ }^{90}$ M. D'Onofrio ${ }^{88}$ J. Dopke, ${ }^{141}$ A. Doria, ${ }^{67 a}$ M. T. Dova, ${ }^{86}$ A. T. Doyle,${ }^{55}$ E. Drechsler, ${ }^{51}$ E. Dreyer, ${ }^{149}$ T. Dreyer, ${ }^{51}$ Y. Du, ${ }^{58 b}$ F. Dubinin, ${ }^{108}$ M. Dubovsky, ${ }^{28 a}$ A. Dubreuil,${ }^{52}$ E. Duchovni, ${ }^{177}$ G. Duckeck, ${ }^{112}$ A. Ducourthial, ${ }^{133}$ O. A. Ducu, ${ }^{107, q}$ D. Duda, ${ }^{113}$ A. Dudarev, ${ }^{35}$ A. C. Dudder, ${ }^{97}$ E. M. Duffield, ${ }^{18}$ L. Duflot, ${ }^{129}$ M. Dührssen, ${ }^{35}$ C. Dülsen, ${ }^{179}$ M. Dumancic, ${ }^{177}$ A. E. Dumitriu, ${ }^{27 b, r}$ A. K. Duncan, ${ }^{55}$ M. Dunford, ${ }^{59 a}$ A. Duperrin, ${ }^{99}$ H. Duran Yildiz, ${ }^{4 a}$ M. Düren,${ }^{54}$ A. Durglishvili, ${ }^{156 b}$ D. Duschinger, ${ }^{46}$ B. Dutta, ${ }^{44}$ D. Duvnjak, ${ }^{1}$ M. Dyndal,${ }^{44}$ S. Dysch ${ }^{98}$ B. S. Dziedzic, ${ }^{82}$ C. Eckardt, ${ }^{44}$ K. M. Ecker, ${ }^{113}$ R. C. Edgar, ${ }^{103}$ T. Eifert, ${ }^{35}$ G. Eigen, ${ }^{17}$ K. Einsweiler, ${ }^{18}$ T. Ekelof, ${ }^{169}$ M. El Kacimi, ${ }^{34 c}$ R. El Kosseifi, ${ }^{99}$ V. Ellajosyula, ${ }^{99}$ M. Ellert, ${ }^{169}$ F. Ellinghaus, ${ }^{179}$ A. A. Elliot,${ }^{90}$ N. Ellis, ${ }^{35}$ J. Elmsheuser, ${ }^{29}$ M. Elsing, ${ }^{35}$ D. Emeliyanov, ${ }^{141}$ A. Emerman, ${ }^{38}$ Y. Enari, ${ }^{160}$ J. S. Ennis, ${ }^{175}$ M. B. Epland, ${ }^{47}$ J. Erdmann, ${ }^{45}$ A. Ereditato, ${ }^{20}$ S. Errede,${ }^{170}$ M. Escalier, ${ }^{129}$ C. Escobar, ${ }^{171}$ O. Estrada Pastor, ${ }^{171}$ A. I. Etienvre, ${ }^{142}$ E. Etzion, ${ }^{158}$ H. Evans,${ }^{63}$ A. Ezhilov, ${ }^{135}$ M. Ezzi ${ }^{34 \mathrm{e}}$ F. Fabbri, ${ }^{55}$ L. Fabbri, ${ }^{23 b, 23 a}$ V. Fabiani, ${ }^{117}$ G. Facini, ${ }^{92}$ R. M. Faisca Rodrigues Pereira, ${ }^{137 a}$

R. M. Fakhrutdinov, ${ }^{121}$ S. Falciano, ${ }^{70 a}$ P. J. Falke, ${ }^{5}$ S. Falke, ${ }^{5}$ J. Faltova, ${ }^{140}$ Y. Fang, ${ }^{15 a}$ M. Fanti, ${ }^{66,66 b}$ A. Farbin, ${ }^{8}$ A. Farilla, ${ }^{72 a}$ E. M. Farina, ${ }^{68 a, 68 b}$ T. Farooque, ${ }^{104}$ S. Farrell, ${ }^{18}$ S. M. Farrington, ${ }^{175}$ P. Farthouat, ${ }^{35}$ F. Fassi, ${ }^{34 \mathrm{e}}$ P. Fassnacht,${ }^{35}$ 
D. Fassouliotis, ${ }^{9}$ M. Faucci Giannelli, ${ }^{48}$ A. Favareto, ${ }^{53 b, 53 a}$ W. J. Fawcett, ${ }^{31}$ L. Fayard,${ }^{129}$ O. L. Fedin, ${ }^{135, s}$ W. Fedorko, ${ }^{172}$ M. Feickert, ${ }^{41}$ S. Feigl,${ }^{131}$ L. Feligioni, ${ }^{99}$ C. Feng,${ }^{58 b}$ E. J. Feng,${ }^{35}$ M. Feng, ${ }^{47}$ M. J. Fenton, ${ }^{55}$ A. B. Fenyuk,${ }^{121}$ L. Feremenga, ${ }^{8}$ J. Ferrando, ${ }^{44}$ A. Ferrari, ${ }^{169}$ P. Ferrari, ${ }^{118}$ R. Ferrari, ${ }^{68 a}$ D. E. Ferreira de Lima, ${ }^{59 b}$ A. Ferrer, ${ }^{171}$ D. Ferrere, ${ }^{52}$ C. Ferretti, ${ }^{103}$ F. Fiedler, ${ }^{97}$ A. Filipčič,${ }^{89}$ F. Filthaut, ${ }^{117}$ K. D. Finelli, ${ }^{25}$ M. C. N. Fiolhais, ${ }^{137 a, 137 c, t}$ L. Fiorini, ${ }^{171}$ C. Fischer,${ }^{14}$ W. C. Fisher, ${ }^{104}$ N. Flaschel,${ }^{44}$ I. Fleck, ${ }^{148}$ P. Fleischmann, ${ }^{103}$ R. R. M. Fletcher, ${ }^{134}$ T. Flick, ${ }^{179}$ B. M. Flierl, ${ }^{112}$ L. M. Flores, ${ }^{134}$ L. R. Flores Castillo, ${ }^{61 a}$ F. M. Follega,${ }^{73 a, 73 b}$ N. Fomin, ${ }^{17}$ G. T. Forcolin, ${ }^{73 a, 73 b}$ A. Formica, ${ }^{142}$ F. A. Förster, ${ }^{14}$ A. C. Forti, ${ }^{98}$ A. G. Foster, ${ }^{21}$ D. Fournier, ${ }^{129}$ H. Fox,${ }^{87}$ S. Fracchia, ${ }^{146}$ P. Francavilla, ${ }^{69 a, 69 b}$ M. Franchini, ${ }^{23 b, 23 a}$ S. Franchino, ${ }^{59 \mathrm{a}}$ D. Francis, ${ }^{35}$ L. Franconi, ${ }^{143}$ M. Franklin, ${ }^{57}$ M. Frate, ${ }^{168}$ M. Fraternali, ${ }^{68 a, 68 b}$ A. N. Fray, ${ }^{90}$ D. Freeborn,${ }^{92}$ B. Freund, ${ }^{107}$ W. S. Freund, ${ }^{78 b}$ E. M. Freundlich, ${ }^{45}$ D. C. Frizzell, ${ }^{125}$ D. Froidevaux,${ }^{35}$ J. A. Frost,${ }^{132}$ C. Fukunaga, ${ }^{161}$ E. Fullana Torregrosa, ${ }^{171}$ T. Fusayasu, ${ }^{114}$ J. Fuster, ${ }^{171}$ O. Gabizon, ${ }^{157}$ A. Gabrielli, ${ }^{23 b, 23 a}$ A. Gabrielli, ${ }^{18}$ G. P. Gach,${ }^{81 a}$ S. Gadatsch, ${ }^{52}$ P. Gadow, ${ }^{113}$ G. Gagliardi, ${ }^{53 b, 53 a}$ L. G. Gagnon, ${ }^{107}$ C. Galea, ${ }^{27 b}$ B. Galhardo, ${ }^{137 a, 137 \mathrm{c}}$ E. J. Gallas, ${ }^{132}$ B. J. Gallop, ${ }^{141}$ P. Gallus,${ }^{139}$ G. Galster ${ }^{39}$ R. Gamboa Goni,${ }^{90}$ K. K. Gan,${ }^{123}$ S. Ganguly, ${ }^{177}$ J. Gao, ${ }^{58 a}$ Y. Gao,${ }^{88}$ Y. S. Gao, ${ }^{150, h}$ C. García, ${ }^{171}$ J. E. García Navarro, ${ }^{171}$ J. A. García Pascual, ${ }^{15 a}$ M. Garcia-Sciveres, ${ }^{18}$ R. W. Gardner, ${ }^{36}$ N. Garelli, ${ }^{150}$ S. Gargiulo ${ }^{50}$ V. Garonne, ${ }^{131}$ K. Gasnikova, ${ }^{44}$ A. Gaudiello, ${ }^{53 b, 53 a}$ G. Gaudio, ${ }^{68 a}$ I. L. Gavrilenko, ${ }^{108}$ A. Gavrilyuk, ${ }^{109}$ C. Gay, ${ }^{172}$ G. Gaycken ${ }^{24}$ E. N. Gazis, ${ }^{10}$ C. N. P. Gee,${ }^{141}$ J. Geisen,${ }^{51}$ M. Geisen, ${ }^{97}$ M. P. Geisler,${ }^{59 a}$ C. Gemme, ${ }^{53 b}$ M. H. Genest, ${ }^{56}$ C. Geng, ${ }^{103}$ S. Gentile, ${ }^{70,70 b}$ S. George, ${ }^{91}$ D. Gerbaudo, ${ }^{14}$ G. Gessner,${ }^{45}$ S. Ghasemi, ${ }^{148}$ M. Ghasemi Bostanabad, ${ }^{173}$ M. Ghneimat, ${ }^{24}$ B. Giacobbe, ${ }^{23 b}$ S. Giagu, ${ }^{70 a, 70 b}$ N. Giangiacomi ${ }^{23 b, 23 a}$ P. Giannetti, ${ }^{69 a}$ A. Giannini, ${ }^{67 a, 67 b}$ S. M. Gibson, ${ }^{91}$ M. Gignac, ${ }^{143}$ D. Gillberg, ${ }^{33}$ G. Gilles, ${ }^{179}$ D. M. Gingrich, ${ }^{3, f}$ M. P. Giordani, ${ }^{64 a, 64 c}$ F. M. Giorgi, ${ }^{23 b}$ P. F. Giraud ${ }^{142}$ P. Giromini, ${ }^{57}$ G. Giugliarelli, ${ }^{64,64 c}$ D. Giugni, ${ }^{66 a}$ F. Giuli, ${ }^{132}$ M. Giulini, ${ }^{59 b}$ S. Gkaitatzis, ${ }^{159}$ I. Gkialas, ${ }^{9, u}$ E. L. Gkougkousis, ${ }^{14}$ P. Gkountoumis,${ }^{10}$ L. K. Gladilin, ${ }^{111}$ C. Glasman, ${ }^{96}$ J. Glatzer ${ }^{14}$ P. C. F. Glaysher, ${ }^{44}$ A. Glazov, ${ }^{44}$ M. Goblirsch-Kolb, ${ }^{26}$ J. Godlewski, ${ }^{82}$ S. Goldfarb, ${ }^{102}$ T. Golling, ${ }^{52}$ D. Golubkov, ${ }^{121}$ A. Gomes, ${ }^{137 a, 137 b}$ R. Goncalves Gama, ${ }^{51}$ R. Gonçalo, ${ }^{137 a}$ G. Gonella, ${ }^{50}$ L. Gonella, ${ }^{21}$ A. Gongadze, ${ }^{77}$ F. Gonnella, ${ }^{21}$ J. L. Gonski, ${ }^{57}$ S. González de la Hoz, ${ }^{171}$ S. Gonzalez-Sevilla, ${ }^{52}$ L. Goossens,${ }^{35}$ P. A. Gorbounov, ${ }^{109}$ H. A. Gordon, ${ }^{29}$ B. Gorini ${ }^{35}$ E. Gorini, ${ }^{65 a, 65 b}$ A. Gorišek, ${ }^{89}$ A. T. Goshaw, ${ }^{47}$ C. Gössling,${ }^{45}$ M. I. Gostkin, ${ }^{77}$ C. A. Gottardo, ${ }^{24}$ C. R. Goudet, ${ }^{129}$ D. Goujdami, ${ }^{34 \mathrm{c}}$ A. G. Goussiou, ${ }^{145}$ N. Govender, ${ }^{32 b, v}$ C. Goy, ${ }^{5}$ E. Gozani, ${ }^{157}$ I. Grabowska-Bold, ${ }^{81 a}$ P. O. J. Gradin, ${ }^{169}$ E. C. Graham, ${ }^{88}$ J. Gramling, ${ }^{168}$ E. Gramstad, ${ }^{131}$ S. Grancagnolo, ${ }^{19}$ V. Gratchev, ${ }^{135}$ P. M. Gravila, ${ }^{27 f}$ F. G. Gravili, ${ }^{65 a, 65 b}$ C. Gray, ${ }^{55}$ H. M. Gray, ${ }^{18}$ Z. D. Greenwood, ${ }^{93, w}$ C. Grefe, ${ }^{24}$ K. Gregersen, ${ }^{94}$ I. M. Gregor,${ }^{44}$ P. Grenier, ${ }^{150}$ K. Grevtsov, ${ }^{44}$ N. A. Grieser, ${ }^{125}$ J. Griffiths, ${ }^{8}$ A. A. Grillo, ${ }^{143}$ K. Grimm, ${ }^{150, x}$ S. Grinstein, ${ }^{14, y}$ Ph. Gris, ${ }^{37}$ J.-F. Grivaz, ${ }^{129}$ S. Groh, ${ }^{97}$ E. Gross, ${ }^{177}$ J. Grosse-Knetter, ${ }^{51}$ G. C. Grossi, ${ }^{93}$ Z. J. Grout,${ }^{92}$ C. Grud, ${ }^{103}$ A. Grummer, ${ }^{116}$ L. Guan, ${ }^{103}$ W. Guan, ${ }^{178}$ J. Guenther, ${ }^{35}$ A. Guerguichon, ${ }^{129}$ F. Guescini, ${ }^{165 a}$ D. Guest,${ }^{168}$ R. Gugel,${ }^{50}$ B. Gui, ${ }^{123}$ T. Guillemin, ${ }^{5}$ S. Guindon, ${ }^{35}$ U. Gul ${ }^{55}$ C. Gumpert, ${ }^{35}$ J. Guo,${ }^{5 \mathrm{c}}$ W. Guo, ${ }^{103}$ Y. Guo, ${ }^{58, \mathrm{a}} \mathrm{Z}$. Guo, ${ }^{99}$ R. Gupta, ${ }^{44}$ S. Gurbuz, ${ }^{12 \mathrm{c}}$ G. Gustavino, ${ }^{125}$ B. J. Gutelman, ${ }^{157}$ P. Gutierrez, ${ }^{125}$ C. Gutschow, ${ }^{92}$ C. Guyot, ${ }^{142}$ M. P. Guzik, ${ }^{81 a}$ C. Gwenlan, ${ }^{132}$ C. B. Gwilliam, ${ }^{88}$ A. Haas, ${ }^{122}$ C. Haber, ${ }^{18}$ H. K. Hadavand, ${ }^{8}$ N. Haddad, ${ }^{34 \mathrm{e}}$ A. Hadef,${ }^{58 \mathrm{a}}$ S. Hageböck, ${ }^{24}$ M. Hagihara, ${ }^{166}$ H. Hakobyan, ${ }^{181, \mathrm{a}}$ M. Haleem, ${ }^{174}$ J. Haley, ${ }^{126}$

G. Halladjian, ${ }^{104}$ G. D. Hallewell, ${ }^{99}$ K. Hamacher, ${ }^{179}$ P. Hamal, ${ }^{127}$ K. Hamano, ${ }^{173}$ A. Hamilton, ${ }^{32 a}$ G. N. Hamity, ${ }^{146}$ K. Han,${ }^{58 a \text { aa }}$ L. Han,${ }^{58 a}$ S. Han,${ }^{15 d}$ K. Hanagaki, ${ }^{79, b b}$ M. Hance,${ }^{143}$ D. M. Handl, ${ }^{112}$ B. Haney, ${ }^{134}$ R. Hankache,${ }^{133}$ P. Hanke, ${ }^{59 a}$ E. Hansen, ${ }^{94}$ J. B. Hansen, ${ }^{39}$ J. D. Hansen, ${ }^{39}$ M. C. Hansen, ${ }^{24}$ P. H. Hansen, ${ }^{39}$ K. Hara, ${ }^{166}$ A. S. Hard, ${ }^{178}$ T. Harenberg, ${ }^{179}$ S. Harkusha, ${ }^{105}$ P. F. Harrison, ${ }^{175}$ N. M. Hartmann, ${ }^{112}$ Y. Hasegawa, ${ }^{147}$ A. Hasib ${ }^{48}$ S. Hassani, ${ }^{142}$ S. Haug, ${ }^{20}$ R. Hauser, ${ }^{104}$

L. Hauswald, ${ }^{46}$ L. B. Havener, ${ }^{38}$ M. Havranek, ${ }^{139}$ C. M. Hawkes, ${ }^{21}$ R. J. Hawkings, ${ }^{35}$ D. Hayden, ${ }^{104}$ C. Hayes ${ }^{152}$ C. P. Hays, ${ }^{132}$ J. M. Hays, ${ }^{90}$ H. S. Hayward,${ }^{88}$ S. J. Haywood, ${ }^{141}$ F. He, ${ }^{58 a}$ M. P. Heath,${ }^{48}$ V. Hedberg, ${ }^{94}$ L. Heelan, ${ }^{8}$ S. Heer, ${ }^{24}$ K. K. Heidegger, ${ }^{50}$ J. Heilman, ${ }^{33}$ S. Heim, ${ }^{44}$ T. Heim, ${ }^{18}$ B. Heinemann, ${ }^{44, c c}$ J. J. Heinrich, ${ }^{112}$ L. Heinrich, ${ }^{122}$ C. Heinz, ${ }^{54}$ J. Hejbal, ${ }^{138}$ L. Helary, ${ }^{35}$ A. Held, ${ }^{172}$ S. Hellesund, ${ }^{131}$ C. M. Helling ${ }^{143}$ S. Hellman, ${ }^{43 a, 43 b}$ C. Helsens,${ }^{35}$

R. C. W. Henderson, ${ }^{87}$ Y. Heng, ${ }^{178}$ S. Henkelmann, ${ }^{172}$ A. M. Henriques Correia,${ }^{35}$ G. H. Herbert, ${ }^{19}$ H. Herde,${ }^{26}$ V. Herget, ${ }^{174}$ Y. Hernández Jiménez, ${ }^{32 c}$ H. Herr, ${ }^{97}$ M. G. Herrmann, ${ }^{112}$ T. Herrmann, ${ }^{46}$ G. Herten, ${ }^{50}$ R. Hertenberger, ${ }^{112}$ L. Hervas, ${ }^{35}$

T. C. Herwig, ${ }^{134}$ G. G. Hesketh, ${ }^{92}$ N. P. Hessey, ${ }^{165 a}$ A. Higashida, ${ }^{160}$ S. Higashino ${ }^{79}$ E. Higón-Rodriguez, ${ }^{171}$

K. Hildebrand, ${ }^{36}$ E. Hill, ${ }^{173}$ J. C. Hill, ${ }^{31}$ K. K. Hill, ${ }^{29}$ K. H. Hiller, ${ }^{44}$ S. J. Hillier ${ }^{21}$ M. Hils, ${ }^{46}$ I. Hinchliffe, ${ }^{18}$

F. Hinterkeuser, ${ }^{24}$ M. Hirose, ${ }^{130}$ D. Hirschbuehl, ${ }^{179}$ B. Hiti, ${ }^{89}$ O. Hladik, ${ }^{138}$ D. R. Hlaluku, ${ }^{32 \mathrm{c}}$ X. Hoad, ${ }^{48}$ J. Hobbs, ${ }^{152}$ N. Hod, ${ }^{165 a}$ M. C. Hodgkinson, ${ }^{146}$ A. Hoecker, ${ }^{35}$ M. R. Hoeferkamp, ${ }^{116}$ F. Hoenig, ${ }^{112}$ D. Hohn, ${ }^{50}$ D. Hohov, ${ }^{129}$

T. R. Holmes, ${ }^{36}$ M. Holzbock, ${ }^{112}$ M. Homann, ${ }^{45}$ B. H. Hommels, ${ }^{31}$ S. Honda ${ }^{166}$ T. Honda ${ }^{79}$ T. M. Hong, ${ }^{136}$ A. Hönle, ${ }^{113}$ B. H. Hooberman, ${ }^{170}$ W. H. Hopkins, ${ }^{128}$ Y. Horii, ${ }^{115}$ P. Horn, ${ }^{46}$ A. J. Horton, ${ }^{149}$ L. A. Horyn, ${ }^{36}$ J-Y. Hostachy, ${ }^{56}$ 
A. Hostiuc, ${ }^{145}$ S. Hou, ${ }^{155}$ A. Hoummada, ${ }^{34 a}$ J. Howarth, ${ }^{98}$ J. Hoya, ${ }^{86}$ M. Hrabovsky, ${ }^{127}$ I. Hristova, ${ }^{19}$ J. Hrivnac, ${ }^{129}$ A. Hrynevich, ${ }^{106}$ T. Hryn'ova, ${ }^{5}$ P. J. Hsu, ${ }^{62}$ S.C. Hsu, ${ }^{145}$ Q. Hu, ${ }^{29}$ S. Hu, ${ }^{58 c}$ Y. Huang, ${ }^{15 a}$ Z. Hubacek, ${ }^{139}$ F. Hubaut, ${ }^{99}$ M. Huebner, ${ }^{24}$ F. Huegging, ${ }^{24}$ T. B. Huffman, ${ }^{132}$ M. Huhtinen, ${ }^{35}$ R. F. H. Hunter, ${ }^{33}$ P. Huo, ${ }^{152}$ A. M. Hupe, ${ }^{33}$

N. Huseynov, ${ }^{77, e}$ J. Huston, ${ }^{104}$ J. Huth, ${ }^{57}$ R. Hyneman, ${ }^{103}$ G. Iacobucci, ${ }^{52}$ G. Iakovidis, ${ }^{29}$ I. Ibragimov, ${ }^{148}$ L. Iconomidou-Fayard, ${ }^{129}$ Z. Idrissi, ${ }^{34 \mathrm{e}} \mathrm{P}$. Iengo, ${ }^{35} \mathrm{R}$. Ignazzi, ${ }^{39} \mathrm{O}$. Igonkina, ${ }^{18, \mathrm{dd}} \mathrm{R}$. Iguchi, ${ }^{160} \mathrm{~T}$. Iizawa, ${ }^{52} \mathrm{Y}$. Ikegami, ${ }^{79}$ M. Ikeno, ${ }^{79}$ D. Iliadis, ${ }^{159} \mathrm{~N}$. Ilic, ${ }^{117} \mathrm{~F}$. Iltzsche, ${ }^{46} \mathrm{G}$. Introzzi, ${ }^{68 \mathrm{a}, 68 \mathrm{~b}} \mathrm{M}$. Iodice, ${ }^{72 \mathrm{a}} \mathrm{K}$. Iordanidou, ${ }^{38} \mathrm{~V}$. Ippolito, ${ }^{70 \mathrm{a}, 70 \mathrm{~b}}$

M. F. Isacson, ${ }^{169} \mathrm{~N}$. Ishijima, ${ }^{130} \mathrm{M}$. Ishino, ${ }^{160} \mathrm{M}$. Ishitsuka, ${ }^{162} \mathrm{~W}$. Islam, ${ }^{126} \mathrm{C}$. Issever, ${ }^{132} \mathrm{~S}$. Istin, ${ }^{157} \mathrm{~F}$. Ito, ${ }^{166}$ J. M. Iturbe Ponce, ${ }^{61 a}$ R. Iuppa,${ }^{73 a, 73 b}$ A. Ivina, ${ }^{177}$ H. Iwasaki, ${ }^{79}$ J. M. Izen, ${ }^{42}$ V. Izzo, ${ }^{67 a}$ P. Jacka, ${ }^{138}$ P. Jackson, ${ }^{1}$ R. M. Jacobs, ${ }^{24}$ V. Jain, ${ }^{2}$ G. Jäkel, ${ }^{179}$ K. B. Jakobi, ${ }^{97}$ K. Jakobs,${ }^{50}$ S. Jakobsen, ${ }^{74}$ T. Jakoubek, ${ }^{138}$ D. O. Jamin, ${ }^{126}$ R. Jansky,${ }^{52}$ J. Janssen, ${ }^{24}$ M. Janus, ${ }^{51}$ P. A. Janus, ${ }^{81 a}$ G. Jarlskog,${ }^{94}$ N. Javadov, ${ }^{77, e}$ T. Javůrek, ${ }^{35}$ M. Javurkova, ${ }^{50}$ F. Jeanneau, ${ }^{142}$ L. Jeanty, ${ }^{18}$ J. Jejelava, ${ }^{156 a, e e}$ A. Jelinskas, ${ }^{175}$ P. Jenni,${ }^{50, f f}$ J. Jeong, ${ }^{44}$ N. Jeong, ${ }^{44}$ S. Jézéquel, ${ }^{5} \mathrm{H}$. Ji, ${ }^{178}$ J. Jia ${ }^{152}$ H. Jiang,${ }^{76}$ Y. Jiang, ${ }^{58 \mathrm{a}}$ Z. Jiang, ${ }^{150, \mathrm{gg}}$ S. Jiggins, ${ }^{50}$ F. A. Jimenez Morales, ${ }^{37}$ J. Jimenez Pena, ${ }^{171}$ S. Jin, ${ }^{15 \mathrm{c}}$ A. Jinaru, ${ }^{27 \mathrm{~b}}$ O. Jinnouchi, ${ }^{162}$ H. Jivan, ${ }^{32 c}$ P. Johansson, ${ }^{146}$ K. A. Johns, ${ }^{7}$ C. A. Johnson, ${ }^{63}$ K. Jon-And, ${ }^{43 a, 43 b}$ R. W. L. Jones, ${ }^{87}$ S. D. Jones, ${ }^{153}$ S. Jones, ${ }^{7}$ T. J. Jones, ${ }^{88}$ J. Jongmanns, ${ }^{59 a}$ P. M. Jorge, ${ }^{137 a, 137 b}$ J. Jovicevic, ${ }^{165 a}$ X. Ju, ${ }^{18}$ J. J. Junggeburth, ${ }^{113}$ A. Juste Rozas, ${ }^{14, y}$ A. Kaczmarska, ${ }^{82}$ M. Kado, ${ }^{129}$ H. Kagan, ${ }^{123}$ M. Kagan, ${ }^{150}$ T. Kaji, ${ }^{176}$ E. Kajomovitz, ${ }^{157}$ C. W. Kalderon, ${ }^{94}$ A. Kaluza,${ }^{97}$ S. Kama, ${ }^{41}$ A. Kamenshchikov, ${ }^{121}$ L. Kanjir, ${ }^{89}$ Y. Kano, ${ }^{160}$ V. A. Kantserov ${ }^{110}$ J. Kanzaki, ${ }^{79}$ L. S. Kaplan, ${ }^{178}$ D. Kar, ${ }^{32 c}$ M. J. Kareem, ${ }^{165 b}$ E. Karentzos, ${ }^{10}$ S. N. Karpov, ${ }^{77}$ Z. M. Karpova, ${ }^{77}$ V. Kartvelishvili, ${ }^{87}$ A. N. Karyukhin, ${ }^{121}$ L. Kashif, ${ }^{178}$ R. D. Kass, ${ }^{123}$ A. Kastanas, ${ }^{43 a, 43 b}$ Y. Kataoka, ${ }^{160}$ C. Kato,${ }^{58 d, 58 c}$ J. Katzy ${ }^{44}$ K. Kawade ${ }^{80}$ K. Kawagoe, ${ }^{85}$ T. Kawamoto, ${ }^{160}$ G. Kawamura, ${ }^{51}$ E. F. Kay, ${ }^{88}$ V. F. Kazanin, ${ }^{120 b, 120 a}$ R. Keeler, ${ }^{173}$ R. Kehoe, ${ }^{41}$ J. S. Keller, ${ }^{33}$ E. Kellermann, ${ }^{94}$ J. J. Kempster,${ }^{21}$ J. Kendrick, ${ }^{21}$ O. Kepka, ${ }^{138}$ S. Kersten, ${ }^{179}$ B. P. Kerševan, ${ }^{89}$ S. Ketabchi Haghighat, ${ }^{164}$ R. A. Keyes, ${ }^{101}$ M. Khader, ${ }^{170}$ F. Khalil-Zada, ${ }^{13}$ A. Khanov, ${ }^{126}$ A. G. Kharlamov, ${ }^{120 b, 120 a}$ T. Kharlamova, ${ }^{120 b, 120 a}$ E. E. Khoda, ${ }^{172}$ A. Khodinov, ${ }^{163}$ T. J. Khoo, ${ }^{52}$ E. Khramov, ${ }^{77}$ J. Khubua, ${ }^{156 b}$ S. Kido, ${ }^{80}$ M. Kiehn,${ }^{52}$ C. R. Kilby, ${ }^{91}$ Y. K. Kim, ${ }^{36}$ N. Kimura,${ }^{64 a, 64 c}$ O. M. Kind,${ }^{19}$ B. T. King, ${ }^{88}$ D. Kirchmeier, ${ }^{46}$ J. Kirk, ${ }^{141}$ A. E. Kiryunin, ${ }^{113}$ T. Kishimoto, ${ }^{160}$ D. Kisielewska, ${ }^{81 a}$ V. Kitali, ${ }^{44}$ O. Kivernyk, ${ }^{5}$ E. Kladiva, ${ }^{28 b, a}$ T. Klapdor-Kleingrothaus, ${ }^{50}$ M. H. Klein, ${ }^{103}$ M. Klein, ${ }^{88}$ U. Klein, ${ }^{88}$ K. Kleinknecht, ${ }^{97}$ P. Klimek, ${ }^{119}$ A. Klimentov, ${ }^{29}$ T. Klingl, ${ }^{24}$ T. Klioutchnikova, ${ }^{35}$ F. F. Klitzner, ${ }^{12}$ P. Kluit, ${ }^{118}$ S. Kluth, ${ }^{113}$ E. Kneringer, ${ }^{74}$ E. B. F. G. Knoops, ${ }^{99}$ A. Knue,${ }^{50}$ A. Kobayashi, ${ }^{160}$ D. Kobayashi, ${ }^{85}$ T. Kobayashi, ${ }^{160}$ M. Kobel, ${ }^{46}$ M. Kocian, ${ }^{150}$ P. Kodys, ${ }^{140}$ P. T. Koenig, ${ }^{24}$ T. Koffas, ${ }^{33}$ E. Koffeman, ${ }^{118}$ N. M. Köhler, ${ }^{113}$ T. Koi, ${ }^{150}$ M. Kolb, ${ }^{59 b}$ I. Koletsou, ${ }^{5}$ T. Kondo, ${ }^{79}$ N. Kondrashova ${ }^{58 c}$ K. Köneke, ${ }^{50}$ A. C. König, ${ }^{117}$ T. Kono, ${ }^{79}$ R. Konoplich, ${ }^{122, h h}$ V. Konstantinides, ${ }^{92}$ N. Konstantinidis, ${ }^{92}$ B. Konya ${ }^{94}$ R. Kopeliansky, ${ }^{63}$ S. Koperny, ${ }^{81 a}$ K. Korcyl,${ }^{82}$ K. Kordas, ${ }^{159}$ G. Koren, ${ }^{158}$ A. Korn,${ }^{92}$ I. Korolkov, ${ }^{14}$ E. V. Korolkova, ${ }^{146}$ N. Korotkova, ${ }^{111}$ O. Kortner, ${ }^{113}$ S. Kortner, ${ }^{113}$ T. Kosek, ${ }^{140}$ V. V. Kostyukhin, ${ }^{24}$ A. Kotwal, ${ }^{47}$

A. Koulouris, ${ }^{10}$ A. Kourkoumeli-Charalampidi, ${ }^{68,68 b}$ C. Kourkoumelis, ${ }^{9}$ E. Kourlitis, ${ }^{146}$ V. Kouskoura, ${ }^{29}$

A. B. Kowalewska, ${ }^{82}$ R. Kowalewski, ${ }^{173}$ T. Z. Kowalski, ${ }^{81 a}$ C. Kozakai, ${ }^{160}$ W. Kozanecki, ${ }^{142}$ A. S. Kozhin, ${ }^{121}$ V. A. Kramarenko, ${ }^{111}$ G. Kramberger, ${ }^{89}$ D. Krasnopevtsev, ${ }^{58 a}$ M. W. Krasny, ${ }^{133}$ A. Krasznahorkay, ${ }^{35}$ D. Krauss, ${ }^{113}$ J. A. Kremer, ${ }^{81 a}$ J. Kretzschmar, ${ }^{88}$ P. Krieger, ${ }^{164}$ K. Krizka, ${ }^{18}$ K. Kroeninger, ${ }^{45}$ H. Kroha, ${ }^{113}$ J. Kroll, ${ }^{138}$ J. Kroll, ${ }^{134}$ J. Krstic, ${ }^{16}$ U. Kruchonak, ${ }^{77}$ H. Krüger, ${ }^{24}$ N. Krumnack, ${ }^{76}$ M. C. Kruse, ${ }^{47}$ T. Kubota, ${ }^{102}$ S. Kuday, ${ }^{4 b}$ J. T. Kuechler, ${ }^{179}$ S. Kuehn, ${ }^{35}$ A. Kugel, ${ }^{59 \mathrm{a}}$ T. Kuhl, ${ }^{44}$ V. Kukhtin, ${ }^{77}$ R. Kukla, ${ }^{99}$ Y. Kulchitsky, ${ }^{105, \text { ii }}$ S. Kuleshov, ${ }^{144 \mathrm{~b}}$ Y. P. Kulinich, ${ }^{170}$ M. Kuna,${ }^{56}$ T. Kunigo, ${ }^{83}$ A. Kupco, ${ }^{138}$ T. Kupfer, ${ }^{45}$ O. Kuprash, ${ }^{158}$ H. Kurashige, ${ }^{80}$ L. L. Kurchaninov, ${ }^{165 a}$ Y. A. Kurochkin, ${ }^{105}$ A. Kurova, ${ }^{10}$ M. G. Kurth, ${ }^{15 d}$ E. S. Kuwertz,${ }^{35}$ M. Kuze,${ }^{162}$ J. Kvita, ${ }^{127}$ T. Kwan, ${ }^{101}$ A. La Rosa, ${ }^{113}$ J. L. La Rosa Navarro, ${ }^{78 d}$ L. La Rotonda, ${ }^{40 b, 40 a}$ F. La Ruffa, ${ }^{40 b, 40 a}$ C. Lacasta, ${ }^{171}$ F. Lacava, ${ }^{70 a, 70 b}$ J. Lacey, ${ }^{44}$ D. P. J. Lack, ${ }^{98}$ H. Lacker, ${ }^{19}$ D. Lacour, ${ }^{133}$ E. Ladygin,${ }^{77}$ R. Lafaye, ${ }^{5}$ B. Laforge, ${ }^{133}$ T. Lagouri, ${ }^{32 c}$ S. Lai, ${ }^{51}$ S. Lammers, ${ }^{63}$ W. Lampl, ${ }^{7}$ E. Lançon, ${ }^{29}$ U. Landgraf, ${ }^{50}$ M. P. J. Landon, ${ }^{90}$ M. C. Lanfermann, ${ }^{52}$ V. S. Lang, ${ }^{44}$ J. C. Lange,${ }^{51}$ R. J. Langenberg, ${ }^{35}$ A. J. Lankford, ${ }^{168}$ F. Lanni, ${ }^{29}$ K. Lantzsch, ${ }^{24}$ A. Lanza, ${ }^{68 a}$ A. Lapertosa,${ }^{53 b, 53 a}$ S. Laplace, ${ }^{133}$ J. F. Laporte, ${ }^{142}$ T. Lari, ${ }^{66 a}$ F. Lasagni Manghi, ${ }^{23 b, 23 a}$ M. Lassnig, ${ }^{35}$ T. S. Lau, ${ }^{61 \mathrm{a}}$ A. Laudrain, ${ }^{129}$ M. Lavorgna,${ }^{67,67 b}$ M. Lazzaroni, ${ }^{66 a, 66 b}$ B. Le, ${ }^{102}$ O. Le Dortz, ${ }^{133}$ E. Le Guirriec ${ }^{99}$ E. P. Le Quilleuc, ${ }^{142}$ M. LeBlanc, ${ }^{7}$ T. LeCompte, ${ }^{6}$ F. Ledroit-Guillon, ${ }^{56}$ C. A. Lee, ${ }^{29}$ G. R. Lee, ${ }^{144 a}$ L. Lee,${ }^{57}$ S. C. Lee ${ }^{155}$ B. Lefebvre, ${ }^{101}$ M. Lefebvre, ${ }^{173}$ F. Legger, ${ }^{112}$ C. Leggett, ${ }^{18}$ K. Lehmann, ${ }^{149}$ N. Lehmann, ${ }^{179}$ G. Lehmann Miotto, ${ }^{35}$ W. A. Leight, ${ }^{44}$ A. Leisos, ${ }^{159, j j}$ M. A. L. Leite, ${ }^{78 d}$ R. Leitner, ${ }^{140}$ D. Lellouch, ${ }^{177}$ K. J. C. Leney, ${ }^{92}$ T. Lenz, ${ }^{24}$ B. Lenzi,${ }^{35}$ R. Leone, ${ }^{7}$ S. Leone,${ }^{69 a}$ C. Leonidopoulos, ${ }^{48}$ G. Lerner, ${ }^{153}$ C. Leroy, ${ }^{107}$ R. Les, ${ }^{164}$ A. A. J. Lesage, ${ }^{142}$ C. G. Lester, ${ }^{31}$ M. Levchenko, ${ }^{135}$ J. Levêque, ${ }^{5}$ D. Levin, ${ }^{103}$ L. J. Levinson, ${ }^{177}$ D. Lewis, ${ }^{90}$ B. Li, ${ }^{15 b}$

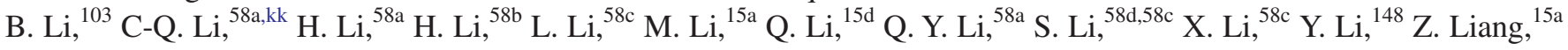


B. Liberti, ${ }^{71 a}$ A. Liblong, ${ }^{164}$ K. Lie, ${ }^{61 \mathrm{c}}$ S. Liem, ${ }^{118}$ A. Limosani, ${ }^{154}$ C. Y. Lin,${ }^{31}$ K. Lin, ${ }^{104}$ T. H. Lin, ${ }^{97}$ R. A. Linck, ${ }^{63}$ J. H. Lindon, ${ }^{21}$ B. E. Lindquist, ${ }^{152}$ A. L. Lionti,${ }^{52}$ E. Lipeles, ${ }^{134}$ A. Lipniacka, ${ }^{17}$ M. Lisovyi, ${ }^{59 b}$ T. M. Liss, ${ }^{170,11}$ A. Lister, ${ }^{172}$ A. M. Litke, ${ }^{143}$ J. D. Little, ${ }^{8}$ B. Liu, ${ }^{76}$ B. L Liu, ${ }^{6}$ H. B. Liu ${ }^{29}$ H. Liu, ${ }^{103}$ J. B. Liu, ${ }^{58 a}$ J. K. K. Liu, ${ }^{132}$ K. Liu, ${ }^{133}$ M. Liu, ${ }^{58 a}$ P. Liu, ${ }^{18}$ Y. Liu, ${ }^{15 a}$ Y. L. Liu, ${ }^{58 a}$ Y. W. Liu, ${ }^{58 a}$ M. Livan, ${ }^{68 a, 68 b}$ A. Lleres, ${ }^{56}$ J. Llorente Merino, ${ }^{15 a}$ S. L. Lloyd ${ }^{90}$ C. Y. Lo, ${ }^{61 b}$ F. Lo Sterzo ${ }^{41}$ E. M. Lobodzinska, ${ }^{44}$ P. Loch, ${ }^{7}$ T. Lohse, ${ }^{19}$ K. Lohwasser ${ }^{146}$ M. Lokajicek, ${ }^{138}$ J. D. Long, ${ }^{170}$ R. E. Long ${ }^{87}$ L. Longo, ${ }^{65 a, 65 b}$ K. A. Looper, ${ }^{123}$ J. A. Lopez, ${ }^{144 b}$ I. Lopez Paz, ${ }^{98}$ A. Lopez Solis, ${ }^{146}$ J. Lorenz, ${ }^{112}$ N. Lorenzo Martinez, ${ }^{5}$ M. Losada, ${ }^{22}$ P. J. Lösel, ${ }^{112}$ A. Lösle ${ }^{50}$ X. Lou, ${ }^{44}$ X. Lou, ${ }^{15 a}$ A. Lounis, ${ }^{129}$ J. Love, ${ }^{6}$ P. A. Love, ${ }^{87}$ J. J. Lozano Bahilo, ${ }^{171}$ H. Lu, ${ }^{61 a}$ M. Lu, ${ }^{58 a}$ Y. J. Lu, ${ }^{62}$ H. J. Lubatti, ${ }^{145}$ C. Luci, ${ }^{70 a, 70 b}$ A. Lucotte,${ }^{56}$ C. Luedtke,${ }^{50}$ F. Luehring, ${ }^{63}$ I. Luise, ${ }^{133}$ L. Luminari, ${ }^{70 a}$ B. Lund-Jensen, ${ }^{151}$ M. S. Lutz, ${ }^{100}$ P. M. Luzi ${ }^{133}$ D. Lynn, ${ }^{29}$ R. Lysak, ${ }^{138}$ E. Lytken, ${ }^{94}$ F. Lyu, ${ }^{15 a}$ V. Lyubushkin, ${ }^{77}$ T. Lyubushkina, ${ }^{77}$ H. Ma ${ }^{29}$ L. L. Ma ${ }^{58 \mathrm{~b}}$ Y. Ma ${ }^{58 \mathrm{~b}}$ G. Maccarrone, ${ }^{49}$ A. Macchiolo, ${ }^{113}$ C. M. Macdonald, ${ }^{146}$ J. Machado Miguens, ${ }^{134,137 b}$ D. Madaffari, ${ }^{171}$ R. Madar, ${ }^{37}$ W. F. Mader, ${ }^{46}$ A. Madsen,${ }^{44}$ N. Madysa, ${ }^{46}$ J. Maeda, ${ }^{80}$ K. Maekawa, ${ }^{160}$ S. Maeland,${ }^{17}$ T. Maeno, ${ }^{29}$ M. Maerker ${ }^{46}$ A. S. Maevskiy, ${ }^{111}$ V. Magerl, ${ }^{50}$ D. J. Mahon, ${ }^{38}$ C. Maidantchik ${ }^{78 b}$ T. Maier, ${ }^{112}$ A. Maio, ${ }^{137 a, 137 b, 137 \mathrm{~d}}$ O. Majersky, ${ }^{28 \mathrm{a}}$ S. Majewski, ${ }^{128}$ Y. Makida, ${ }^{79}$ N. Makovec, ${ }^{129}$ B. Malaescu, ${ }^{133}$ Pa. Malecki, ${ }^{82}$ V. P. Maleev, ${ }^{135}$ F. Malek, ${ }^{56}$ U. Mallik, ${ }^{75}$ D. Malon, ${ }^{6}$ C. Malone, ${ }^{31}$ S. Maltezos, ${ }^{10}$ S. Malyukov ${ }^{35}$ J. Mamuzic, ${ }^{171}$ G. Mancini, ${ }^{49}$ I. Mandić, ${ }^{89}$ J. Maneira, ${ }^{137 a}$ L. Manhaes de Andrade Filho, ${ }^{78 a}$ J. Manjarres Ramos, ${ }^{46}$ K. H. Mankinen, ${ }^{94}$ A. Mann, ${ }^{112}$ A. Manousos, ${ }^{74}$ B. Mansoulie, ${ }^{142}$ J. D. Mansour, ${ }^{15 a}$ S. Manzoni, ${ }^{66 a, 66 b}$ A. Marantis, ${ }^{159}$ G. Marceca, ${ }^{30}$ L. March, ${ }^{52}$ L. Marchese, ${ }^{132}$ G. Marchiori, ${ }^{133}$ M. Marcisovsky, ${ }^{138}$ C. Marcon, ${ }^{94}$ C. A. Marin Tobon, ${ }^{35}$ M. Marjanovic, ${ }^{37}$ F. Marroquim, ${ }^{78 b}$ Z. Marshall,${ }^{18}$ M. U. F Martensson, ${ }^{169}$ S. Marti-Garcia, ${ }^{171}$ C. B. Martin, ${ }^{123}$ T. A. Martin, ${ }^{175}$ V. J. Martin, ${ }^{48}$ B. Martin dit Latour, ${ }^{17}$ M. Martinez, ${ }^{14, y}$ V. I. Martinez Outschoorn, ${ }^{100}$ S. Martin-Haugh, ${ }^{141}$ V. S. Martoiu, ${ }^{27 b}$ A. C. Martyniuk, ${ }^{92}$ A. Marzin, ${ }^{35}$ L. Masetti, ${ }^{97}$ T. Mashimo, ${ }^{160}$ R. Mashinistov, ${ }^{108}$ J. Masik, ${ }^{98}$ A. L. Maslennikov, ${ }^{120 b, 120 a}$ L. H. Mason, ${ }^{102}$ L. Massa, ${ }^{71,71 b}$ P. Massarotti, ${ }^{67,67 b}$ P. Mastrandrea, ${ }^{152}$

A. Mastroberardino, ${ }^{40 \mathrm{~b}, 40 \mathrm{a}}$ T. Masubuchi, ${ }^{160}$ P. Mättig, ${ }^{179}$ J. Maurer, ${ }^{27 \mathrm{~b}}$ B. Maček ${ }^{89}$ S. J. Maxfield ${ }^{88}$ D. A. Maximov, ${ }^{120 b, 120 \mathrm{a}}$ R. Mazini, ${ }^{155}$ I. Maznas, ${ }^{159}$ S. M. Mazza, ${ }^{143}$ G. Mc Goldrick, ${ }^{164}$ S. P. Mc Kee, ${ }^{103}$ A. McCarn, ${ }^{41}$ T. G. McCarthy, ${ }^{113}$ L. I. McClymont, ${ }^{92}$ W. P. McCormack, ${ }^{18}$ E. F. McDonald, ${ }^{102}$ J. A. Mcfayden, ${ }^{35}$ G. Mchedlidze,${ }^{51}$ M. A. McKay, ${ }^{41}$ K. D. McLean, ${ }^{173}$ S. J. McMahon, ${ }^{141}$ P. C. McNamara, ${ }^{102}$ C. J. McNicol, ${ }^{175}$ R. A. McPherson, ${ }^{173,0}$ J. E. Mdhluli, ${ }^{32 c}$ Z. A. Meadows, ${ }^{100}$ S. Meehan, ${ }^{145}$ T. M. Megy, ${ }^{50}$ S. Mehlhase, ${ }^{112}$ A. Mehta ${ }^{88}$ T. Meideck,${ }^{56}$ B. Meirose ${ }^{42}$ D. Melini, ${ }^{171, m m}$ B. R. Mellado Garcia, ${ }^{32 c}$ J. D. Mellenthin,${ }^{51}$ M. Melo, ${ }^{28 a}$ F. Meloni,${ }^{44}$ A. Melzer, ${ }^{24}$ S. B. Menary, ${ }^{98}$

E. D. Mendes Gouveia, ${ }^{137 a}$ L. Meng, ${ }^{88}$ X. T. Meng, ${ }^{103}$ S. Menke, ${ }^{113}$ E. Meoni, ${ }^{40 b, 40 a}$ S. Mergelmeyer, ${ }^{19}$ S. A. M. Merkt, ${ }^{136}$ C. Merlassino, ${ }^{20}$ P. Mermod, ${ }^{52}$ L. Merola,${ }^{67,67 b}$ C. Meroni, ${ }^{66 a}$ F. S. Merritt, ${ }^{36}$ A. Messina, ${ }^{70 a, 70 b}$ J. Metcalfe, ${ }^{6}$ A. S. Mete, ${ }^{168}$ C. Meyer, ${ }^{134}$ J. Meyer, ${ }^{157}$ J-P. Meyer, ${ }^{142}$ H. Meyer Zu Theenhausen, ${ }^{59 a}$ F. Miano, ${ }^{153}$ R. P. Middleton, ${ }^{141}$ L. Mijović, ${ }^{48}$ G. Mikenberg, ${ }^{177}$ M. Mikestikova, ${ }^{138}$ M. Mikuž, ${ }^{89}$ M. Milesi, ${ }^{102}$ A. Milic, ${ }^{164}$ D. A. Millar, ${ }^{90}$ D. W. Miller, ${ }^{36}$ A. Milov, ${ }^{177}$ D. A. Milstead ${ }^{43 a, 43 b}$ R. A. Mina, ${ }^{150, g g}$ A. A. Minaenko, ${ }^{121}$ M. Miñano Moya, ${ }^{171}$ I. A. Minashvili, ${ }^{156 b}$ A. I. Mincer, ${ }^{122}$ B. Mindur, ${ }^{81 \mathrm{a}}$ M. Mineev, ${ }^{77}$ Y. Minegishi, ${ }^{160}$ Y. Ming, ${ }^{178}$ L. M. Mir, ${ }^{14}$ A. Mirto, ${ }^{65 a, 65 b}$ K. P. Mistry, ${ }^{134}$ T. Mitani, ${ }^{176}$ J. Mitrevski, ${ }^{112}$ V. A. Mitsou, ${ }^{171}$ M. Mittal,${ }^{58 c}$ A. Miucci, ${ }^{20}$ P. S. Miyagawa, ${ }^{146}$ A. Mizukami, ${ }^{79}$ J. U. Mjörnmark, ${ }^{94}$ T. Mkrtchyan, ${ }^{181}$ M. Mlynarikova, ${ }^{140}$ T. Moa, ${ }^{43 a, 43 b}$ K. Mochizuki, ${ }^{107}$ P. Mogg, ${ }^{50}$ S. Mohapatra, ${ }^{38}$ S. Molander, ${ }^{43 a, 43 b}$ R. Moles-Valls, ${ }^{24}$ M. C. Mondragon, ${ }^{104}$ K. Mönig, ${ }^{44}$ J. Monk ${ }^{39}$ E. Monnier, ${ }^{99}$ A. Montalbano, ${ }^{149}$ J. Montejo Berlingen, ${ }^{35}$ F. Monticelli, ${ }^{86}$ S. Monzani, ${ }^{66 \mathrm{a}}$ N. Morange, ${ }^{129}$ D. Moreno, ${ }^{22}$ M. Moreno Llácer, ${ }^{35}$ P. Morettini, ${ }^{53 \mathrm{~b}}$ M. Morgenstern, ${ }^{118}$ S. Morgenstern, ${ }^{46}$ D. Mori ${ }^{149}$ M. Morii, ${ }^{57}$ M. Morinaga, ${ }^{176}$ V. Morisbak, ${ }^{131}$ A. K. Morley, ${ }^{35}$ G. Mornacchi, ${ }^{35}$ A. P. Morris,${ }^{92}$ J. D. Morris, ${ }^{90}$ L. Morvaj, ${ }^{152}$ P. Moschovakos,${ }^{10}$ M. Mosidze, ${ }^{156 \mathrm{~b}}$ H. J. Moss, ${ }^{146}$ J. Moss, ${ }^{150, \mathrm{nn}}$ K. Motohashi, ${ }^{162}$ R. Mount, ${ }^{150}$ E. Mountricha, ${ }^{35}$ E. J. W. Moyse, ${ }^{100}$ S. Muanza, ${ }^{99}$ F. Mueller, ${ }^{113}$ J. Mueller, ${ }^{136}$ R. S. P. Mueller, ${ }^{112}$ D. Muenstermann, ${ }^{87}$ G. A. Mullier ${ }^{94}$ F. J. Munoz Sanchez,${ }^{98}$ P. Murin,${ }^{28 b}$ W. J. Murray,${ }^{175,141}$ A. Murrone, ${ }^{66 a, 66 b}$ M. Muškinja,${ }^{89}$ C. Mwewa, ${ }^{32 a}$ A. G. Myagkov, ${ }^{121, o 0}$ J. Myers, ${ }^{128}$ M. Myska, ${ }^{139}$ B. P. Nachman, ${ }^{18}$ O. Nackenhorst,${ }^{45}$ K. Nagai, ${ }^{132}$ K. Nagano, ${ }^{79}$ Y. Nagasaka, ${ }^{60}$ M. Nagel, ${ }^{50}$ E. Nagy, ${ }^{99}$ A. M. Nairz,${ }^{35}$ Y. Nakahama, ${ }^{115}$ K. Nakamura, ${ }^{79}$ T. Nakamura ${ }^{160}$ I. Nakano, ${ }^{124}$ H. Nanjo, ${ }^{130}$ F. Napolitano, ${ }^{59 a}$ R. F. Naranjo Garcia, ${ }^{44}$ R. Narayan, ${ }^{11}$ D. I. Narrias Villar, ${ }^{59 a}$ I. Naryshkin ${ }^{135}$ T. Naumann, ${ }^{44}$ G. Navarro, ${ }^{22}$ R. Nayyar, ${ }^{7}$ H. A. Neal, ${ }^{103, \mathrm{a}}$ P. Y. Nechaeva, ${ }^{108}$ T. J. Neep, ${ }^{142}$ A. Negri, ${ }^{68 a, 68 b}$ M. Negrini, ${ }^{23 b}$ S. Nektarijevic, ${ }^{117}$ C. Nellist, ${ }^{51}$ M. E. Nelson, ${ }^{132}$ S. Nemecek, ${ }^{138}$ P. Nemethy, ${ }^{122}$ M. Nessi,,${ }^{35, p p}$ M. S. Neubauer, ${ }^{170}$ M. Neumann, ${ }^{179}$ P. R. Newman, ${ }^{21}$ T. Y. Ng, ${ }^{61 \mathrm{c}}$ Y. S. Ng, ${ }^{19}$ Y. W. Y. Ng, ${ }^{168}$ H. D. N. Nguyen, ${ }^{99}$ T. Nguyen Manh,${ }^{107}$ E. Nibigira, ${ }^{37}$ R. B. Nickerson, ${ }^{132}$ R. Nicolaidou, ${ }^{142}$ D. S. Nielsen, ${ }^{39}$ J. Nielsen, ${ }^{143}$ N. Nikiforou, ${ }^{11}$ V. Nikolaenko, ${ }^{121,00}$ I. Nikolic-Audit, ${ }^{133}$ K. Nikolopoulos, ${ }^{21}$ P. Nilsson, ${ }^{29}$ Y. Ninomiya, ${ }^{79}$ A. Nisati, ${ }^{70 a}$ N. Nishu, ${ }^{58 c}$ R. Nisius, ${ }^{113}$ I. Nitsche, ${ }^{45}$ T. Nitta, ${ }^{176}$ T. Nobe, ${ }^{160}$ 
Y. Noguchi, ${ }^{83}$ M. Nomachi, ${ }^{130}$ I. Nomidis, ${ }^{133}$ M. A. Nomura, ${ }^{29}$ T. Nooney,${ }^{90}$ M. Nordberg, ${ }^{35}$ N. Norjoharuddeen, ${ }^{132}$ T. Novak, ${ }^{89}$ O. Novgorodova, ${ }^{46}$ R. Novotny, ${ }^{139}$ L. Nozka, ${ }^{127}$ K. Ntekas, ${ }^{168}$ E. Nurse, ${ }^{92}$ F. Nuti, ${ }^{102}$ F. G. Oakham, ${ }^{33, f}$ H. Oberlack, ${ }^{113}$ J. Ocariz, ${ }^{133}$ A. Ochi, ${ }^{80}$ I. Ochoa, ${ }^{38}$ J. P. Ochoa-Ricoux, ${ }^{144 a}$ K. O'Connor, ${ }^{26}$ S. Oda ${ }^{85}$ S. Odaka, ${ }^{79}$ S. Oerdek,${ }^{51}$ A. Oh, ${ }^{98}$ S. H. Oh, ${ }^{47}$ C. C. Ohm, ${ }^{151}$ H. Oide, ${ }^{53 b, 53 a}$ M. L. Ojeda,${ }^{164}$ H. Okawa ${ }^{166}$ Y. Okazaki, ${ }^{83}$ Y. Okumura, ${ }^{160}$ T. Okuyama, ${ }^{79}$ A. Olariu, ${ }^{27 b}$ L. F. Oleiro Seabra, ${ }^{137 a}$ S. A. Olivares Pino, ${ }^{144 a}$ D. Oliveira Damazio, ${ }^{29}$ J. L. Oliver, ${ }^{1}$ M. J. R. Olsson, ${ }^{36}$ A. Olszewski, ${ }^{82}$ J. Olszowska, ${ }^{82}$ D. C. O'Neil, ${ }^{149}$ A. Onofre, ${ }^{137 a, 137 e}$ K. Onogi, ${ }^{115}$ P. U. E. Onyisi, ${ }^{11}$ H. Oppen, ${ }^{131}$ M. J. Oreglia, ${ }^{36}$ G. E. Orellana, ${ }^{86}$ Y. Oren, ${ }^{158}$ D. Orestano, ${ }^{72 a, 72 b}$ E. C. Orgill, ${ }^{98}$ N. Orlando, ${ }^{61 b}$ A. A. O'Rourke, ${ }^{44}$ R. S. Orr, ${ }^{164}$ B. Osculati, ${ }^{53 b, 53 a, a}$ V. O'Shea, ${ }^{55}$ R. Ospanov, ${ }^{58 a}$ G. Otero y Garzon, ${ }^{30}$ H. Otono, ${ }^{85}$ M. Ouchrif, ${ }^{34 \mathrm{~d}}$ F. Ould-Saada, ${ }^{131}$ A. Ouraou, ${ }^{142}$ Q. Ouyang, ${ }^{15 a}$ M. Owen, ${ }^{55}$ R. E. Owen, ${ }^{21}$ V. E. Ozcan, ${ }^{12 c}$ N. Ozturk, ${ }^{8}$ J. Pacalt, ${ }^{127}$ H. A. Pacey, ${ }^{31}$ K. Pachal,${ }^{149}$ A. Pacheco Pages,${ }^{14}$ L. Pacheco Rodriguez, ${ }^{142}$ C. Padilla Aranda, ${ }^{14}$ S. Pagan Griso, ${ }^{18}$ M. Paganini, ${ }^{180}$ G. Palacino, ${ }^{63}$ S. Palazzo, ${ }^{48}$ S. Palestini,${ }^{35}$ M. Palka, ${ }^{81 b}$ D. Pallin, ${ }^{37}$ I. Panagoulias, ${ }^{10}$ C. E. Pandini, ${ }^{35}$ J. G. Panduro Vazquez, ${ }^{91}$ P. Pani, ${ }^{35}$ G. Panizzo, ${ }^{64 a, 64 c}$ L. Paolozzi, ${ }^{52}$ T. D. Papadopoulou, ${ }^{10}$ K. Papageorgiou, ${ }^{9, u}$ A. Paramonov, ${ }^{6}$ D. Paredes Hernandez, ${ }^{61 b}$ S. R. Paredes Saenz, ${ }^{132}$ B. Parida, ${ }^{163}$ T. H. Park,${ }^{33}$ A. J. Parker, ${ }^{87}$ K. A. Parker, ${ }^{44}$ M. A. Parker, ${ }^{31}$ F. Parodi, ${ }^{53 b, 53 a}$ J. A. Parsons, ${ }^{38}$ U. Parzefall, ${ }^{50}$ V. R. Pascuzzi, ${ }^{164}$ J. M. P. Pasner, ${ }^{143}$ E. Pasqualucci, ${ }^{70 a}$ S. Passaggio,${ }^{53 b}$ F. Pastore, ${ }^{91}$ P. Pasuwan, ${ }^{43 a, 43 b}$ S. Pataraia, ${ }^{97}$ J. R. Pater, ${ }^{98}$ A. Pathak, ${ }^{178, \mathrm{~g}}$ T. Pauly, ${ }^{35}$ B. Pearson, ${ }^{113}$ M. Pedersen, ${ }^{131}$ L. Pedraza Diaz, ${ }^{117}$ R. Pedro, ${ }^{137 a, 137 \mathrm{~b}}$ S. V. Peleganchuk, ${ }^{120 \mathrm{~b}, 120 \mathrm{a}}$ O. Penc, ${ }^{138}$ C. Peng, ${ }^{15 \mathrm{~d}}$ H. Peng, ${ }^{58 \mathrm{a}}$ B. S. Peralva, ${ }^{78 \mathrm{a}}$ M. M. Perego, ${ }^{129}$ A. P. Pereira Peixoto, ${ }^{137 \mathrm{a}}$ D. V. Perepelitsa, ${ }^{29}$ F. Peri, ${ }^{19}$ L. Perini, ${ }^{66 a, 66 b}$ H. Pernegger, ${ }^{35}$ S. Perrella,${ }^{67 a, 67 b}$ V. D. Peshekhonov, ${ }^{77, a}$ K. Peters,${ }^{44}$ R. F. Y. Peters, ${ }^{98}$ B. A. Petersen,${ }^{35}$ T. C. Petersen, ${ }^{39}$ E. Petit, ${ }^{56}$ A. Petridis, ${ }^{1}$ C. Petridou, ${ }^{159}$ P. Petroff, ${ }^{129}$ M. Petrov, ${ }^{132}$ F. Petrucci, ${ }^{72 a, 72 b}$ M. Pettee, ${ }^{180}$ N. E. Pettersson, ${ }^{100}$ A. Peyaud, ${ }^{142}$ R. Pezoa, ${ }^{144 b}$ T. Pham, ${ }^{102}$ F. H. Phillips,${ }^{104}$ P. W. Phillips, ${ }^{141}$ M. W. Phipps, ${ }^{170}$ G. Piacquadio, ${ }^{152}$ E. Pianori, ${ }^{18}$ A. Picazio, ${ }^{100}$ R. H. Pickles, ${ }^{98}$ R. Piegaia,${ }^{30}$ J. E. Pilcher, ${ }^{36}$ A. D. Pilkington, ${ }^{98}$ M. Pinamonti, ${ }^{71 a, 71 b}$ J. L. Pinfold, ${ }^{3}$ M. Pitt, ${ }^{177}$ L. Pizzimento, ${ }^{71 a, 71 b}$ M.-A. Pleier, ${ }^{29}$ V. Pleskot,${ }^{140}$ E. Plotnikova, ${ }^{77}$ D. Pluth, ${ }^{76}$ P. Podberezko, ${ }^{120 b, 120 \mathrm{a}}$ R. Poettgen, ${ }^{94}$ R. Poggi, ${ }^{52}$ L. Poggioli, ${ }^{129}$ I. Pogrebnyak, ${ }^{104}$ D. Pohl,${ }^{24}$ I. Pokharel,${ }^{51}$ G. Polesello, ${ }^{68 a}$ A. Poley, ${ }^{18}$ A. Policicchio, ${ }^{70 a, 70 b}$ R. Polifka, ${ }^{35}$ A. Polini, ${ }^{23 b}$ C. S. Pollard, ${ }^{44}$ V. Polychronakos, ${ }^{29}$ D. Ponomarenko, ${ }^{110}$ L. Pontecorvo, ${ }^{35}$ G. A. Popeneciu, ${ }^{27 \mathrm{~d}}$ D. M. Portillo Quintero, ${ }^{133}$ S. Pospisil, ${ }^{139}$ K. Potamianos, ${ }^{44}$ I. N. Potrap,${ }^{77}$ C. J. Potter, ${ }^{31}$ H. Potti, ${ }^{11}$ T. Poulsen, ${ }^{94}$ J. Poveda, ${ }^{35}$ T. D. Powell, ${ }^{146}$ M. E. Pozo Astigarraga, ${ }^{35}$ P. Pralavorio, ${ }^{99}$ S. Prell, ${ }^{76}$ D. Price, ${ }^{98}$ M. Primavera, ${ }^{65 \mathrm{a}}$ S. Prince, ${ }^{101}$ M. L. Proffitt, ${ }^{145}$ N. Proklova, ${ }^{110}$ K. Prokofiev, ${ }^{61 \mathrm{c}}$ F. Prokoshin, ${ }^{144 \mathrm{~b}}$

S. Protopopescu, ${ }^{29}$ J. Proudfoot, ${ }^{6}$ M. Przybycien, ${ }^{81 a}$ A. Puri, ${ }^{170}$ P. Puzo, ${ }^{129}$ J. Qian, ${ }^{103}$ Y. Qin, ${ }^{98}$ A. Quadt, ${ }^{51}$ M. Queitsch-Maitland, ${ }^{44}$ A. Qureshi, ${ }^{1}$ P. Rados, ${ }^{102}$ F. Ragusa, ${ }^{66 a, 66 b}$ G. Rahal, ${ }^{95}$ J. A. Raine, ${ }^{52}$ S. Rajagopalan, ${ }^{29}$ A. Ramirez Morales, ${ }^{90}$ K. Ran, ${ }^{15 \mathrm{a}}$ T. Rashid, ${ }^{129}$ S. Raspopov,${ }^{5}$ M. G. Ratti, ${ }^{66 a, 66 \mathrm{~b}}$ D. M. Rauch, ${ }^{44}$ F. Rauscher, ${ }^{112}$ S. Rave, ${ }^{97}$ B. Ravina, ${ }^{146}$ I. Ravinovich, ${ }^{177}$ J. H. Rawling, ${ }^{98}$ M. Raymond, ${ }^{35}$ A. L. Read, ${ }^{131}$ N. P. Readioff, ${ }^{56}$ M. Reale, ${ }^{65 a, 65 b}$ D. M. Rebuzzi, ${ }^{68 a, 68 b}$ A. Redelbach, ${ }^{174}$ G. Redlinger ${ }^{29}$ R. Reece, ${ }^{143}$ R. G. Reed, ${ }^{32 \mathrm{c}}$ K. Reeves,${ }^{42}$ L. Rehnisch,${ }^{19}$

J. Reichert, ${ }^{134}$ D. Reikher, ${ }^{158}$ A. Reiss, ${ }^{97}$ A. Rej, ${ }^{148}$ C. Rembser,${ }^{35}$ H. Ren, ${ }^{15 \mathrm{~d}}$ M. Rescigno, ${ }^{70 a}$ S. Resconi, ${ }^{66 a}$ E. D. Resseguie, ${ }^{134}$ S. Rettie, ${ }^{172}$ E. Reynolds, ${ }^{21}$ O. L. Rezanova, ${ }^{120 b, 120 a}$ P. Reznicek, ${ }^{140}$ E. Ricci, ${ }^{73 a, 73 b}$ R. Richter, ${ }^{113}$ S. Richter, ${ }^{44}$ E. Richter-Was, ${ }^{81 b}$ O. Ricken, ${ }^{24}$ M. Ridel, ${ }^{133}$ P. Rieck, ${ }^{113}$ C. J. Riegel,${ }^{179}$ O. Rifki, ${ }^{44}$ M. Rijssenbeek, ${ }^{152}$ A. Rimoldi, ${ }^{68 a, 68 b}$ M. Rimoldi, ${ }^{20}$ L. Rinaldi, ${ }^{23 b}$ G. Ripellino, ${ }^{151}$ B. Ristić, ${ }^{87}$ E. Ritsch, ${ }^{35}$ I. Riu, ${ }^{14}$ J. C. Rivera Vergara, ${ }^{144 a}$ F. Rizatdinova, ${ }^{126}$ E. Rizvi, ${ }^{90}$ C. Rizzi, ${ }^{14}$ R. T. Roberts, ${ }^{98}$ S. H. Robertson, ${ }^{101, o}$ D. Robinson, ${ }^{31}$ J. E. M. Robinson, ${ }^{44}$ A. Robson, ${ }^{55}$ E. Rocco, ${ }^{97}$ C. Roda,${ }^{69 a, 69 b}$ Y. Rodina,${ }^{99}$ S. Rodriguez Bosca, ${ }^{171}$ A. Rodriguez Perez,${ }^{14}$

D. Rodriguez Rodriguez, ${ }^{171}$ A. M. Rodríguez Vera, ${ }^{165 b}$ S. Roe, ${ }^{35}$ C. S. Rogan, ${ }^{57}$ O. Røhne, ${ }^{131}$ R. Röhrig, ${ }^{113}$ C. P. A. Roland, ${ }^{63}$ J. Roloff, ${ }^{57}$ A. Romaniouk, ${ }^{110}$ M. Romano, ${ }^{23 b, 23 a}$ N. Rompotis, ${ }^{88}$ M. Ronzani,${ }^{122}$ L. Roos,${ }^{133}$ S. Rosati, ${ }^{70 a}$ K. Rosbach, ${ }^{50}$ N-A. Rosien, ${ }^{51}$ B. J. Rosser, ${ }^{134}$ E. Rossi, ${ }^{44}$ E. Rossi, ${ }^{72 a, 72 b}$ E. Rossi, ${ }^{67 a, 67 b}$ L. P. Rossi, ${ }^{53 b}$ L. Rossini, ${ }^{66 a, 66 b}$ J. H. N. Rosten, ${ }^{31}$ R. Rosten, ${ }^{14}$ M. Rotaru, ${ }^{27 b}$ J. Rothberg, ${ }^{145}$ D. Rousseau, ${ }^{129}$ D. Roy, ${ }^{32 c}$ A. Rozanov, ${ }^{99}$ Y. Rozen, ${ }^{157}$ X. Ruan, ${ }^{32 \mathrm{c}}$ F. Rubbo, ${ }^{150}$ F. Rühr, ${ }^{50}$ A. Ruiz-Martinez, ${ }^{171}$ Z. Rurikova, ${ }^{50}$ N. A. Rusakovich,${ }^{77}$ H. L. Russell, ${ }^{101}$ J. P. Rutherfoord, ${ }^{7}$ E. M. Rüttinger, ${ }^{44, q q}$ Y. F. Ryabov, ${ }^{135}$ M. Rybar, ${ }^{38}$ G. Rybkin, ${ }^{129}$ S. Ryu, ${ }^{6}$ A. Ryzhov, ${ }^{121}$ G. F. Rzehorz, ${ }^{51}$ P. Sabatini, ${ }^{51}$ G. Sabato, ${ }^{118}$ S. Sacerdoti, ${ }^{129}$ H. F-W. Sadrozinski, ${ }^{143}$ R. Sadykov, ${ }^{77}$ F. Safai Tehrani, ${ }^{70 a}$ P. Saha, ${ }^{119}$ M. Sahinsoy, ${ }^{59 a}$ A. Sahu, ${ }^{179}$ M. Saimpert, ${ }^{44}$ M. Saito, ${ }^{160}$ T. Saito, ${ }^{160}$ H. Sakamoto, ${ }^{160}$ A. Sakharov, ${ }^{122, \text { hh }}$ D. Salamani, ${ }^{52}$ G. Salamanna, ${ }^{72 a, 72 b}$ J. E. Salazar Loyola, ${ }^{144 b}$ P. H. Sales De Bruin, ${ }^{169}$ D. Salihagic, ${ }^{113}$ A. Salnikov, ${ }^{150}$ J. Salt, ${ }^{171}$ D. Salvatore, ${ }^{40 b, 40 a}$ F. Salvatore, ${ }^{153}$ A. Salvucci, ${ }^{61 a, 61 b, 61 \mathrm{c}}$ A. Salzburger,${ }^{35}$ J. Samarati, ${ }^{35}$ D. Sammel,${ }^{50}$ D. Sampsonidis, ${ }^{159}$ D. Sampsonidou, ${ }^{159}$ J. Sánchez, ${ }^{171}$ A. Sanchez Pineda, ${ }^{64 a, 64 c}$ H. Sandaker, ${ }^{131}$ C. O. Sander, ${ }^{44}$ M. Sandhoff, ${ }^{179}$ C. Sandoval, ${ }^{22}$ 
D. P. C. Sankey, ${ }^{141}$ M. Sannino, ${ }^{53 b, 53 a}$ Y. Sano, ${ }^{115}$ A. Sansoni,${ }^{49}$ C. Santoni,${ }^{37}$ H. Santos, ${ }^{137 a}$ I. Santoyo Castillo, ${ }^{153}$ A. Santra, ${ }^{171}$ A. Sapronov, ${ }^{77}$ J. G. Saraiva, ${ }^{137 a, 137 d}$ O. Sasaki, ${ }^{79}$ K. Sato, ${ }^{166}$ E. Sauvan, ${ }^{5}$ P. Savard, ${ }^{164, \mathrm{f}}$ N. Savic, ${ }^{113}$ R. Sawada, ${ }^{160}$ C. Sawyer, ${ }^{141}$ L. Sawyer, ${ }^{93, w}$ C. Sbarra, ${ }^{23 b}$ A. Sbrizzi, ${ }^{23 a}$ T. Scanlon, ${ }^{92}$ J. Schaarschmidt, ${ }^{145}$ P. Schacht, ${ }^{113}$ B. M. Schachtner, ${ }^{112}$ D. Schaefer, ${ }^{36}$ L. Schaefer, ${ }^{134}$ J. Schaeffer,${ }^{97}$ S. Schaepe,${ }^{35}$ U. Schäfer, ${ }^{97}$ A. C. Schaffer, ${ }^{129}$ D. Schaile, ${ }^{112}$ R. D. Schamberger, ${ }^{152}$ N. Scharmberg, ${ }^{98}$ V. A. Schegelsky, ${ }^{135}$ D. Scheirich, ${ }^{140}$ F. Schenck, ${ }^{19}$ M. Schernau, ${ }^{168}$ C. Schiavi, ${ }^{53 b, 53 a}$ S. Schier, ${ }^{143}$ L. K. Schildgen, ${ }^{24}$ Z. M. Schillaci, ${ }^{26}$ E. J. Schioppa, ${ }^{35}$ M. Schioppa, ${ }^{40 b, 40 a}$ K. E. Schleicher, ${ }^{50}$ S. Schlenker, ${ }^{35}$ K. R. Schmidt-Sommerfeld, ${ }^{113}$ K. Schmieden, ${ }^{35}$ C. Schmitt, ${ }^{97}$ S. Schmitt, ${ }^{44}$ S. Schmitz, ${ }^{97}$ J. C. Schmoeckel, ${ }^{44}$ U. Schnoor, ${ }^{50}$ L. Schoeffel, ${ }^{142}$ A. Schoening, ${ }^{59 b}$ E. Schopf, ${ }^{132}$ M. Schott, ${ }^{97}$ J. F. P. Schouwenberg, ${ }^{117}$ J. Schovancova, ${ }^{35}$ S. Schramm, ${ }^{52}$ A. Schulte, ${ }^{97}$ H-C. Schultz-Coulon, ${ }^{59 a}$ M. Schumacher, ${ }^{50}$ B. A. Schumm, ${ }^{143}$ Ph. Schune, ${ }^{142}$ A. Schwartzman, ${ }^{150}$ T. A. Schwarz, ${ }^{103}$ Ph. Schwemling, ${ }^{142}$ R. Schwienhorst, ${ }^{104}$ A. Sciandra, ${ }^{24}$ G. Sciolla, ${ }^{26}$ M. Scornajenghi, ${ }^{40 b, 40 a}$ F. Scuri, ${ }^{69 a}$ F. Scutti, ${ }^{102}$ L. M. Scyboz, ${ }^{113}$ C. D. Sebastiani, ${ }^{70 a, 70 b}$ P. Seema, ${ }^{19}$ S. C. Seidel, ${ }^{116}$ A. Seiden, ${ }^{143}$ T. Seiss, ${ }^{36}$ J. M. Seixas ${ }^{78 b}$ G. Sekhniaidze, ${ }^{67 a}$ K. Sekhon, ${ }^{103}$ S. J. Sekula, ${ }^{41}$ N. Semprini-Cesari ${ }^{23 b, 23 a}$ S. Sen, ${ }^{47}$ S. Senkin, ${ }^{37}$ C. Serfon, ${ }^{131}$ L. Serin, ${ }^{129}$ L. Serkin, ${ }^{64 a, 64 b}$ M. Sessa,${ }^{58 a}$ H. Severini, ${ }^{125}$ F. Sforza,${ }^{167}$ A. Sfyrla, ${ }^{52}$ E. Shabalina,${ }^{51}$ J. D. Shahinian, ${ }^{143}$ N. W. Shaikh, ${ }^{43 a, 43 b}$ D. Shaked Renous, ${ }^{177}$ L. Y. Shan, ${ }^{15 a}$ R. Shang, ${ }^{170}$ J. T. Shank, ${ }^{25}$ M. Shapiro, ${ }^{18}$ A. S. Sharma, ${ }^{1}$ A. Sharma, ${ }^{132}$ P. B. Shatalov, ${ }^{109}$ K. Shaw, ${ }^{153}$ S. M. Shaw, ${ }^{98}$ A. Shcherbakova, ${ }^{135}$ Y. Shen, ${ }^{125}$ N. Sherafati, ${ }^{33}$ A. D. Sherman ${ }^{25}$ P. Sherwood,${ }^{92}$ L. Shi, ${ }^{155, \text { rr }}$ S. Shimizu, ${ }^{79}$ C. O. Shimmin,${ }^{180}$ Y. Shimogama, ${ }^{176}$ M. Shimojima, ${ }^{114}$ I. P. J. Shipsey, ${ }^{132}$ S. Shirabe, ${ }^{85}$ M. Shiyakova,${ }^{77}$ J. Shlomi, ${ }^{177}$ A. Shmeleva ${ }^{108}$ D. Shoaleh Saadi, ${ }^{107}$ M. J. Shochet, ${ }^{36}$ S. Shojaii, ${ }^{102}$ D. R. Shope, ${ }^{125}$ S. Shrestha, ${ }^{123}$ E. Shulga, ${ }^{110}$ P. Sicho, ${ }^{138}$ A. M. Sickles, ${ }^{170}$ P. E. Sidebo, ${ }^{151}$ E. Sideras Haddad, ${ }^{32 c}$ O. Sidiropoulou, ${ }^{35}$ A. Sidoti ${ }^{23 b, 23 a}$ F. Siegert, ${ }^{46}$ Dj. Sijacki, ${ }^{16}$ J. Silva, ${ }^{137 a}$ M. Silva Jr., ${ }^{178}$ M. V. Silva Oliveira, ${ }^{78 a}$ S. B. Silverstein, ${ }^{43 a}$ S. Simion, ${ }^{129}$ E. Simioni,${ }^{97}$ M. Simon,${ }^{97}$ R. Simoniello, ${ }^{97}$ P. Sinervo, ${ }^{164}$ N. B. Sinev, ${ }^{128}$ M. Sioli, ${ }^{23 b, 23 a}$ I. Siral, ${ }^{103}$ S. Yu. Sivoklokov, ${ }^{111}$ J. Sjölin, ${ }^{43 a, 43 b}$ P. Skubic, ${ }^{125}$ M. Slater, ${ }^{21}$ T. Slavicek, ${ }^{139}$ M. Slawinska, ${ }^{82}$ K. Sliwa, ${ }^{167}$ R. Slovak, ${ }^{140}$ V. Smakhtin, ${ }^{177}$ B. H. Smart, ${ }^{5}$ J. Smiesko, ${ }^{28 a}$ N. Smirnov, ${ }^{110}$ S. Yu. Smirnov, ${ }^{110}$ Y. Smirnov, ${ }^{110}$ L. N. Smirnova, ${ }^{111}$ O. Smirnova, ${ }^{94}$ J. W. Smith, ${ }^{51}$ M. Smizanska, ${ }^{87}$ K. Smolek, ${ }^{139}$ A. Smykiewicz, ${ }^{82}$ A. A. Snesarev, ${ }^{108}$ I. M. Snyder, ${ }^{128}$ S. Snyder ${ }^{29}$ R. Sobie,${ }^{173,0}$ A. M. Soffa, ${ }^{168}$ A. Soffer, ${ }^{158}$ A. Søgaard, ${ }^{48}$ D. A. Soh, ${ }^{155}$ G. Sokhrannyi, ${ }^{89}$ C. A. Solans Sanchez,${ }^{35}$ M. Solar, ${ }^{139}$ E. Yu. Soldatov ${ }^{110}$ U. Soldevila, ${ }^{171}$ A. A. Solodkov, ${ }^{121}$ A. Soloshenko, ${ }^{77}$ O. V. Solovyanov, ${ }^{121}$ V. Solovyev, ${ }^{135}$ P. Sommer, ${ }^{146}$ H. Son, ${ }^{167}$ W. Song, ${ }^{141}$ W. Y. Song, ${ }^{165 b}$ A. Sopczak, ${ }^{139}$ F. Sopkova, ${ }^{28 b}$ C. L. Sotiropoulou ${ }^{69 a, 69 b}$ S. Sottocornola ${ }^{68 a, 68 b}$ R. Soualah, ${ }^{64 a, 64 c, s s}$ A. M. Soukharev, ${ }^{120 b, 120 a}$ D. South, ${ }^{44}$ B. C. Sowden, ${ }^{11}$ S. Spagnolo, ${ }^{65 a, 65 b}$ M. Spalla, ${ }^{113}$ M. Spangenberg, ${ }^{175}$ F. Spanò,${ }^{91}$ D. Sperlich, ${ }^{19}$ T. M. Spieker, ${ }^{59 a}$ R. Spighi, ${ }^{23 b}$ G. Spigo, ${ }^{35}$ L. A. Spiller, ${ }^{102}$ D. P. Spiteri, ${ }^{55}$ M. Spousta, ${ }^{140}$ A. Stabile,${ }^{66,66 b}$ R. Stamen, ${ }^{59 a}$ S. Stamm, ${ }^{19}$ E. Stanecka, ${ }^{82}$ R. W. Stanek, ${ }^{6}$ C. Stanescu, ${ }^{72 a}$ B. Stanislaus, ${ }^{132}$ M. M. Stanitzki, ${ }^{44}$ B. Stapf, ${ }^{118}$ S. Stapnes, ${ }^{131}$ E. A. Starchenko, ${ }^{121}$ G. H. Stark, ${ }^{36}$ J. Stark,${ }^{56}$ S. H Stark, ${ }^{39}$ P. Staroba,${ }^{138}$ P. Starovoitov, ${ }^{59 a}$ S. Stärz, ${ }^{35}$ R. Staszewski, ${ }^{82}$ M. Stegler, ${ }^{44}$ P. Steinberg, ${ }^{29}$ B. Stelzer, ${ }^{149}$ H. J. Stelzer, ${ }^{35}$ O. Stelzer-Chilton, ${ }^{165 a}$ H. Stenzel,${ }^{54}$ T. J. Stevenson,${ }^{90}$ G. A. Stewart, ${ }^{35}$ M. C. Stockton, ${ }^{35}$ G. Stoicea, ${ }^{27 b}$ P. Stolte, ${ }^{51}$ S. Stonjek, ${ }^{113}$ A. Straessner, ${ }^{46}$ J. Strandberg, ${ }^{151}$ S. Strandberg, ${ }^{43 a, 43 b}$ M. Strauss, ${ }^{125}$ P. Strizenec, ${ }^{28 b}$ R. Ströhmer, ${ }^{174}$ D. M. Strom, ${ }^{128}$ R. Stroynowski, ${ }^{41}$ A. Strubig, ${ }^{48}$ S. A. Stucci, ${ }^{29}$ B. Stugu, ${ }^{17}$ J. Stupak, ${ }^{125}$ N. A. Styles, ${ }^{44}$ D. Su, ${ }^{150}$ J. Su, ${ }^{136}$ S. Suchek, ${ }^{59 a}$ Y. Sugaya,${ }^{130}$ M. Suk,${ }^{139}$ V. V. Sulin, ${ }^{108}$ M. J. Sullivan ${ }^{88}$ D. M. S. Sultan, ${ }^{52}$ S. Sultansoy, ${ }^{4 c}$ T. Sumida, ${ }^{83}$ S. Sun, ${ }^{103}$ X. Sun, ${ }^{3}$ K. Suruliz, ${ }^{153}$ C. J. E. Suster, ${ }^{154}$ M. R. Sutton, ${ }^{153}$ S. Suzuki, ${ }^{79}$ M. Svatos, ${ }^{138}$ M. Swiatlowski,${ }^{36}$ S. P. Swift, ${ }^{2}$ A. Sydorenko, ${ }^{97}$ I. Sykora, ${ }^{28 a}$ T. Sykora, ${ }^{140}$ D. Ta, ${ }^{97}$ K. Tackmann,,${ }^{44, t t}$ J. Taenzer, ${ }^{158}$ A. Taffard,${ }^{168}$ R. Tafirout, ${ }^{165 a}$ E. Tahirovic, ${ }^{90}$ N. Taiblum, ${ }^{158}$ H. Takai, ${ }^{29}$ R. Takashima, ${ }^{84}$ E. H. Takasugi, ${ }^{113}$ K. Takeda, ${ }^{80}$ T. Takeshita, ${ }^{147}$ Y. Takubo, ${ }^{79}$ M. Talby, ${ }^{99}$ A. A. Talyshev, ${ }^{120 b, 120 a}$ J. Tanaka, ${ }^{160}$ M. Tanaka ${ }^{162}$ R. Tanaka,${ }^{129}$ B. B. Tannenwald, ${ }^{123}$ S. Tapia Araya, ${ }^{144 \mathrm{~b}}$ S. Tapprogge, ${ }^{97}$

A. Tarek Abouelfadl Mohamed, ${ }^{133}$ S. Tarem, ${ }^{157}$ G. Tarna, ${ }^{27 b, r}$ G. F. Tartarelli, ${ }^{66 a}$ P. Tas, ${ }^{140}$ M. Tasevsky, ${ }^{138}$ T. Tashiro, ${ }^{83}$ E. Tassi, ${ }^{40 b, 40 a}$ A. Tavares Delgado, ${ }^{137 a, 137 b}$ Y. Tayalati, ${ }^{34 \mathrm{e}}$ A. C. Taylor, ${ }^{116}$ A. J. Taylor, ${ }^{48}$ G. N. Taylor, ${ }^{102}$ P. T. E. Taylor, ${ }^{102}$ W. Taylor ${ }^{165 b}$ A. S. Tee,${ }^{87}$ R. Teixeira De Lima, ${ }^{150}$ P. Teixeira-Dias, ${ }^{91}$ H. Ten Kate,${ }^{35}$ J. J. Teoh, ${ }^{118}$ S. Terada,${ }^{79}$ K. Terashi, ${ }^{160}$ J. Terron, ${ }^{96}$ S. Terzo, ${ }^{14}$ M. Testa, ${ }^{49}$ R. J. Teuscher, ${ }^{164,0}$ S. J. Thais, ${ }^{180}$ T. Theveneaux-Pelzer, ${ }^{44}$ F. Thiele, ${ }^{39}$ D. W. Thomas, ${ }^{91}$ J. P. Thomas, ${ }^{21}$ A. S. Thompson, ${ }^{55}$ P. D. Thompson, ${ }^{21}$ L. A. Thomsen, ${ }^{180}$ E. Thomson, ${ }^{134}$ Y. Tian, ${ }^{38}$ R. E. Ticse Torres,${ }^{51}$ V. O. Tikhomirov, ${ }^{108, \text { uu }}$ Yu. A. Tikhonov, ${ }^{120 b, 120 \mathrm{a}}$ S. Timoshenko, ${ }^{110} \mathrm{P}$. Tipton, ${ }^{180} \mathrm{~S}$. Tisserant, ${ }^{99} \mathrm{~K}$. Todome, ${ }^{162}$

S. Todorova-Nova, ${ }^{5}$ S. Todt, ${ }^{46}$ J. Tojo, ${ }^{85}$ S. Tokár, ${ }^{28 a}$ K. Tokushuku, ${ }^{79}$ E. Tolley,${ }^{123}$ K. G. Tomiwa, ${ }^{32 c}$ M. Tomoto, ${ }^{115}$ L. Tompkins, ${ }^{150, g g}$ K. Toms, ${ }^{116}$ B. Tong,${ }^{57}$ P. Tornambe,${ }^{50}$ E. Torrence, ${ }^{128}$ H. Torres ${ }^{46}$ E. Torró Pastor, ${ }^{145}$ C. Tosciri, ${ }^{132}$ J. Toth, ${ }^{99, v v}$ F. Touchard, ${ }^{99}$ D. R. Tovey, ${ }^{146}$ C. J. Treado, ${ }^{122}$ T. Trefzger, ${ }^{174}$ F. Tresoldi, ${ }^{153}$ A. Tricoli, ${ }^{29}$ I. M. Trigger, ${ }^{165 a}$ 
S. Trincaz-Duvoid ${ }^{133}$ M. F. Tripiana, ${ }^{14}$ W. Trischuk, ${ }^{164}$ B. Trocmé,${ }^{56}$ A. Trofymov, ${ }^{129}$ C. Troncon, ${ }^{66 a}$ M. Trovatelli, ${ }^{173}$ F. Trovato, ${ }^{153}$ L. Truong, ${ }^{32 b}$ M. Trzebinski, ${ }^{82}$ A. Trzupek, ${ }^{82}$ F. Tsai,${ }^{44}$ J. C-L. Tseng, ${ }^{132}$ P. V. Tsiareshka, ${ }^{105, i i}$ A. Tsirigotis, ${ }^{159}$ N. Tsirintanis,${ }^{9}$ V. Tsiskaridze, ${ }^{152}$ E. G. Tskhadadze, ${ }^{156 a}$ I. I. Tsukerman, ${ }^{109}$ V. Tsulaia,${ }^{18}$ S. Tsuno,${ }^{79}$ D. Tsybychev,${ }^{152,163}$ Y. Tu, ${ }^{61 \mathrm{~b}}$ A. Tudorache, ${ }^{27 \mathrm{~b}}$ V. Tudorache, ${ }^{27 \mathrm{~b}}$ T. T. Tulbure, ${ }^{27 \mathrm{a}}$ A. N. Tuna, ${ }^{57}$ S. Turchikhin, ${ }^{77}$ D. Turgeman, ${ }^{177}$ I. Turk Cakir, ${ }^{4 b, w w}$ R. Turra, ${ }^{66 a}$ P. M. Tuts, ${ }^{38}$ E. Tzovara, ${ }^{97}$ G. Ucchielli, ${ }^{45}$ I. Ueda ${ }^{79}$ M. Ughetto,${ }^{43 a, 43 b}$ F. Ukegawa, ${ }^{166}$ G. Unal, ${ }^{35}$ A. Undrus, ${ }^{29}$ G. Unel, ${ }^{168}$ F. C. Ungaro, ${ }^{102}$ Y. Unno, ${ }^{79}$ K. Uno, ${ }^{160}$ J. Urban,${ }^{28 b}$ P. Urquijo, ${ }^{102}$ P. Urrejola,${ }^{97}$ G. Usai ${ }^{8}$ J. Usui ${ }^{79}$ L. Vacavant, ${ }^{99}$ V. Vacek,${ }^{139}$ B. Vachon, ${ }^{101}$ K. O. H. Vadla, ${ }^{131}$ A. Vaidya, ${ }^{92}$ C. Valderanis, ${ }^{112}$ E. Valdes Santurio, ${ }^{43 a, 43 b}$ M. Valente, ${ }^{52}$ S. Valentinetti, ${ }^{23 b, 23 a}$ A. Valero, ${ }^{171}$ L. Valéry, ${ }^{44}$ R. A. Vallance, ${ }^{21}$ A. Vallier, ${ }^{5}$ J. A. Valls Ferrer, ${ }^{171}$ T. R. Van Daalen ${ }^{14}$ H. Van der Graaf, ${ }^{118}$ P. Van Gemmeren, ${ }^{6}$ I. Van Vulpen, ${ }^{118}$ M. Vanadia, ${ }^{71 a, 71 b}$ W. Vandelli, ${ }^{35}$ A. Vaniachine, ${ }^{163}$ P. Vankov, ${ }^{118}$ R. Vari, ${ }^{70 a}$ E. W. Varnes, ${ }^{7}$ C. Varni, ${ }^{53 b, 53 a}$ T. Varol,,${ }^{41}$ D. Varouchas, ${ }^{129}$ K. E. Varvell, ${ }^{154}$ G. A. Vasquez, ${ }^{144 b}$ J. G. Vasquez, ${ }^{180}$ F. Vazeille, ${ }^{37}$ D. Vazquez Furelos, ${ }^{14}$ T. Vazquez Schroeder,${ }^{35}$ J. Veatch, ${ }^{51}$ V. Vecchio, ${ }^{72 a, 72 b}$ L. M. Veloce, ${ }^{164}$ F. Veloso, ${ }^{137 a, 137 c}$ S. Veneziano, ${ }^{70 a}$ A. Ventura, ${ }^{65 a, 65 b}$ N. Venturi, ${ }^{35}$ V. Vercesi, ${ }^{68 \mathrm{a}}$ M. Verducci, ${ }^{72 a, 72 b}$ C. M. Vergel Infante, ${ }^{76}$ C. Vergis, ${ }^{24}$ W. Verkerke ${ }^{118}$ A. T. Vermeulen, ${ }^{118}$ J. C. Vermeulen, ${ }^{118}$ M. C. Vetterli, ${ }^{149, f}$ N. Viaux Maira, ${ }^{144 b}$ M. Vicente Barreto Pinto, ${ }^{52}$ I. Vichou, ${ }^{170, a}$ T. Vickey, ${ }^{146}$ O. E. Vickey Boeriu, ${ }^{146}$ G. H. A. Viehhauser, ${ }^{132}$ S. Viel, ${ }^{18}$ L. Vigani, ${ }^{132}$ M. Villa, ${ }^{23 b, 23 a}$ M. Villaplana Perez, ${ }^{66 a, 66 b}$ E. Vilucchi, ${ }^{49}$ M. G. Vincter, ${ }^{33}$ V. B. Vinogradov, ${ }^{77}$ A. Vishwakarma, ${ }^{44}$ C. Vittori, ${ }^{23 b, 23 a}$ I. Vivarelli, ${ }^{153}$ S. Vlachos,${ }^{10}$ M. Vogel, ${ }^{179}$ P. Vokac, ${ }^{139}$ G. Volpi,${ }^{14}$ S. E. von Buddenbrock, ${ }^{32 c}$ E. Von Toerne, ${ }^{24}$ V. Vorobel,,${ }^{140}$ K. Vorobev,${ }^{110}$ M. Vos, ${ }^{171}$ J. H. Vossebeld, ${ }^{88}$ N. Vranjes, ${ }^{16}$ M. Vranjes Milosavljevic, ${ }^{16}$ V. Vrba, ${ }^{139}$ M. Vreeswijk, ${ }^{118}$ T. Šfiligoj, ${ }^{89}$ R. Vuillermet,${ }^{35}$ I. Vukotic ${ }^{36}$ T. Ženišs, ${ }^{28 a}$ L. Živković, ${ }^{16}$ P. Wagner, ${ }^{24}$ W. Wagner, ${ }^{179}$ J. Wagner-Kuhr, ${ }^{112}$ H. Wahlberg, ${ }^{86}$ S. Wahrmund ${ }^{46}$ K. Wakamiya, ${ }^{80}$ V. M. Walbrecht, ${ }^{113}$ J. Walder, ${ }^{87}$ R. Walker, ${ }^{112}$ S. D. Walker, ${ }^{91}$ W. Walkowiak, ${ }^{148}$ V. Wallangen, ${ }^{43 a, 43 b}$ A. M. Wang, ${ }^{57}$ C. Wang, ${ }^{58 b}$ F. Wang, ${ }^{178}$ H. Wang, ${ }^{18}$ H. Wang, ${ }^{3}$ J. Wang,${ }^{154}$ J. Wang,${ }^{59 b}$ P. Wang, ${ }^{41}$ Q. Wang, ${ }^{125}$ R.-J. Wang, ${ }^{133}$ R. Wang, ${ }^{58 a}$ R. Wang, ${ }^{6}$ S. M. Wang, ${ }^{155}$ W. T. Wang, ${ }^{58 a}$ W. Wang, ${ }^{15 c, x x}$ W. X. Wang, ${ }^{58 a, x x}$ Y. Wang, ${ }^{58 a, k k}$ Z. Wang, ${ }^{58 c}$ C. Wanotayaroj, ${ }^{44}$ A. Warburton, ${ }^{101}$ C. P. Ward, ${ }^{31}$ D. R. Wardrope, ${ }^{92}$ A. Washbrook, ${ }^{48}$ P. M. Watkins, ${ }^{21}$ A. T. Watson, ${ }^{21}$ M. F. Watson, ${ }^{21}$ G. Watts, ${ }^{145}$ S. Watts ${ }^{98}$ B. M. Waugh, ${ }^{92}$ A. F. Webb ${ }^{11}$ S. Webb, ${ }^{97}$ C. Weber, ${ }^{180}$ M. S. Weber, ${ }^{20}$ S. A. Weber, ${ }^{33}$ S. M. Weber, ${ }^{59 a}$ A. R. Weidberg, ${ }^{132}$ J. Weingarten,${ }^{45}$ M. Weirich,${ }^{97}$ C. Weiser, ${ }^{50}$ P. S. Wells,${ }^{35}$ T. Wenaus, ${ }^{29}$ T. Wengler, ${ }^{35}$ S. Wenig, ${ }^{35}$ N. Wermes, ${ }^{24}$ M. D. Werner,${ }^{76}$ P. Werner,${ }^{35}$ M. Wessels, ${ }^{59 a}$ T. D. Weston, ${ }^{20}$ K. Whalen, ${ }^{128}$ N. L. Whallon, ${ }^{145}$ A. M. Wharton ${ }^{87}$ A. S. White, ${ }^{103}$ A. White, ${ }^{8}$ M. J. White, ${ }^{1}$ R. White, ${ }^{144 b}$ D. Whiteson, ${ }^{168}$ B. W. Whitmore, ${ }^{87}$ F. J. Wickens, ${ }^{141}$ W. Wiedenmann, ${ }^{178}$ M. Wielers, ${ }^{141}$ C. Wiglesworth, ${ }^{39}$ L. A. M. Wiik-Fuchs, ${ }^{50}$ F. Wilk, ${ }^{98}$ H. G. Wilkens, ${ }^{35}$ L. J. Wilkins, ${ }^{91}$ H. H. Williams, ${ }^{134}$ S. Williams,${ }^{31}$ C. Willis, ${ }^{104}$ S. Willocq,${ }^{100}$ J. A. Wilson, ${ }^{21}$ I. Wingerter-Seez, ${ }^{5}$ E. Winkels, ${ }^{153}$ F. Winklmeier, ${ }^{128}$ O. J. Winston, ${ }^{153}$ B. T. Winter, ${ }^{50}$ M. Wittgen, ${ }^{150}$ M. Wobisch, ${ }^{93}$

A. Wolf, ${ }^{97}$ T. M. H. Wolf, ${ }^{118}$ R. Wolff, ${ }^{99}$ M. W. Wolter, ${ }^{82}$ H. Wolters, ${ }^{137 a, 137 c}$ V. W. S. Wong, ${ }^{172}$ N. L. Woods, ${ }^{143}$ S. D. Worm, ${ }^{21}$ B. K. Wosiek, ${ }^{82}$ K. W. Woźniak, ${ }^{82}$ K. Wraight, ${ }^{55}$ M. Wu ${ }^{36}$ S. L. Wu, ${ }^{178}$ X. Wu, ${ }^{52}$ Y. Wu, ${ }^{58 a}$ T. R. Wyatt, ${ }^{98}$ B. M. Wynne, ${ }^{48}$ S. Xella, ${ }^{39}$ Z. Xi ${ }^{103}$ L. Xia, ${ }^{175}$ D. Xu, ${ }^{15 a}$ H. Xu ${ }^{58 a, r}$ L. Xu ${ }^{29}$ T. Xu, ${ }^{142}$ W. Xu, ${ }^{103}$ Z. Xu, ${ }^{150}$ B. Yabsley, ${ }^{154}$ S. Yacoob, ${ }^{32 a}$ K. Yajima, ${ }^{130}$ D. P. Yallup, ${ }^{92}$ D. Yamaguchi, ${ }^{162}$ Y. Yamaguchi, ${ }^{162}$ A. Yamamoto, ${ }^{79}$ T. Yamanaka, ${ }^{160}$ F. Yamane ${ }^{80}$ M. Yamatani, ${ }^{160}$ T. Yamazaki ${ }^{160}$ Y. Yamazaki, ${ }^{80}$ Z. Yan, ${ }^{25}$ H. J. Yang, ${ }^{58 c, 58 d}$ H. T. Yang, ${ }^{18}$ S. Yang, ${ }^{75}$ Y. Yang, ${ }^{160}$ Z. Yang, ${ }^{17}$ W-M. Yao, ${ }^{18}$ Y. C. Yap, ${ }^{44}$ Y. Yasu, ${ }^{79}$ E. Yatsenko, ${ }^{58 c, 58 d}$ J. Ye, ${ }^{41}$ S. Ye, ${ }^{29}$ I. Yeletskikh, ${ }^{77}$ E. Yigitbasi, ${ }^{25}$ E. Yildirim, ${ }^{97}$ K. Yorita, ${ }^{176}$ K. Yoshihara, ${ }^{134}$ C. J. S. Young, ${ }^{35}$ C. Young, ${ }^{150}$ J. Yu, ${ }^{8}$ J. Yu ${ }^{76}$ X. Yue, ${ }^{59 a}$ S. P. Y. Yuen, ${ }^{24}$ B. Zabinski, ${ }^{82}$ G. Zacharis, ${ }^{10}$ E. Zaffaroni, ${ }^{52}$ R. Zaidan, ${ }^{14}$ A. M. Zaitsevv ${ }^{121,00}$ T. Zakareishvili, ${ }^{156 b}$ N. Zakharchuk ${ }^{33}$ J. Zalieckas, ${ }^{17}$ S. Zambito, ${ }^{57}$ D. Zanzi, ${ }^{35}$ D. R. Zaripovas, ${ }^{55}$ S. V. Zeißner, ${ }^{45}$ C. Zeitnitz, ${ }^{179}$ G. Zemaityte, ${ }^{132}$ J. C. Zeng, ${ }^{170}$ Q. Zeng, ${ }^{150}$ O. Zenin, ${ }^{121}$ D. Zerwas, ${ }^{129}$ M. Zgubič ${ }^{132}$ D. F. Zhang, ${ }^{58 b}$ D. Zhang, ${ }^{103}$ F. Zhang, ${ }^{178}$ G. Zhang, ${ }^{58 \mathrm{a}}$ G. Zhang, ${ }^{15 \mathrm{~b}}$ H. Zhang, ${ }^{15 \mathrm{c}}$ J. Zhang, ${ }^{6}$ L. Zhang, ${ }^{15 \mathrm{c}}$ L. Zhang, ${ }^{58 \mathrm{a}}$ M. Zhang, ${ }^{170}$ P. Zhang, ${ }^{15 \mathrm{c}}$ R. Zhang, ${ }^{58 \mathrm{a}}$ R. Zhang, ${ }^{24}$ X. Zhang, ${ }^{58 \mathrm{~b}}$ Y. Zhang, ${ }^{15 \mathrm{~d}}$ Z. Zhang, ${ }^{129}$ P. Zhao, ${ }^{47}$ Y. Zhao, ${ }^{58 \mathrm{~b}, 129, \text { aa }}$ Z. Zhao, ${ }^{58 \mathrm{a}}$ A. Zhemchugov, ${ }^{77}$ Z. Zheng, ${ }^{103}$ D. Zhong, ${ }^{170}$ B. Zhou, ${ }^{103}$ C. Zhou ${ }^{178}$ L. Zhou, ${ }^{41}$ M. S. Zhou, ${ }^{15 d}$ M. Zhou, ${ }^{152}$ N. Zhou, ${ }^{58 c}$ Y. Zhou, ${ }^{7}$ C. G. Zhu, ${ }^{58 b}$ H. L. Zhu, ${ }^{58 a}$ H. Zhu, ${ }^{15 a}$ J. Zhu, ${ }^{103}$ Y. Zhu, ${ }^{58 \mathrm{a}}$ X. Zhuang, ${ }^{15 \mathrm{a}} \mathrm{K}$. Zhukov, ${ }^{108}$ V. Zhulanov, ${ }^{120 b, 120 \mathrm{a}}$ A. Zibell, ${ }^{174}$ D. Zieminska, ${ }^{63}$ N. I. Zimine, ${ }^{77}$ S. Zimmermann, ${ }^{50}$ Z. Zinonos, ${ }^{113}$ M. Ziolkowski, ${ }^{148}$ G. Zobernig, ${ }^{178}$ A. Zoccoli, ${ }^{23 b, 23 a}$ K. Zoch, ${ }^{51}$ T. G. Zorbas, ${ }^{146}$ R. Zou, ${ }^{36}$ M. Zur Nedden, ${ }^{19}$ and L. Zwalinski ${ }^{35}$

(ATLAS Collaboration) 
${ }^{1}$ Department of Physics, University of Adelaide, Adelaide, Australia

${ }^{2}$ Physics Department, SUNY Albany, Albany, New York, USA

${ }^{3}$ Department of Physics, University of Alberta, Edmonton, Alberta, Canada

${ }^{4 \mathrm{a}}$ Department of Physics, Ankara University, Ankara, Turkey

${ }^{4 \mathrm{~b}}$ Istanbul Aydin University, Istanbul, Turkey

${ }^{4 \mathrm{c}}$ Division of Physics, TOBB University of Economics and Technology, Ankara, Turkey

${ }^{5}$ LAPP, Université Grenoble Alpes, Université Savoie Mont Blanc, CNRS/IN2P3, Annecy, France

${ }^{6}$ High Energy Physics Division, Argonne National Laboratory, Argonne, Illinois, USA

${ }^{7}$ Department of Physics, University of Arizona, Tucson, Arizona, USA

${ }^{8}$ Department of Physics, University of Texas at Arlington, Arlington, Texas, USA

${ }^{9}$ Physics Department, National and Kapodistrian University of Athens, Athens, Greece

${ }^{10}$ Physics Department, National Technical University of Athens, Zografou, Greece

${ }^{11}$ Department of Physics, University of Texas at Austin, Austin, Texas, USA

${ }^{12 a}$ Bahcesehir University, Faculty of Engineering and Natural Sciences, Istanbul, Turkey

${ }^{12 \mathrm{~b}}$ Istanbul Bilgi University, Faculty of Engineering and Natural Sciences, Istanbul, Turkey

${ }^{12 \mathrm{c}}$ Department of Physics, Bogazici University, Istanbul, Turkey

${ }^{12 \mathrm{~d}}$ Department of Physics Engineering, Gaziantep University, Gaziantep, Turkey

${ }^{13}$ Institute of Physics, Azerbaijan Academy of Sciences, Baku, Azerbaijan

${ }^{14}$ Institut de Física d'Altes Energies (IFAE), Barcelona Institute of Science and Technology, Barcelona, Spain

${ }^{15 a}$ Institute of High Energy Physics, Chinese Academy of Sciences, Beijing, China

${ }^{15 \mathrm{~b}}$ Physics Department, Tsinghua University, Beijing, China

${ }^{15 \mathrm{c}}$ Department of Physics, Nanjing University, Nanjing, China

${ }^{15 \mathrm{~d}}$ University of Chinese Academy of Science (UCAS), Beijing, China

${ }^{16}$ Institute of Physics, University of Belgrade, Belgrade, Serbia

${ }^{17}$ Department for Physics and Technology, University of Bergen, Bergen, Norway

${ }^{18}$ Physics Division, Lawrence Berkeley National Laboratory and University of California, Berkeley, California, USA

${ }^{19}$ Institut für Physik, Humboldt Universität zu Berlin, Berlin, Germany

${ }^{20}$ Albert Einstein Center for Fundamental Physics and Laboratory for High Energy Physics, University of Bern, Bern, Switzerland

${ }^{21}$ School of Physics and Astronomy, University of Birmingham, Birmingham, United Kingdom

${ }^{22}$ Centro de Investigaciónes, Universidad Antonio Nariño, Bogota, Colombia

${ }^{23 a}$ Dipartimento di Fisica e Astronomia, Università di Bologna, Bologna, Italy

${ }^{23 \mathrm{~b}}$ INFN Sezione di Bologna, Italy

${ }^{24}$ Physikalisches Institut, Universität Bonn, Bonn, Germany

${ }^{25}$ Department of Physics, Boston University, Boston, Massachusetts, USA

${ }^{26}$ Department of Physics, Brandeis University, Waltham, Massachusetts, USA

${ }^{27 a}$ Transilvania University of Brasov, Brasov, Romania

${ }^{27 \mathrm{~b}}$ Horia Hulubei National Institute of Physics and Nuclear Engineering, Bucharest, Romania

${ }^{27 \mathrm{c}}$ Department of Physics, Alexandru Ioan Cuza University of Iasi, Iasi, Romania

${ }^{27 \mathrm{~d}}$ National Institute for Research and Development of Isotopic and Molecular Technologies,

Physics Department, Cluj-Napoca, Romania

${ }^{27 \mathrm{e}}$ University Politehnica Bucharest, Bucharest, Romania

${ }^{27 f}$ West University in Timisoara, Timisoara, Romania

${ }^{28 a}$ Faculty of Mathematics, Physics and Informatics, Comenius University, Bratislava, Slovak Republic

${ }^{28 \mathrm{~b}}$ Department of Subnuclear Physics, Institute of Experimental Physics of the Slovak Academy of Sciences, Kosice, Slovak Republic

${ }^{29}$ Physics Department, Brookhaven National Laboratory, Upton, New York, USA

${ }^{30}$ Departamento de Física, Universidad de Buenos Aires, Buenos Aires, Argentina

${ }^{31}$ Cavendish Laboratory, University of Cambridge, Cambridge, United Kingdom

${ }^{32 a}$ Department of Physics, University of Cape Town, Cape Town, South Africa

${ }^{32 \mathrm{~b}}$ Department of Mechanical Engineering Science, University of Johannesburg, Johannesburg, South Africa

${ }^{32 \mathrm{c} S c h o o l}$ of Physics, University of the Witwatersrand, Johannesburg, South Africa

${ }^{33}$ Department of Physics, Carleton University, Ottawa, Ontario, Canada

${ }^{34 a}$ Faculté des Sciences Ain Chock, Réseau Universitaire de Physique des Hautes Energies-Université Hassan II, Casablanca, Morocco

${ }^{34 \mathrm{~b}}$ Centre National de l'Energie des Sciences Techniques Nucleaires (CNESTEN), Rabat, Morocco

${ }^{34 \mathrm{c}}$ Faculté des Sciences Semlalia, Université Cadi Ayyad, LPHEA-Marrakech, Morocco

${ }^{34 \mathrm{~d}}$ Faculté des Sciences, Université Mohamed Premier and LPTPM, Oujda, Morocco

${ }^{34}$ Faculté des sciences, Université Mohammed V, Rabat, Morocco

${ }^{35}$ CERN, Geneva, Switzerland

${ }^{36}$ Enrico Fermi Institute, University of Chicago, Chicago, Illinois, USA 


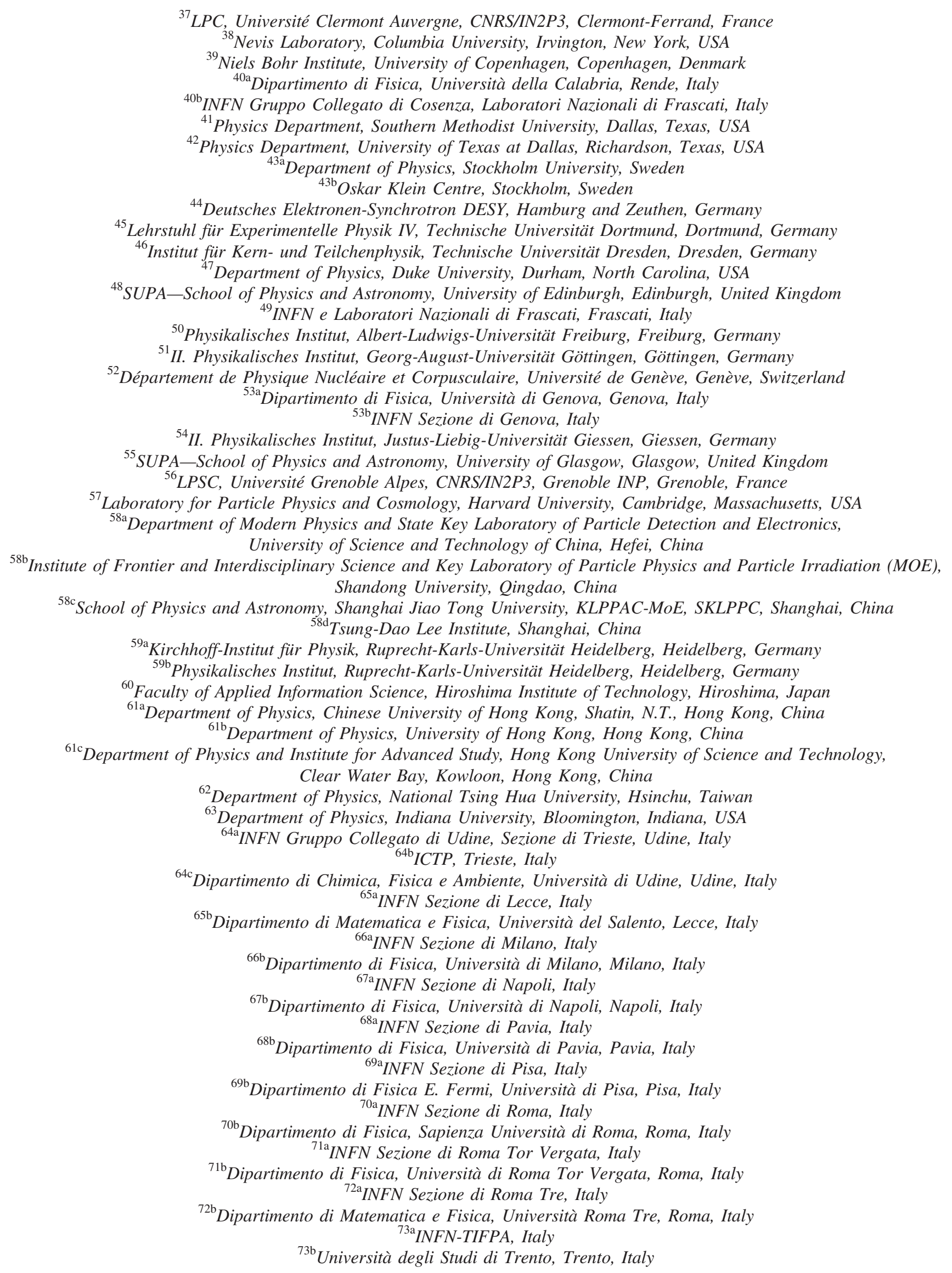




\footnotetext{
${ }^{74}$ Institut für Astro- und Teilchenphysik, Leopold-Franzens-Universität, Innsbruck, Austria

${ }^{75}$ University of Iowa, Iowa City, Iowa, USA

${ }^{76}$ Department of Physics and Astronomy, Iowa State University, Ames, Iowa, USA

${ }^{77}$ Joint Institute for Nuclear Research, Dubna, Russia

${ }^{78 a}$ Departamento de Engenharia Elétrica, Universidade Federal de Juiz de Fora (UFJF), Juiz de Fora, Brazil

${ }^{78 b}$ Universidade Federal do Rio De Janeiro COPPE/EE/IF, Rio de Janeiro, Brazil

${ }^{78 c}$ Universidade Federal de São João del Rei (UFSJ), São João del Rei, Brazil

${ }^{78 d}$ Instituto de Física, Universidade de São Paulo, São Paulo, Brazil

${ }^{79}$ KEK, High Energy Accelerator Research Organization, Tsukuba, Japan

${ }^{80}$ Graduate School of Science, Kobe University, Kobe, Japan

${ }^{81 \mathrm{a}}$ AGH University of Science and Technology, Faculty of Physics and Applied Computer Science, Krakow, Poland

${ }^{81 \mathrm{~b}}$ Marian Smoluchowski Institute of Physics, Jagiellonian University, Krakow, Poland

${ }^{82}$ Institute of Nuclear Physics Polish Academy of Sciences, Krakow, Poland

${ }^{83}$ Faculty of Science, Kyoto University, Kyoto, Japan

${ }^{84}$ Kyoto University of Education, Kyoto, Japan

${ }^{85}$ Research Center for Advanced Particle Physics and Department of Physics, Kyushu University, Fukuoka, Japan

${ }^{86}$ Instituto de Física La Plata, Universidad Nacional de La Plata and CONICET, La Plata, Argentina

${ }^{87}$ Physics Department, Lancaster University, Lancaster, United Kingdom

${ }^{88}$ Oliver Lodge Laboratory, University of Liverpool, Liverpool, United Kingdom

${ }^{89}$ Department of Experimental Particle Physics, Jožef Stefan Institute and Department of Physics, University of Ljubljana, Ljubljana, Slovenia

${ }^{90}$ School of Physics and Astronomy, Queen Mary University of London, London, United Kingdom

${ }^{91}$ Department of Physics, Royal Holloway University of London, Egham, United Kingdom

${ }^{92}$ Department of Physics and Astronomy, University College London, London, United Kingdom

${ }^{93}$ Louisiana Tech University, Ruston, Louisiana, USA

${ }^{94}$ Fysiska institutionen, Lunds universitet, Lund, Sweden

${ }^{95}$ Centre de Calcul de l'Institut National de Physique Nucléaire et de Physique des Particules (IN2P3), Villeurbanne, France

${ }^{96}$ Departamento de Física Teorica C-15 and CIAFF, Universidad Autónoma de Madrid, Madrid, Spain

${ }^{97}$ Institut für Physik, Universität Mainz, Mainz, Germany

${ }^{98}$ School of Physics and Astronomy, University of Manchester, Manchester, United Kingdom

${ }^{99}$ CPPM, Aix-Marseille Université, CNRS/IN2P3, Marseille, France

${ }^{100}$ Department of Physics, University of Massachusetts, Amherst, Massachusetts, USA

${ }^{101}$ Department of Physics, McGill University, Montreal, Quebec, Canada

${ }^{102}$ School of Physics, University of Melbourne, Victoria, Australia

${ }^{103}$ Department of Physics, University of Michigan, Ann Arbor, Michigan, USA

${ }^{104}$ Department of Physics and Astronomy, Michigan State University, East Lansing, Michigan, USA

${ }^{105}$ B.I. Stepanov Institute of Physics, National Academy of Sciences of Belarus, Minsk, Belarus

${ }^{106}$ Research Institute for Nuclear Problems of Byelorussian State University, Minsk, Belarus

${ }^{107}$ Group of Particle Physics, University of Montreal, Montreal, Quebec, Canada

${ }^{108}$ P.N. Lebedev Physical Institute of the Russian Academy of Sciences, Moscow, Russia

${ }^{109}$ Institute for Theoretical and Experimental Physics (ITEP), Moscow, Russia

${ }^{110}$ National Research Nuclear University MEPhI, Moscow, Russia

${ }^{111}$ D.V. Skobeltsyn Institute of Nuclear Physics, M.V. Lomonosov Moscow State University, Moscow, Russia

${ }^{112}$ Fakultät für Physik, Ludwig-Maximilians-Universität München, München, Germany

${ }^{113}$ Max-Planck-Institut für Physik (Werner-Heisenberg-Institut), München, Germany

${ }^{114}$ Nagasaki Institute of Applied Science, Nagasaki, Japan

${ }^{115}$ Graduate School of Science and Kobayashi-Maskawa Institute, Nagoya University, Nagoya, Japan

${ }^{116}$ Department of Physics and Astronomy, University of New Mexico, Albuquerque, New Mexico, USA

${ }^{117}$ Institute for Mathematics, Astrophysics and Particle Physics, Radboud University Nijmegen/Nikhef, Nijmegen, Netherlands

${ }^{118}$ Nikhef National Institute for Subatomic Physics and University of Amsterdam, Amsterdam, Netherlands

${ }^{119}$ Department of Physics, Northern Illinois University, DeKalb, Illinois, USA

${ }^{120 a}$ Budker Institute of Nuclear Physics and NSU, SB RAS, Novosibirsk, Russia

${ }^{120 \mathrm{~b}}$ Novosibirsk State University Novosibirsk, Russia

${ }^{121}$ Institute for High Energy Physics of the National Research Centre Kurchatov Institute, Protvino, Russia

${ }^{122}$ Department of Physics, New York University, New York, New York, USA

${ }^{123}$ Ohio State University, Columbus, Ohio, USA

${ }^{124}$ Faculty of Science, Okayama University, Okayama, Japan

${ }^{125}$ Homer L. Dodge Department of Physics and Astronomy, University of Oklahoma, Norman, Oklahoma, USA

${ }^{126}$ Department of Physics, Oklahoma State University, Stillwater, Oklahoma, USA

${ }^{127}$ Palacky University, RCPTM, Joint Laboratory of Optics, Olomouc, Czech Republic
} 
${ }^{128}$ Center for High Energy Physics, University of Oregon, Eugene, Oregon, USA

${ }^{129}$ LAL, Université Paris-Sud, CNRS/IN2P3, Université Paris-Saclay, Orsay, France

${ }^{130}$ Graduate School of Science, Osaka University, Osaka, Japan

${ }^{131}$ Department of Physics, University of Oslo, Oslo, Norway

${ }^{132}$ Department of Physics, Oxford University, Oxford, United Kingdom

${ }^{133}$ LPNHE, Sorbonne Université, Paris Diderot Sorbonne Paris Cité, CNRS/IN2P3, Paris, France

${ }^{134}$ Department of Physics, University of Pennsylvania, Philadelphia, Pennsylvania, USA

${ }^{135}$ Konstantinov Nuclear Physics Institute of National Research Centre "Kurchatov Institute”, PNPI, St. Petersburg, Russia

${ }^{136}$ Department of Physics and Astronomy, University of Pittsburgh, Pittsburgh, Pennsylvania, USA

${ }^{137 a}$ Laboratório de Instrumentação e Física Experimental de Partículas-LIP, Portugal

${ }^{137 b}$ Departamento de Física, Faculdade de Ciências, Universidade de Lisboa, Lisboa, Portugal

${ }^{137 \mathrm{c}}$ Departamento de Física, Universidade de Coimbra, Coimbra, Portugal

${ }^{137 \mathrm{~d}}$ Centro de Física Nuclear da Universidade de Lisboa, Lisboa, Portugal

${ }^{137 \mathrm{e}}$ Departamento de Física, Universidade do Minho, Braga, Portugal

${ }^{137 \mathrm{f}}$ Departamento de Física Teorica y del Cosmos, Universidad de Granada, Granada (Spain), Spain

${ }^{137 \mathrm{~g}}$ Dep Física and CEFITEC of Faculdade de Ciências e Tecnologia, Universidade Nova de Lisboa, Caparica, Portugal

${ }^{138}$ Institute of Physics, Academy of Sciences of the Czech Republic, Prague, Czech Republic

${ }^{139}$ Czech Technical University in Prague, Prague, Czech Republic

${ }^{140}$ Charles University, Faculty of Mathematics and Physics, Prague, Czech Republic

${ }^{141}$ Particle Physics Department, Rutherford Appleton Laboratory, Didcot, United Kingdom

${ }^{142}$ IRFU, CEA, Université Paris-Saclay, Gif-sur-Yvette, France

${ }^{143}$ Santa Cruz Institute for Particle Physics, University of California Santa Cruz, Santa Cruz, California, USA

${ }^{144 a}$ Departamento de Física, Pontificia Universidad Católica de Chile, Santiago, Chile

${ }^{144 b}$ Departamento de Física, Universidad Técnica Federico Santa María, Valparaíso, Chile

${ }^{145}$ Department of Physics, University of Washington, Seattle, Washington, USA

${ }^{146}$ Department of Physics and Astronomy, University of Sheffield, Sheffield, United Kingdom

${ }^{147}$ Department of Physics, Shinshu University, Nagano, Japan

${ }^{148}$ Department Physik, Universität Siegen, Siegen, Germany

${ }^{149}$ Department of Physics, Simon Fraser University, Burnaby, British Columbia, Canada

${ }^{150}$ SLAC National Accelerator Laboratory, Stanford, California, USA

${ }^{151}$ Physics Department, Royal Institute of Technology, Stockholm, Sweden

${ }^{152}$ Departments of Physics and Astronomy, Stony Brook University, Stony Brook, New York, USA

${ }^{153}$ Department of Physics and Astronomy, University of Sussex, Brighton, United Kingdom

${ }^{154}$ School of Physics, University of Sydney, Sydney, Australia

${ }^{155}$ Institute of Physics, Academia Sinica, Taipei, Taiwan

${ }^{156 a}$ E. Andronikashvili Institute of Physics, Iv. Javakhishvili Tbilisi State University, Tbilisi, Georgia

${ }^{156 \mathrm{~b}}$ High Energy Physics Institute, Tbilisi State University, Tbilisi, Georgia

${ }^{157}$ Department of Physics, Technion, Israel Institute of Technology, Haifa, Israel

${ }^{158}$ Raymond and Beverly Sackler School of Physics and Astronomy, Tel Aviv University, Tel Aviv, Israel

${ }^{159}$ Department of Physics, Aristotle University of Thessaloniki, Thessaloniki, Greece

${ }^{160}$ International Center for Elementary Particle Physics and Department of Physics, University of Tokyo, Tokyo, Japan

${ }^{161}$ Graduate School of Science and Technology, Tokyo Metropolitan University, Tokyo, Japan

${ }^{162}$ Department of Physics, Tokyo Institute of Technology, Tokyo, Japan

${ }^{163}$ Tomsk State University, Tomsk, Russia

${ }^{164}$ Department of Physics, University of Toronto, Toronto, Ontario, Canada

${ }^{165 a}$ TRIUMF, Vancouver, British Columbia, Canada

${ }^{165 \mathrm{~b}}$ Department of Physics and Astronomy, York University, Toronto, Ontario, Canada

${ }^{166}$ Division of Physics and Tomonaga Center for the History of the Universe, Faculty of Pure and Applied Sciences, University of Tsukuba, Tsukuba, Japan

${ }^{167}$ Department of Physics and Astronomy, Tufts University, Medford, Massachusetts, USA

${ }^{168}$ Department of Physics and Astronomy, University of California Irvine, Irvine, California, USA

${ }^{169}$ Department of Physics and Astronomy, University of Uppsala, Uppsala, Sweden

${ }^{170}$ Department of Physics, University of Illinois, Urbana, Illinois, USA

${ }^{171}$ Instituto de Física Corpuscular (IFIC), Centro Mixto Universidad de Valencia-CSIC, Valencia, Spain

${ }^{172}$ Department of Physics, University of British Columbia, Vancouver, British Columbia, Canada

${ }^{173}$ Department of Physics and Astronomy, University of Victoria, Victoria, British Columbia, Canada

${ }^{174}$ Fakultät für Physik und Astronomie, Julius-Maximilians-Universität Würzburg, Würzburg, Germany

${ }^{175}$ Department of Physics, University of Warwick, Coventry, United Kingdom

${ }^{176}$ Waseda University, Tokyo, Japan

${ }^{177}$ Department of Particle Physics, Weizmann Institute of Science, Rehovot, Israel 
${ }^{178}$ Department of Physics, University of Wisconsin, Madison, Wisconsin, USA

${ }^{179}$ Fakultät für Mathematik und Naturwissenschaften, Fachgruppe Physik, Bergische Universität Wuppertal, Wuppertal, Germany

${ }^{180}$ Department of Physics, Yale University, New Haven, Connecticut, USA

${ }^{181}$ Yerevan Physics Institute, Yerevan, Armenia

${ }^{a}$ Deceased.

${ }^{\mathrm{b}}$ Also at Department of Physics, King's College London, London, United Kingdom.

${ }^{c}$ Also at Istanbul University, Department of Physics, Istanbul, Turkey.

dAlso at Instituto de Física Teórica de la Universidad Autónoma de Madrid, Spain.

eAlso at Institute of Physics, Azerbaijan Academy of Sciences, Baku, Azerbaijan.

${ }^{\mathrm{f}}$ Also at TRIUMF, Vancouver, British Columbia, Canada.

${ }^{g}$ Also at Department of Physics and Astronomy, University of Louisville, Louisville, Kentucky, USA.

${ }^{\mathrm{h}}$ Also at Department of Physics, California State University, Fresno, California, USA.

${ }^{\mathrm{i}}$ Also at Department of Physics, University of Fribourg, Fribourg, Switzerland.

${ }^{j}$ Also at Departament de Fisica de la Universitat Autonoma de Barcelona, Barcelona, Spain.

${ }^{k}$ Also at Tomsk State University, Tomsk, and Moscow Institute of Physics and Technology State University, Dolgoprudny, Russia.

${ }^{1}$ Also at The Collaborative Innovation Center of Quantum Matter (CICQM), Beijing, China.

${ }^{\mathrm{m}}$ Also at Departamento de Física, Instituto Superior Técnico, Universidade de Lisboa, Lisboa, Portugal.

${ }^{\mathrm{n}}$ Also at Universita di Napoli Parthenope, Napoli, Italy.

${ }^{\circ}$ Also at Institute of Particle Physics (IPP), Canada.

${ }^{\mathrm{p}}$ Also at II. Physikalisches Institut, Georg-August-Universität Göttingen, Göttingen, Germany.

${ }^{\mathrm{q}}$ Also at Horia Hulubei National Institute of Physics and Nuclear Engineering, Bucharest, Romania.

${ }^{\mathrm{r}}$ Also at CPPM, Aix-Marseille Université, CNRS/IN2P3, Marseille, France.

${ }^{\mathrm{s}}$ Also at Department of Physics, St. Petersburg State Polytechnical University, St. Petersburg, Russia.

${ }^{t}$ Also at Borough of Manhattan Community College, City University of New York, New York, USA.

"Also at Department of Financial and Management Engineering, University of the Aegean, Chios, Greece.

${ }^{v}$ Also at Centre for High Performance Computing, CSIR Campus, Rosebank, Cape Town, South Africa.

${ }^{\mathrm{w}}$ Also at Louisiana Tech University, Ruston, Louisiana, USA.

${ }^{\mathrm{x}}$ Also at California State University, East Bay, USA.

${ }^{y}$ Also at Institucio Catalana de Recerca i Estudis Avancats, ICREA, Barcelona, Spain.

${ }^{z}$ Also at Department of Physics, University of Michigan, Ann Arbor, Michigan, USA.

${ }^{a a}$ Also at LAL, Université Paris-Sud, CNRS/IN2P3, Université Paris-Saclay, Orsay, France.

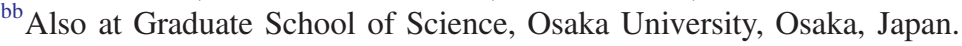

${ }^{\mathrm{cc}}$ Also at Physikalisches Institut, Albert-Ludwigs-Universität Freiburg, Freiburg, Germany.

${ }^{\mathrm{dd}}$ Also at Institute for Mathematics, Astrophysics and Particle Physics, Radboud University Nijmegen/Nikhef, Nijmegen, Netherlands.

${ }^{e e}$ Also at Institute of Theoretical Physics, Ilia State University, Tbilisi, Georgia.

${ }^{\mathrm{ff}}$ Also at CERN, Geneva, Switzerland.

${ }^{g g}$ Also at Department of Physics, Stanford University, USA.

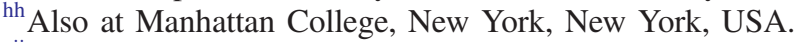

${ }^{i i}$ Also at Joint Institute for Nuclear Research, Dubna, Russia.

${ }^{\mathrm{jj}}$ Also at Hellenic Open University, Patras, Greece.

${ }^{\mathrm{kk}}$ Also at LPNHE, Sorbonne Université, Paris Diderot Sorbonne Paris Cité, CNRS/IN2P3, Paris, France.

${ }^{11}$ Also at The City College of New York, New York, New York, USA.

${ }^{\mathrm{mm}}$ Also at Departamento de Física Teorica y del Cosmos, Universidad de Granada, Granada (Spain), Spain.

${ }^{\mathrm{nn}}$ Also at Department of Physics, California State University, Sacramento, California, USA.

${ }^{\circ}$ Also at Moscow Institute of Physics and Technology State University, Dolgoprudny, Russia.

${ }^{\mathrm{pp}}$ Also at Département de Physique Nucléaire et Corpusculaire, Université de Genève, Genève, Switzerland.

${ }^{\mathrm{qq}}$ Also at Department of Physics and Astronomy, University of Sheffield, Sheffield, United Kingdom.

${ }^{\text {rr }}$ Also at School of Physics, Sun Yat-sen University, Guangzhou, China.

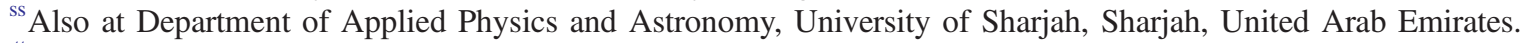

"Also at Institut für Experimentalphysik, Universität Hamburg, Hamburg, Germany.

${ }^{\text {uu }}$ Also at National Research Nuclear University MEPhI, Moscow, Russia.

${ }^{\mathrm{vv}}$ Also at Institute for Particle and Nuclear Physics, Wigner Research Centre for Physics, Budapest, Hungary.

${ }^{w w}$ Also at Giresun University, Faculty of Engineering, Giresun, Turkey.

${ }^{x x}$ Also at Institute of Physics, Academia Sinica, Taipei, Taiwan. 